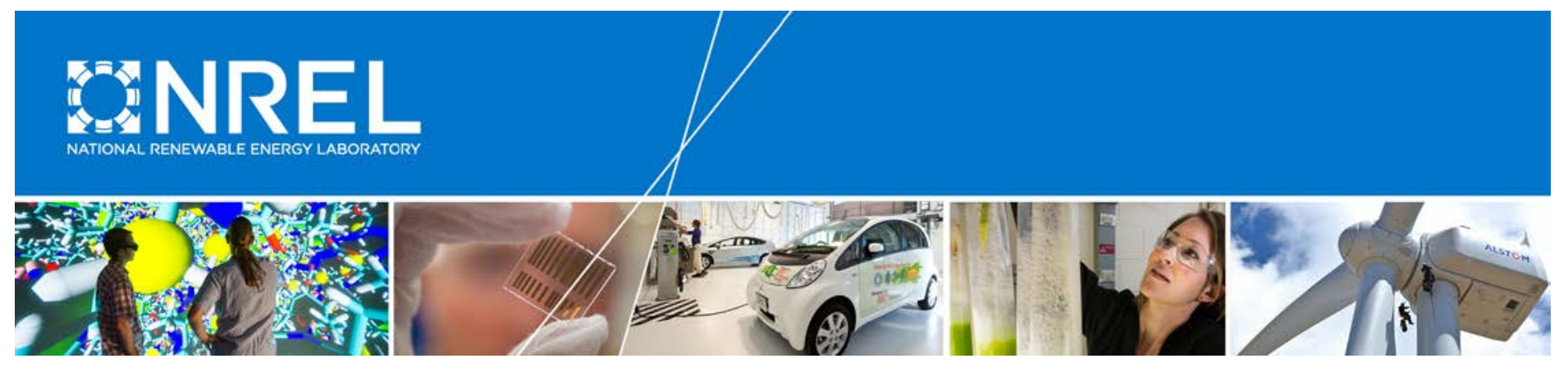

\title{
An Analytical Formulation for Sizing and Estimating the Dimensions and Weight of Wind Turbine Hub and Drivetrain Components
}

Y. Guo, T. Parsons, R. King, K. Dykes, and $P$. Veers National Renewable Energy Laboratory

NREL is a national laboratory of the U.S. Department of Energy Office of Energy Efficiency \& Renewable Energy Operated by the Alliance for Sustainable Energy, LLC

This report is available at no cost from the National Renewable Energy Laboratory (NREL) at www.nrel.gov/publications.

Technical Report

NREL/TP-5000-63008

June 2015

Contract No. DE-AC36-08GO28308 


\section{An Analytical Formulation for Sizing and Estimating the Dimensions and Weight of Wind Turbine Hub and Drivetrain Components}

Y. Guo, T. Parsons, R. King, K. Dykes, and $P$. Veers

National Renewable Energy Laboratory

Prepared under Task No. WE14.3G01

NREL is a national laboratory of the U.S. Department of Energy Office of Energy Efficiency \& Renewable Energy Operated by the Alliance for Sustainable Energy, LLC

This report is available at no cost from the National Renewable Energy Laboratory (NREL) at www.nrel.gov/publications.

National Renewable Energy Laboratory 15013 Denver West Parkway Golden, CO 80401

303-275-3000 • www.nrel.gov

\section{Technical Report}

NREL/TP-5000-63008

June 2015

Contract No. DE-AC36-08G028308 


\title{
NOTICE
}

This report was prepared as an account of work sponsored by an agency of the United States government. Neither the United States government nor any agency thereof, nor any of their employees, makes any warranty, express or implied, or assumes any legal liability or responsibility for the accuracy, completeness, or usefulness of any information, apparatus, product, or process disclosed, or represents that its use would not infringe privately owned rights. Reference herein to any specific commercial product, process, or service by trade name, trademark, manufacturer, or otherwise does not necessarily constitute or imply its endorsement, recommendation, or favoring by the United States government or any agency thereof. The views and opinions of authors expressed herein do not necessarily state or reflect those of the United States government or any agency thereof.

This report is available at no cost from the National Renewable Energy Laboratory (NREL) at www.nrel.gov/publications.

Available electronically at SciTech Connect http:/www.osti.gov/scitech

Available for a processing fee to U.S. Department of Energy and its contractors, in paper, from:

\author{
U.S. Department of Energy \\ Office of Scientific and Technical Information \\ P.O. Box 62 \\ Oak Ridge, TN 37831-0062 \\ OSTI http://www.osti.gov \\ Phone: 865.576.8401 \\ Fax: 865.576.5728 \\ Email: reports@osti.gov
}

Available for sale to the public, in paper, from:

\author{
U.S. Department of Commerce \\ National Technical Information Service \\ 5301 Shawnee Road \\ Alexandra, VA 22312 \\ NTIS http://www.ntis.gov \\ Phone: 800.553 .6847 or 703.605 .6000 \\ Fax: 703.605.6900 \\ Email: orders@ntis.gov
}




\section{Abstract}

This report summarizes the theory, verification, and validation of a new sizing tool for wind turbine drivetrain components, the Drivetrain Systems Engineering (DriveSE) tool. DriveSE calculates the dimensions and mass properties of the hub, main shaft, main bearing(s), gearbox, bedplate, transformer if up-tower, and yaw system. The level of fidelity for each component varies depending on whether semiempirical parametric or physics-based models are used. The physics-based models have internal iteration schemes based on system constraints and design criteria. Every model is validated against available industry data or finite-element analysis. The verification and validation results show that the models reasonably capture primary drivers for the sizing and design of major drivetrain components. 


\section{List of Terms}

\begin{tabular}{l|l|}
\hline Acronyms & \multicolumn{1}{c|}{ Meaning } \\
\hline \hline AGMA & American Gear Manufacturer's Association \\
CARB & compact-aligning roller bearing \\
CM & center of mass \\
COG & center of gravity \\
CRB & cylindrical roller bearing \\
DriveSE & Drivetrain Systems Engineering \\
eep & planetary-planetary-parallel \\
epp & planetary-parallel-parallel \\
FEA & finite-element analysis \\
GRC & Gearbox Reliability Collaborative \\
IEC & International Electrotechnical Commission \\
ISO & International Organization for Standardization \\
HSS & high-speed shaft \\
RB & single-row deep-groove radial ball bearing \\
RNA & rotor-nacelle assembly \\
SRB & spherical roller bearing \\
TRB1 & single-row tapered roller bearing \\
TRB2 & double-row tapered roller bearing \\
\hline \hline
\end{tabular}

\begin{tabular}{lll}
\hline Nomenclature & \\
\hline \hline$A_{w}$ & Weibull scale parameter \\
$B$ & Blade number \\
$B_{p}$ & Number of planet gears \\
$C_{L}$ & Coefficient of lift \\
$C M$ & Center of mass \\
$d$ & Diameter \\
$d_{p}$ & Gear diameter \\
$D$ & Damage caused by fatigue \\
$E$ & Young's modulus \\
$f$ & Frequency \\
$F$ & Force or load \\
$F W$ & Face width \\
$F \Delta^{*}()$ & Stochastic load range at given load count \\
$H$ & Height \\
$I$ & Second moment of area \\
$I_{t}$ & Turbulence intensity factor \\
$k$ & Safety factor \\
$K_{A G}$ & Application factor \\
$K_{r}$ & Scaling factor \\
$K_{s h}$ & Shaft factor \\
$K_{v}$ & Dynamic factor \\
$k_{w}$ & Weibull shape parameter \\
$K_{\gamma b}$ & Load-sharing factor between rows \\
$K_{\gamma p}$ & Load-sharing factor among planets \\
$L$ & Length \\
\hline & \\
\hline
\end{tabular}

iv 


\begin{tabular}{|c|c|}
\hline \multicolumn{2}{|r|}{ - continued from previous page } \\
\hline Nomenclature & Meaning \\
\hline$m$ & Mass \\
\hline$M$ & Moment \\
\hline$n(), N()$ & Number of stress cycles, number of cycles to failure at given amplitude \\
\hline$N_{f}$ & Maximum number of load cycles experienced by components \\
\hline$n_{p}$ & Speed \\
\hline$p$ & Diametral pitch \\
\hline$P$ & Power \\
\hline$p_{0}$ & Aerodynamic line-load \\
\hline$Q_{o}$ & Input torque to the main shaft \\
\hline$Q_{p}$ & Input torque to the pinion \\
\hline$r$ & Radius \\
\hline$S_{f}$ & Fatigue strength \\
\hline$S_{F}$ & Stress range caused by specified force (fatigue) \\
\hline$S_{M}$ & Stress range caused by specified moment (fatigue) \\
\hline$S_{y}$ & Yielding strength \\
\hline$S_{m}$ & Fatigue failure point at $10^{3}$ cycles \\
\hline$S_{U T}$ & Ultimate material strength \\
\hline$t$ & Time \\
\hline$t h$ & Thickness \\
\hline$T$ & Torque \\
\hline$T D$ & Tip deflection \\
\hline$T_{L}$ & Turbine life \\
\hline$U$ & Speed ratio \\
\hline$v$ & Transverse deflection \\
\hline$V$ & Volume \\
\hline$V_{\min }, V_{\max }$ & Cut-in, cut-out windspeeds \\
\hline$V_{0}$ & Nominal wind speed \\
\hline$W$ & Weight \\
\hline$W_{G B P N}$ & Gear pair weight \\
\hline$W_{t}$ & Tangential driving force \\
\hline$X$ & Tip-speed ratio \\
\hline$\eta_{d}$ & Drivetrain efficiency \\
\hline$\gamma$ & Bedplate tilt angle \\
\hline$\omega$ & Weight per unit length \\
\hline$\sigma$ & Normal stress \\
\hline$\tau$ & Shear stress \\
\hline$\sigma_{v}$ & von Mises stress \\
\hline$\sigma_{1}$ & Maximum principal stress \\
\hline$\sigma_{a}$ & Alternating stress \\
\hline$\sigma_{a}$ & Mean stress \\
\hline$\sigma_{e}$ & Effective alternating stress at failure \\
\hline$\rho$ & Density \\
\hline Subscript & Meaning \\
\hline as & Main bearing-main shaft center of mass \\
\hline$a x$ & Axial \\
\hline$b$ & Blade \\
\hline bend & Bending stress range \\
\hline$b g$ & Main bearing-gearbox trunnion \\
\hline$c$ & Characteristic \\
\hline$e q$ & Equivalent stress range \\
\hline$f^{1}$ & Main shaft flange \\
\hline$g b$ & Bearing-gear coupling \\
\hline & Continued on next page \\
\hline
\end{tabular}




\begin{tabular}{|l|l|}
\multicolumn{2}{c}{ - continued from previous page } \\
\hline \multicolumn{1}{|c}{ Nomenclature } & \multicolumn{1}{c|}{ Meaning } \\
$g b x$ & Gearbox \\
$g c$ & Gearbox \\
$G E$ & Gear coupling \\
$g e n$ & Generator \\
$h b$ & Generator \\
$h s$ & Hub \\
$m b 1, m b 2$ & High-speed shaft/coupling \\
$m b$ & Upwind and downwind main bearing for four-point suspension \\
$m s$ & Main bearing for three-point suspension \\
$m s, i$ & Main shaft \\
$n o r m$ & Inner main shaft \\
$p$ & Normal stress range \\
$r$ & Planet \\
$r a$ & Rotor \\
$r b$ & Radial \\
$R N A$ & Rotor-main bearing \\
$s$ & Rotor-nacelle assembly \\
$r g$ & Sun \\
$t$ & Ring \\
$T F$ & Transformer \\
$T W$ & Transformer \\
\hline \hline Superscript & Tower \\
\hline \hline$x, y, z$ & Meaning \\
$d e, m e a n, u l t, m a x$ & Load type (deterministic, mean, ultimate, maximum) \\
$s t$ & stochastic \\
\hline \hline & \\
\hline
\end{tabular}




\section{Table of Contents}

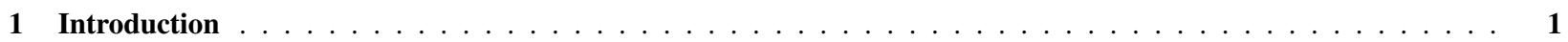

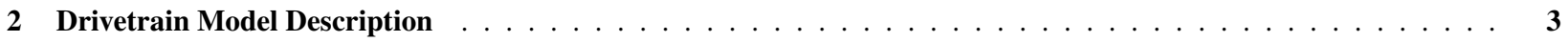

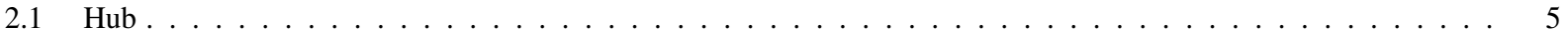

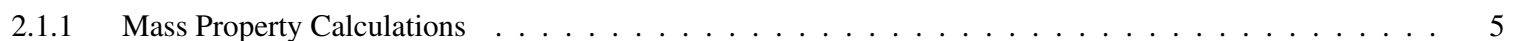

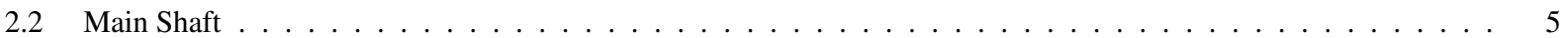

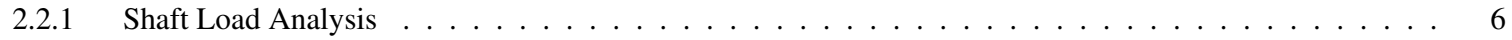

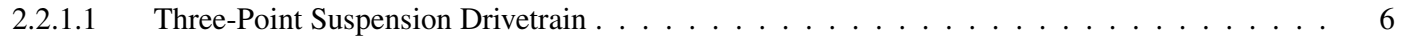

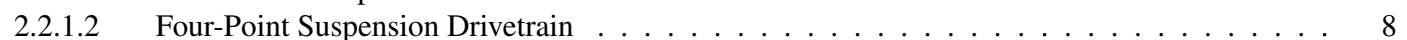

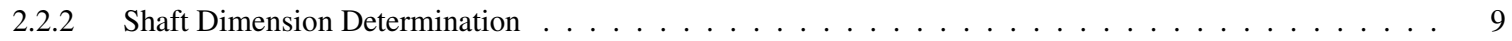

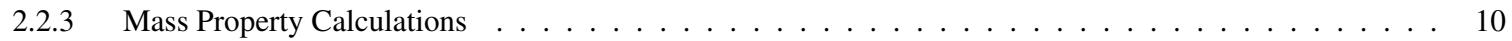

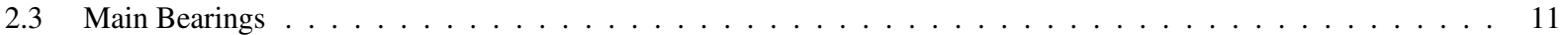

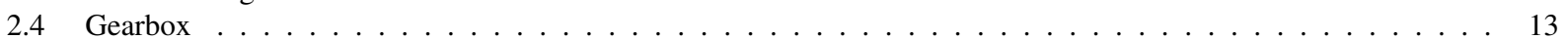

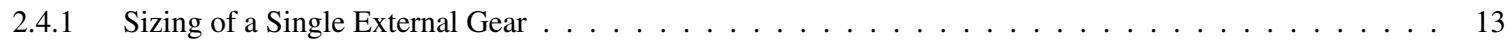

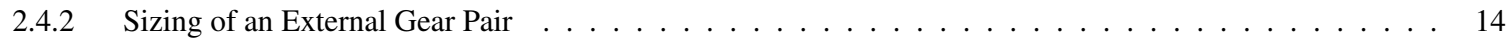

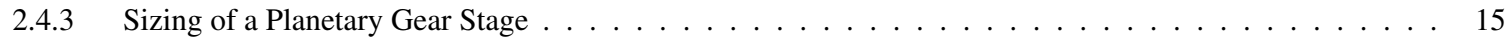

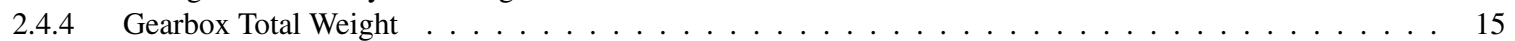

2.4.5 Gearbox Speed Ratio Optimization . . . . . . . . . . . . . . . . . . . . . . . 15

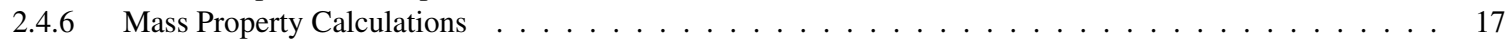

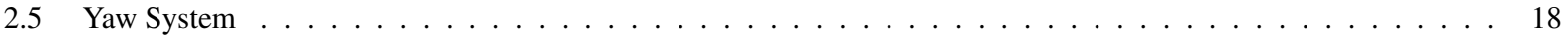

2.5.1 Mass Property Calculations _. . . . . . . . . . . . . . . . . . . . . . . . . . . . 19

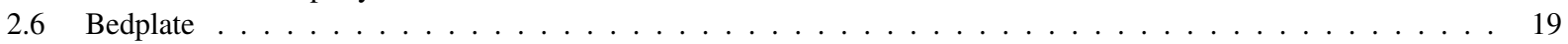

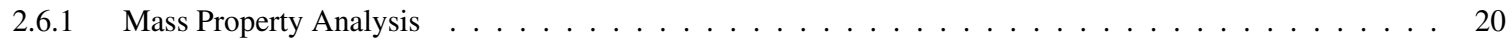

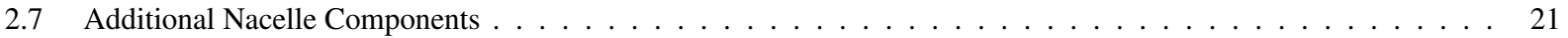

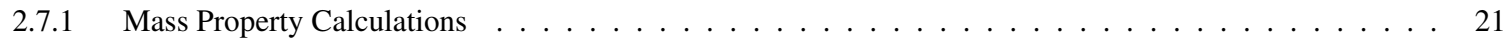

3 Model Verification Against Higher Fidelity Models $\ldots \ldots \ldots \ldots$

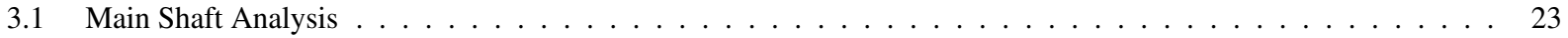

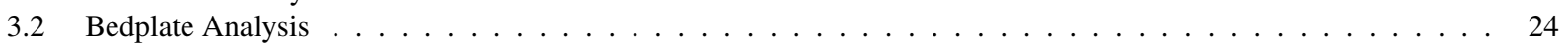

4 Model Validation and Results $\ldots \ldots \ldots \ldots \ldots \ldots$

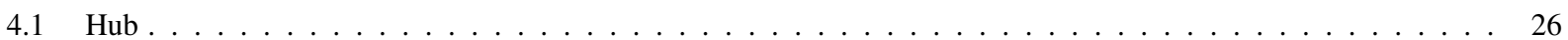

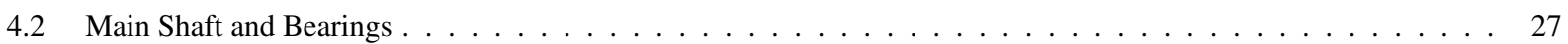

4.3 Gearbox Stage Ratio and Weight . . . . . . . . . . . . . . . . . . . . . . . . 28

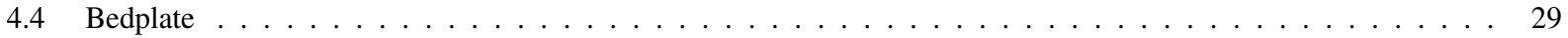

4.5 Nacelle Mass . . . . . . . . . . . . . . . . . . . . . . . . . . 30

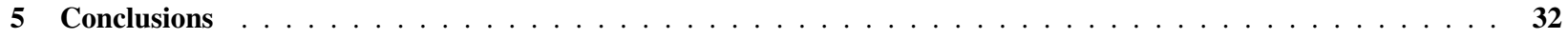

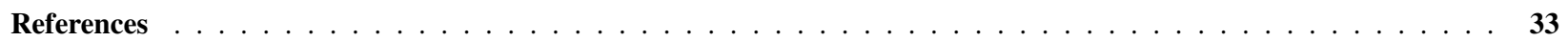

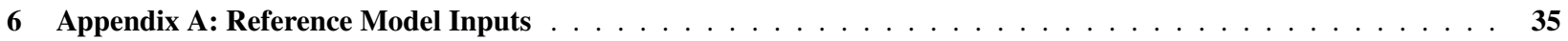

7 Appendix B: Shaft and Bearing Design with Parameterized Fatigue Spectrum (Optional) $\ldots \ldots \ldots$

8 Appendix C: Parameterized Fatigue Loads Definition $\ldots \ldots \ldots \ldots$

9 Appendix D: Simplified Four-Point Suspension Main Shaft Model (Optional) . . . . . . . . . . . . . . . . . . . 49

vii 


\section{List of Figures}

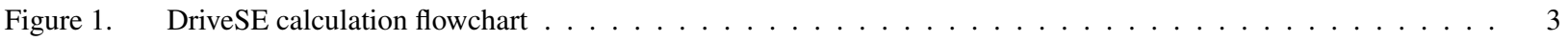

Figure 2. Force diagram of a main shaft in a three-point suspension drivetrain . . . . . . . . . . . . . 6

Figure 3. Force diagram of a main shaft in a four-point suspension drivetrain . . . . . . . . . . . . . . 8

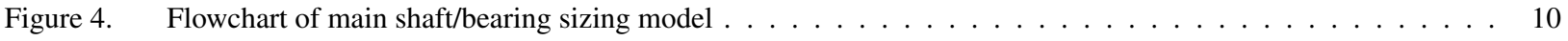

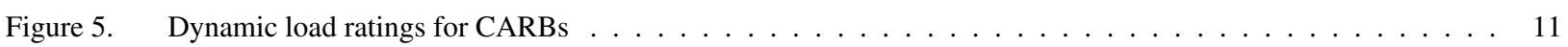

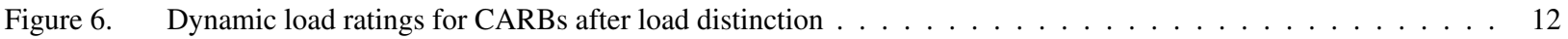

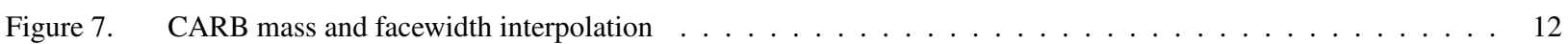

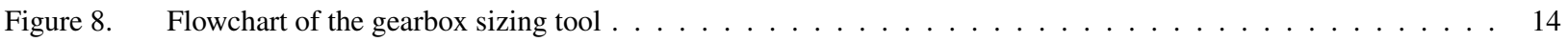

Figure 9. Flowchart of the gearbox iteration process for determining the stage ratios . . . . . . . . . . . . . . 18

Figure 10. (A) Loads and constraints applied to the 750-kW LSS; (B) the mesh used in the analysis; and the plots of (C)

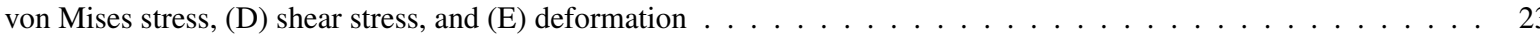

Figure 11. DriveSE basic I-beam assembly with large front cast iron beam and long rear steel piece. Note the split lines

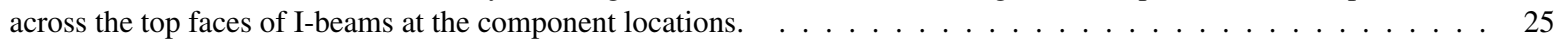

Figure 12. Industry trend and model results: hub mass compared to turbine nameplate rating . . . . . . . . . . . 26

Figure 13. Industry trend and model results: main shaft mass compared to turbine rating . . . . . . . . . . . . . . 28

Figure 14. Industry trend and model results: gearbox weight compared to rated torque . . . . . . . . . . . . . . 29

Figure 15. Industry trend and model results: bedplate mass compared to rotor diameter . . . . . . . . . . . . . . . . 30

Figure 16. Industry trend and model results: nacelle mass compared to rotor diameter . . . . . . . . . . . 30

Figure 17. Flowchart of main shaft and bearing fatigue sizing tool . . . . . . . . . . . . . . . . . . . . . 39

Figure 18. Force and moment spectra defined by DS472 using inputs from a 750-kW rotor . . . . . . . . . . . . . . 47

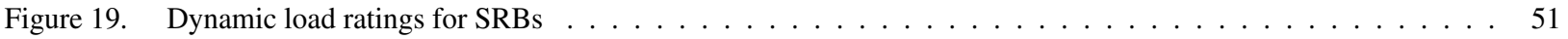

Figure 20. SRB mass and facewidth interpolation $\ldots \ldots \ldots \ldots$

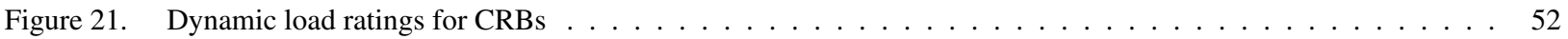

Figure 22. $\quad$ CRB mass and facewidth interpolation $\ldots \ldots \ldots \ldots$

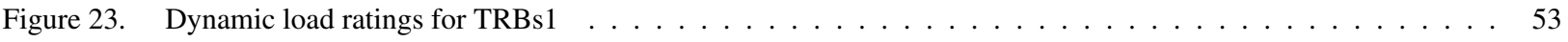

Figure 24. TRB1 mass and facewidth interpolation . . . . . . . . . . . . . . . . . . . . 53

Figure 25. Dynamic load ratings for TRBs $\ldots \ldots \ldots \ldots \ldots$

Figure 26. TRB2 mass and facewidth interpolation . . . . . . . . . . . . . . . . . . . . . . . 54

Figure 27. Dynamic load ratings for $\mathrm{RBs} \ldots \ldots \ldots \ldots$

Figure $28 . \quad$ RB mass and facewidth interpolation $\ldots \ldots \ldots \ldots$

viii 


\section{List of Tables}

Table 1. Required Parameters for DriveSE Component Sizing . . . . . . . . . . . . . . . . . 4

Table 2. Maximum Ranges for Slopes and Transverse Deflections (Shigley et al. 2003) . . . . . . . . . . . . . . 6

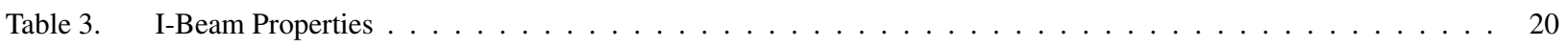

Table 4. Loads Applied to Main Shaft Components, in Global Nacelle Coordinate System . . . . . . . . . . . . 24

Table 5. Resulting Maximum Stress Concentrations and Deflections at the Location of the Bearings . . . . . . . . . . 24

Table 6. Bending Stress and Tip Deflection Compared for Rear Beams . . . . . . . . . . . . . . . . . . . . 24

Table 7. Maximum Bending Stress and Tip Deflection Compared for Front Beams . . . . . . . . . . . . . . . 25

Table 8. Comparison of Hub Model Outputs to FE Model Masses . . . . . . . . . . . . . . . . . 26

Table 9. Comparison of Nonfatigue Main Shaft Model Output and Solid Mass Dimensions . . . . . . . . . . . . . . 27

Table 10. Comparison of Fatigue Main Shaft Model Output and FE Model Dimensions ～. . . . . . . . . . . . . . . . . 27

Table 11. Comparison of Fatigue and Nonfatigue Models to the FE Model Mass . . . . . . . . . . . . . . . . 27

Table 12. Gearbox Speed Ratio per Stage of the GRC 750-kW Gearbox . . . . . . . . . . . . . . . . . . 28

Table 13. Comparison of Bedplate Model Outputs to FE Model Masses . . . . . . . . . . . . . . . . . . . . . . . . 29

Table 14. Effects of Transformer Location on Bedplate Dimensions and Mass for the 1.5-MW Turbine . . . . . . . . . 29

Table 15. $750-\mathrm{kW}$ Reference Inputs . . . . . . . . . . . . . . . . . . . . . . . . 35

Table 16. $750-\mathrm{kW}$ Reference Fatigue Inputs . . . . . . . . . . . . . . . . . . . . . . . . . . 35

Table 17. 1.5-MW Reference Inputs . . . . . . . . . . . . . . . . . . . . . . . 36

Table 18. 1.5-MW Reference Fatigue Inputs . . . . . . . . . . . . . . . . . . . . . . . . 36

Table 19. $5-\mathrm{MW}$ Reference Inputs . . . . . . . . . . . . . . . . . . . . . . . . 37

Table 20. 5 -MW Reference Fatigue Inputs . . . . . . . . . . . . . . . . . . . . . . . 37

Table 21. Additional Inputs Required for Parameterized Fatigue Analysis . . . . . . . . . . . . . . . . . . . 38

Table 22. Bearing Calculation Factors Used by Bearing Type . . . . . . . . . . . . . . . . . . 44

Table 23. Description of Three Stress Sources Considered in the Main Shaft Fatigue Analysis . . . . . . . . . . . . . 45

Table 24. Relationship Between IEC Class and Turbulence Intensity Factor . . . . . . . . . . . . . . . . . . . . 47 


\section{Introduction}

Wind turbine drivetrains serve the fundamental role of converting the aerodynamic torque of a rotor into electrical power that can be fed to the connecting grid. They also serve as the load path for transferring all of the nontorque loads from the rotor to the tower, which then transfers those loads to the ground. Accordingly, the drivetrain design affects and is affected by the rest of the wind turbine design.

Typical design practice for a wind turbine considers constraints from the drivetrain on the rotor and tower design and vice versa. However, improvements to overall wind turbine system design can be obtained by an integrated approach that considers the simultaneous and integrated design of the rotor, drivetrain, and tower (Ning and Dykes 2014). There are important trade-offs in the design of these subsystems that affect each other and if not considered together, the overall system cost can be higher. This was recently demonstrated in a study of the effect of tip-speed constraints on system design and cost in which an improved rotor design for lower weight resulted in large aerodynamics loads on the drivetrain that resulted in a net increase in system cost (Dykes et al. 2014a). Allowing system cost and performance objectives to dictate the design of the wind turbine subsystems will result in a truly optimal overall wind turbine design.

To enable this system-level optimization, physics-based models of all major system components are required to explicitly capture the trade-offs between the design of different components. A previously developed physical sizing model for the drivetrain, known as the Sunderland model, was established by the University of Sunderland in the late 1990s (Harrison and Jenkins 1993). The Sunderland model used semiempirical formulations to calculate the masses of major wind turbine components. These formulations were derived from a limited industry database that does not represent today's fleet. Moreover, the Sunderland model cannot be used for design optimizations of wind turbine components. Variations of industry designs and challenges in extrapolating the limited industry data decrease the design accuracy of wind turbine drivetrains.

The Drivetrain Systems Engineering (DriveSE) model was developed by the National Renewable Energy Laboratory (NREL) in 2014 using a direct physics-based approach than the semiempirical approach used in the Sunderland model. The physics-based approach has advantages for turbine-level system optimization because it considers a wider variety of drivetrain configuration, is more independent of industry data for calibration, and can be applied when designing new turbines that are on a larger scale than those that exist today. In addition, the model is made publicly available and is open source for adaptation to additional configurations. DriveSE can be used for standalone drivetrain analysis and design or as part of a larger wind turbine or wind plant study. Two case studies using DriveSE investigated the effects of rotor configurations on the drivetrain and the rest of machine (Dykes et al. 2014a) (Ning and Dykes 2014).

DriveSE uses a rigorous set of physics-based models to estimate the size of a subset of the major load-bearing components (the main shaft, main bearing(s), gearbox, and bedplate) and parametric formulations representative of current wind turbine technology for the remaining components (the hub and yaw system). The high-speed side of the drivetrain, which includes the high-speed coupling, mechanical brake, generator, and other auxiliary components, are modeled semiempirically (Harrison and Jenkins 1993) (DriveWPACT 2013). An up-tower transformer model based on industry data is also included. DriveSE considers the aerodynamic loads and rotor properties and calculates the mass properties and dimensions of major drivetrain components; the overall nacelle properties can then be used in subsequent tower design and analysis or as part of a system-level optimization of the wind turbine. In addition, the resulting mass and size estimates can then be fed into a turbine capital-cost model as well as a balance-of-station cost model that considers the cost of assembly and installation of a wind turbine so that a full cost analysis of a wind power plant at the system level can be performed. Consequently, although DriveSE can be used for drivetrain-specific analysis (King et al. 2014), the model set can also be used as part of larger wind turbine and plant studies.

Geared drivetrains, the most prevalent design for land-based wind turbines, consist of a main shaft, main bearing(s), gearbox, generator coupling, and generator. Various manufacturers use different rotor supports and bearing configurations, which can be grouped into four categories: (1) three-point suspension; (2) two-main-bearing suspension (four-point suspension); (3) integrated drivetrain; and (4) hub-supported drivetrain. In the three-point suspension, the rear main bearing is integrated into the gearbox at the planetary stage as the planetary carrier bearing and together they support the non-torque loads. The two-main-bearing suspension uses two separate main bearings that carry the majority of the nontorque loads from the rotor and transmit them into the tower through the bedplate. The integrated drivetrain integrates the main bearings into the gearbox. The nontorque loads are transmitted through the gearbox housing. The hub-supported drivetrain uses flexible couplings to connect the rotor with the main shaft and thus isolates any nontorque loads from the drivetrain. Among all different drivetrain configurations, the three-point suspension and two-main-bearing suspension (four-point suspension) are most common; as a result, these are the configurations modeled in DriveSE. Low-speed and direct-drive generators are outside the scope of the work presented here and will be addressed in future model versions. The gearbox model of DriveSE includes several layouts of parallel and planetary

1 
gear stages. The constraints on the system design are as close as possible to those used in practice for designing commercial components.

The resulting drivetrain designs are compared to actual industry data or higher fidelity finite-element analysis (FEA). For the physics-based models, verification is performed against higher fidelity finite-element models for key design criteria. In this work, this involved creating representations of each component for different sizes using data on real turbine components when possible. An iterative process was used to evaluate the DriveSE models compared to the higher fidelity models and corrections to the DriveSE models were made as necessary. For both the physics-based and parametric models, validation of each model was performed against available industry data. The advantages and limitations of each model are identified to inform the use of the models in subsequent analysis efforts. 


\section{Drivetrain Model Description}

DriveSE consists of a series of coupled mathematical models for drivetrain components, as shown in Figure 1. It includes the hub, main shaft, main bearings, gearbox, bedplate, transformer if up-tower, and yaw system. The remainder of the components in the hub and nacelle systems are sized using DriveWPACT (DriveWPACT 2013) that is based on empirical data. The master routine, DriveWPACT, interfaces with other wind turbine components, specifically, the rotor and tower. At this top level, design criteria on allowable stress and deflection are inherently included for individual drivetrain components. These design criteria, together with the minimum weight objective, are used for sizing drivetrain components.

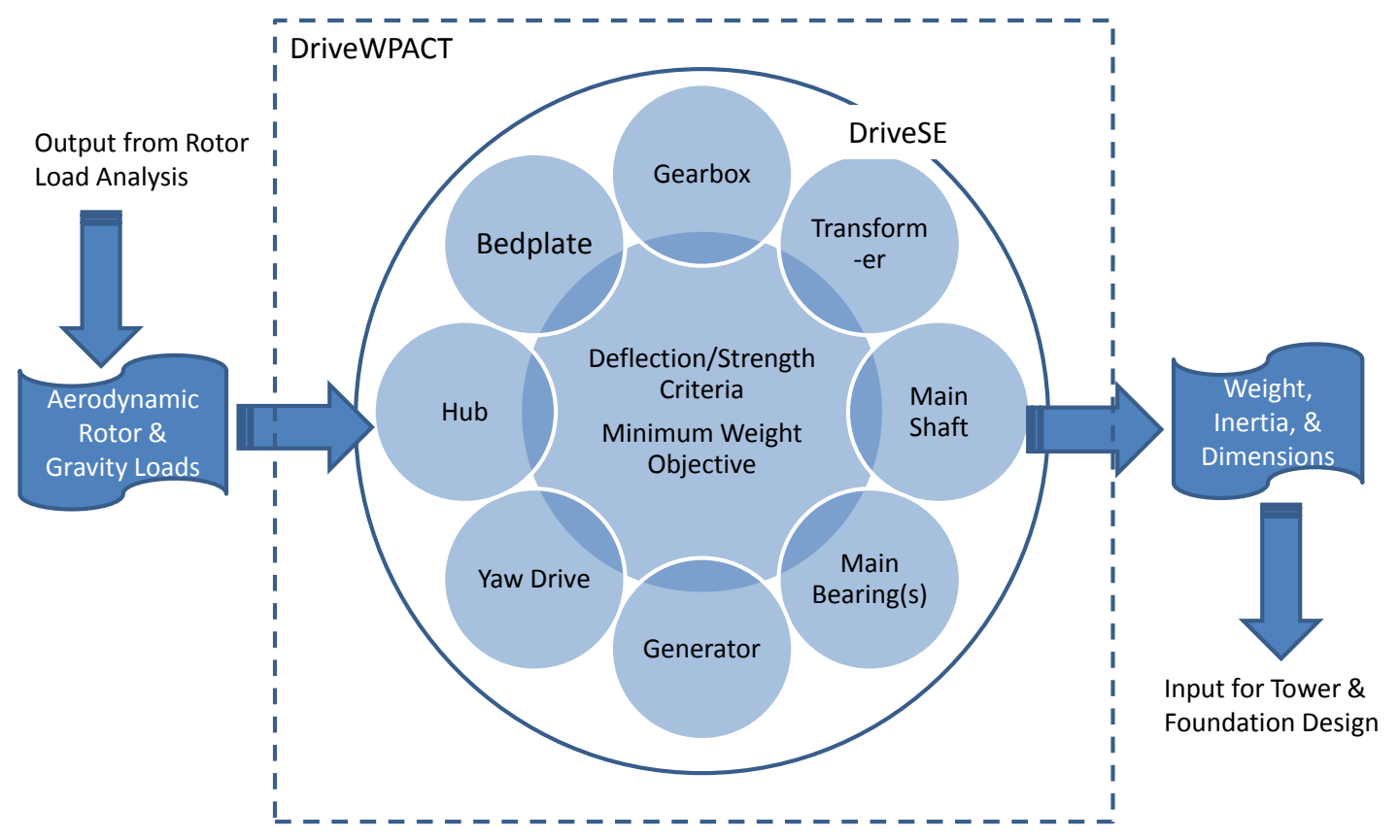

Figure 1. DriveSE calculation flowchart

A number of assumptions have been made to facilitate the drivetrain model development. The current effort focuses on component designs driven by maximum stress analyses and deflection criteria. By using industry standard safety margins, this approach sizes components to avoid catastrophic failure. The deflection constraints ensure that components are properly aligned and within geometric limits for bearing alignment and gear tooth meshing, among others. These geometric requirements are an important part of the integrated design work flow connecting the main bearings, main shaft, and gearbox components. The optional fatigue analyses for the main shaft and bearings are based on a linear damage assumption across the design life of a turbine. Another general assumption includes homogeneously distributed component masses for calculating the moment of inertia and center of mass $(\mathrm{CM})$. When models rely on scaling arguments, such as for the hub or transformer mass in sizing the bedplate, the departure from physics-based analysis is noted.

Major model inputs include the aerodynamic rotor loads, gravity loads, gearbox configuration parameters, and overall turbine design parameters such as rotor overhang and gearbox location. The design loads used in the model are based on those that would be obtained via wind turbine dynamic simulations, such as those from an aeroelastic code or similar model. The required input loads and other key inputs for each drivetrain component are summarized in Table 1. External loads are specified using the load analysis results; the internal loads are calculated by DriveSE and drive the design of the components downstream. Examples of model inputs that define each reference turbine are included in Appendix A.

The outputs of DriveSE fall into two categories: component outputs and system outputs. Component outputs include the dimensions and mass properties that are preliminary design parameters for drivetrain components. The current model implementation calculates the dimension, mass, CM, and moment of inertia for the hub, main shaft, upwind main bearing, downwind main bear- 
Table 1. Required Parameters for DriveSE Component Sizing

\begin{tabular}{lcccccc}
\hline Input Parameter & Hub & Main Shaft & Main Bearing & Gearbox & Bedplate & Yaw \\
\hline Power Rating (External) & $\times$ & $\times$ & $\times$ & $\times$ & - & - \\
Rotor Diameter (External) & - & - & - & - & - & $\times$ \\
Blade Root Diameter (External) & $\times$ & - & - & - & - & - \\
Drivetrain Topology (External) & - & $\times$ & $\times$ & $\times$ & $\times$ & - \\
Rotor Weight (External) & - & $\times$ & $\times$ & - & $\times$ & - \\
Rotor Overhang (External) & - & $\times$ & $\times$ & - & $\times$ & - \\
Rotor Loads (External) & - & $\times$ & $\times$ & - & - & - \\
Rotor Torque (External) & - & $\times$ & $\times$ & $\times$ & - & - \\
Gearbox Ratio (External) & - & - & - & $\times$ & - & - \\
Bearing Type (External) & - & - & $\times$ & - & - & - \\
Gearbox Topology (External) & - & - & - & $\times$ & - & - \\
Generator Type (External) & - & - & - & - & - & - \\
\hline Main Shaft Dimensions (Internal) & - & - & $\times$ & - & $\times$ & - \\
Bearing Weight (Internal) & - & - & - & - & $\times$ & - \\
Gearbox Weight (Internal) & - & - & - & - & $\times$ & - \\
Generator Weight (Internal) & - & - & - & - & $\times$ & - \\
Converter Weight (Internal) & - & - & - & - & $\times$ & - \\
Transformer Weight (Internal) & - & - & - & - & $\times$ & - \\
\hline
\end{tabular}

ing (if used), gearbox, bedplate, generator, and yaw system. For the gearbox, gear ratios per stage, volumes, and masses that can be used for gearbox internal component design are also provided. The system outputs are the cumulative weight, moments of inertia, and center of gravity of the entire hub and nacelle assemblies, which are used as inputs at the tower design level and also for wind turbine and plant cost models.

Each component model uses a unique design approach. The hub is modeled of scaling arguments calibrated to industry data. The current implementation treats the hub as a thin-walled ductile cast iron cylinder with circular holes for blade root openings and low-speed shaft flange. The hub outer dimensions and thickness scale to the rotor diameter and blade root thickness.

The main shaft and bearing design uses a physics-based model. It is sized first by determining the length from deflection limitations imposed by main bearings, with a maximum length constrained by the rotor overhang distance. Distortion energy failure theory is used to determine the outer diameters at the main bearings, with the final design consisting of a hollow shaft with a taper or cylinder between bearing locations. The shaft shape is automatically determined by DriveSE. Main bearings are then selected based on shaft geometry and load capacity from the DriveSE database. Finally, the shaft dimensions are updated to match the closest bearing bore diameters.

Gearboxes are one of the most expensive components in wind turbine drivetrains and being able to estimate their weight accurately is important for calculating overall drivetrain capital, operational, and maintenance costs. DriveSE designs the gearbox for the minimum weight by optimizing the speed ratio of each stage. The model requires a minimum input set that includes torque, overall speed ratio, number of stages, and configuration. DriveSE considers gearboxes with two or three stages with different combinations of planetary and parallel stages. The gearbox model outputs the weight, volume, and speed ratio of each gearbox stage as well as the overall gearbox weight. Detailed dimensions of gearbox internal components are not provided at this time.

The bedplate size is approximated by modeling the bedplate as two parallel I-beams and separately treating the upwind and downwind sections. The upwind section is assumed to be made of ductile cast iron; the downwind section is steel. Weights of the drivetrain components are applied to the bedplate structure at the CM of each component, and rotor aerodynamic loads are superimposed on the upwind bedplate section. The upwind and downwind bedplate sections are individually sized to meet deflection and bending stress constraints that are automatically defined inside the program.

A friction plate yaw bearing at the nacelle tower and several yaw motors comprise the yaw system. The friction plate bearing is treated as a steel annulus and is sized empirically according to the tower top diameter and rotor diameter. The motors are assumed to be of a common Bonfiglioli design and the number of motors is a function of the rotor diameter if not specified by the user. 
The rest of the drivetrain and hub system are included in the analysis for sizing the bedplate and to compute the overall drivetrain weight, inertia, and CM. Masses and sizes of these components are currently calculated using a modified version of DriveWPACT (DriveWPACT 2013), with updated CM calculations based on component sizes and CM optimization. The DriveWPACT models for the high-speed shaft (HSS), coupling, brake, and generator are highly simplified parametric models. A physics-based generator and generator-coupling model is not included at this stage, although a parametric model of the transformer has been added.

\subsection{Hub}

Because of its geometric complexity, DriveSE models the hub entirely from scaling arguments. It will be refined in future studies. The current implementation treats the hub as a thin-walled, ductile, cast iron cylinder with holes for blade root openings and main shaft flanges. The hub outer dimensions and thickness scale to the blade root thickness. Pitch motors and bearings are not considered at this point and are modeled using DriveWPACT.

The hub radius is assumed to be $1.1 \times$ the blade root radius, the hub height is assumed to be $2.8 \times$ the hub radius, and the hub thickness is assumed to be $1 / 10^{\text {th }}$ the hub radius. These relationships are based from existing hub designs and will be updated as new data become available.

\subsubsection{Mass Property Calculations}

The total hub material volume $V_{h b}$ is then given by:

$$
V_{h b}=2 \pi r_{h b} H_{h b} t h_{h b}-(1+B) \pi r_{h b}^{2} t h_{h b}
$$

where material has been removed for each blade root and the main shaft flange opening is assumed to be the same size as a blade root. The hub mass, $m_{h b}$, is simply calculated by $m_{h b}=\rho V_{h b}$, where $\rho$ is the hub cast iron density, which is $7,200 \mathrm{~kg} / \mathrm{m}^{3}$ in this module.

In case the blade root diameter is unknown to the user, a default is set using the scaling relationship shown in Eq. 2.2. This relationship was found using industry data gathered from a wide range of turbines, and scales much better to the machine's power rating, $P[M W]$, than to its rotor diameter.

$$
d_{b}=2.659 \times P^{0.325}
$$

The $\mathrm{CM}$ of the hub is determined by the location of the upwind main bearing, and the variable $L_{r b}$ (shown in Figure 2) as:

$$
\left[\begin{array}{ll}
C M_{h b}^{x} & =C M_{m b 1}-L_{r b} \\
C M_{h b}^{y} & =0 \\
C M_{h b}^{z} & =C M_{m b 1}
\end{array}\right]
$$

\subsection{Main Shaft}

Important main shaft dimensions include length, outer diameter, inner diameter, and tapered angle. Shaft length is designed to meet deflection limitations imposed by the main bearing(s) mounted on the shaft. It is a key factor in determining the CM of the drivetrain. Shaft diameters are determined using the distortion energy failure theory with a peak load safety factor of 2.5 (AGMA 2008) applied. The main shaft can have a cylindrical or tapered shape, which is determined automatically according to the carried loads at shaft ends. DriveSE also provides optional shaft design with parameterized fatigue spectrum, which is detailed in Appendix B/C.

Shafts are designed to meet the requirements on deflections and rigidity. When shaft preliminary design is complete, the shaft deflections and misalignments are calculated and checked to determine if they meet the deflection requirements at critical locations, including at the interfaces with the main bearing(s) and the low-speed stage of the gearbox. The typical maximum ranges for misalignment and transverse deflections for bearings and gears are shown in Table 2. 
Table 2. Maximum Ranges for Slopes and Transverse Deflections (Shigley et al. 2003)

\begin{tabular}{ll}
\hline & Misalignment \\
\hline \hline Tapered Roller & $0.0005-0.0012 \mathrm{rad}$ \\
Cylinder Roller & $0.0008-0.0012 \mathrm{rad}$ \\
Deep-Groove Ball & $0.001-0.003 \mathrm{rad}$ \\
Spherical Ball & $0.026-0.052 \mathrm{rad}$ \\
Self-Aligned Ball & $0.026-0.052 \mathrm{rad}$ \\
Uncrowned Spur Gear & $<0.0005 \mathrm{rad}$ \\
\hline \hline
\end{tabular}

\subsubsection{Shaft Load Analysis}

\subsubsection{Three-Point Suspension Drivetrain}

Shaft geometry depends on the highest stresses experienced at stress concentration locations, typically at the main bearing locations. The first step in the shaft design is to determine the longitudinal direction given the transmitted torque and bending moments. Figure 2 shows the force diagram of a main shaft in a three-point suspension drivetrain. The following equations are derived based on the force and moment balance of the system.

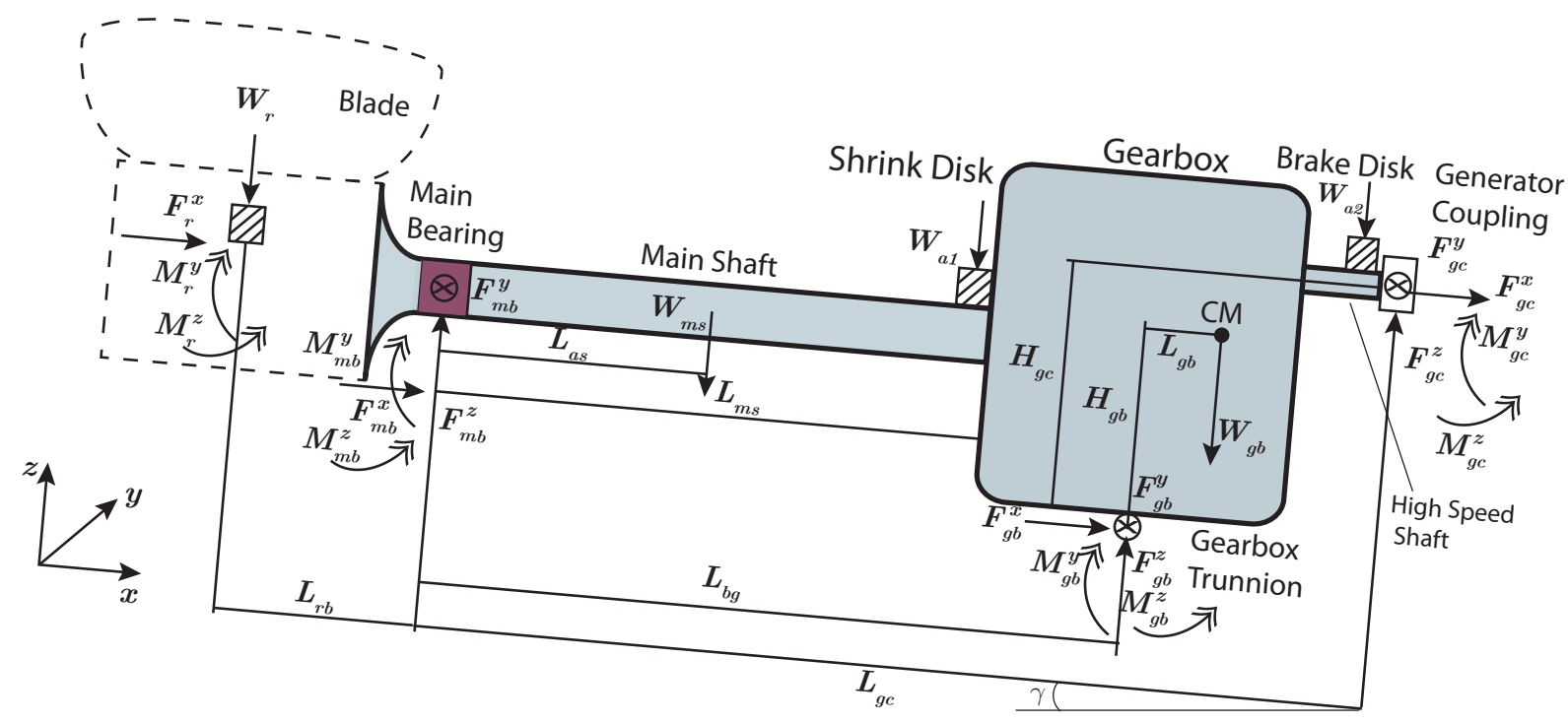

Figure 2. Force diagram of a main shaft in a three-point suspension drivetrain

The force balance along the $x$ axis $\sum F^{x}=0$ leads to:

$$
F_{r}^{x}+F_{m b}^{x}+F_{g b}^{x}+F_{g c}^{x}+\left(W_{r}+W_{m s}+W_{a 1}+W_{a 2}+W_{g b}\right) \sin (\gamma)=0
$$

The force balance along the $y$ axis $\sum F^{y}=0$ leads to:

$$
F_{r}^{y}+F_{m b}^{y}+F_{g b}^{y}+F_{g c}^{y}=0
$$

The force balance along the $z$ axis $\sum F^{z}=0$ leads to:

$$
F_{r}^{z}+F_{m b}^{z}+F_{g b}^{z}+F_{g c}^{z}-\left(W_{a 1}+W_{a 2}+W_{r}+W_{m s}+W_{g b}\right) \cos (\gamma)=0
$$


The balance of the pitching moments $\sum M^{y}=0$ around gearbox trunnions leads to:

$$
\begin{array}{r}
M_{r}^{y}+M_{m b}^{y}+F_{r}^{x} H_{g b}-W_{r} \cos (\gamma)\left(L_{r b}+L_{b g}\right)+F_{r}^{z} \cos (\gamma)\left(L_{r b}+L_{b g}\right) \\
+F_{m b}^{z} L_{b g}+F_{m b}^{x} H_{g b}-W_{m s}\left(L_{b g}-L_{a s}\right) \cos (\gamma)+W_{g b} \cos (\gamma) L_{g b}+M_{g b}^{y}+M_{g c}^{y} \\
-W_{a 1} \cos (\gamma)\left(L_{b g}-L_{m s}\right)+W_{a 2} \cos (\gamma)\left(L_{g c}-L_{b g}\right)+F_{g c}^{x} H_{g c}-F_{g c}^{z}\left(L_{g c}-L_{b g}\right)=0
\end{array}
$$

The balance of the yaw moments $\sum M^{z}=0$ around the gearbox trunnions leads to:

$$
M_{r}^{z}+M_{m b}^{z}-F_{r}^{y}\left(L_{b g}+L_{r b}\right)-F_{m b}^{y} L_{b g}+M_{g b}^{z}+M_{g c}^{z}+F_{g c}^{y}\left(L_{g c}-L_{b g}\right)=0
$$

Model assumptions are made based on system stiffness properties and common design criteria of individual components. For example, the radial stiffnesses of the main bearings are orders of magnitude higher than the tilting stiffnesses, resulting in higher radial loads than moments. Main bearings have large load capacity in the axial direction compared to gearbox bearings and support arms. They react to the rotor thrust and transfer it to the tower.

The main bearing does not carry moments, such that:

$$
M_{m b}^{y}=M_{m b}^{z}=0
$$

The generator coupling does not carry loads or moments; therefore:

$$
\begin{array}{r}
F_{g c}^{x}=F_{g c}^{y}=F_{g c}^{z}=0 \\
M_{g c}^{y}=M_{g c}^{z}=0
\end{array}
$$

Main shaft bending moments are carried by the main bearing and bearings of the gearbox low-speed stage, resulting in zero gearbox trunnion moments because of the trunnion's lower tilting stiffnesses:

$$
M_{g b}^{y}=M_{g b}^{z}=0
$$

The rotor axial loads are carried by the main bearing because the gearbox trunnion is compliant in the axial direction. The drivetrain axial loads caused by self-weight are carried by gearbox trunnions and generator coupling:

$$
F_{g c}^{x}+F_{g b}^{x}+\left(W_{m s}+W_{a 1}+W_{a 2}+W_{g b}\right) \sin (\gamma)=0
$$

By substituting Eqs. 2.9 and 2.12 into Eqs. 2.4 and Eq. 2.8, the solutions of Eqs. 2.4 and 2.8 can be derived. The loads at the main bearing and gearbox trunnions are:

$$
\left[\begin{array}{ll}
F_{m b}^{x} & =-F_{r}^{x}(t)-W_{r} \sin (\gamma) \\
F_{m b}^{y} & =\frac{M_{r}^{z}(t)}{L_{b g}}-\frac{F_{r}^{y}(t)\left(L_{b g}+L_{r b}\right)}{L_{b g}} \\
F_{m b}^{z} & =\frac{1}{L_{b g}}\left\{-M_{r}^{y}(t)+W_{r}\left[\cos (\gamma)\left(L_{r b}+L_{b g}\right)+\sin (\gamma) H_{g b}\right]-F_{r}^{z}(t) \cos (\gamma)\left(L_{r b}+L_{b g}\right)\right. \\
& \left.W_{m s}\left(L_{g b}-L_{a s}\right) \cos (\gamma)+W_{a 1} \cos (\gamma)\left(L_{b g}-L_{m s}\right)-W_{a 2} \cos (\gamma)\left(L_{g c}-L_{b g}\right)-W_{g b} \cos (\gamma) L_{g b}\right\} \\
F_{g b}^{x} & =-\left(W_{m s}+W_{a 1}+W_{a 2}+W_{g b}\right) \sin (\gamma) \\
F_{g b}^{y} & =-F_{m b}^{y}-F_{r}^{y}(t) \\
F_{g b}^{z} & =-F_{m b}^{z}+\left(W_{a 1}+W_{a 2}+W_{r}+W_{m s}+W_{g b}\right) \cos (\gamma)-F_{r}^{z}(t)
\end{array}\right.
$$

The bending moments along the main shaft in the pitching and yaw directions are:

$$
M_{y}(x)=\left[\begin{array}{l}
-F_{r}^{z}(t) x+W_{r} x-M_{r}^{y}(t)+\int_{0}^{x} \omega_{m s}(x) x \mathrm{~d} x, 0<x \leq L_{r b} \\
-F_{r}^{z}(t) x+W_{r} x-M_{r}^{y}(t)+\int_{0}^{x} \omega_{m s}(x) x \mathrm{~d} x-F_{m b}^{z}\left(x-L_{r b}\right), \quad L_{r b}<x \leq L_{r b}+L_{m s}
\end{array}\right.
$$

where $\omega_{m s}=\frac{W_{m s}}{L_{m s}}$.

$$
M_{z}(x)=\left[\begin{array}{l}
-M_{r}^{z}(t)-F_{r}^{y}(t) x, 0<x \leq L_{r b} \\
-M_{r}^{z}(t)-F_{r}^{y}(t) x-F_{m b}^{y}\left(x-L_{r b}\right), \quad L_{r b}<x \leq L_{r b}+L_{m s}
\end{array}\right.
$$




\subsubsection{Four-Point Suspension Drivetrain}

Figure 3 shows the force diagram of a main shaft in a four-point suspension drivetrain. The force and moment balance of the system in a four-point suspension is derived similarly to that of the three-point suspension system.

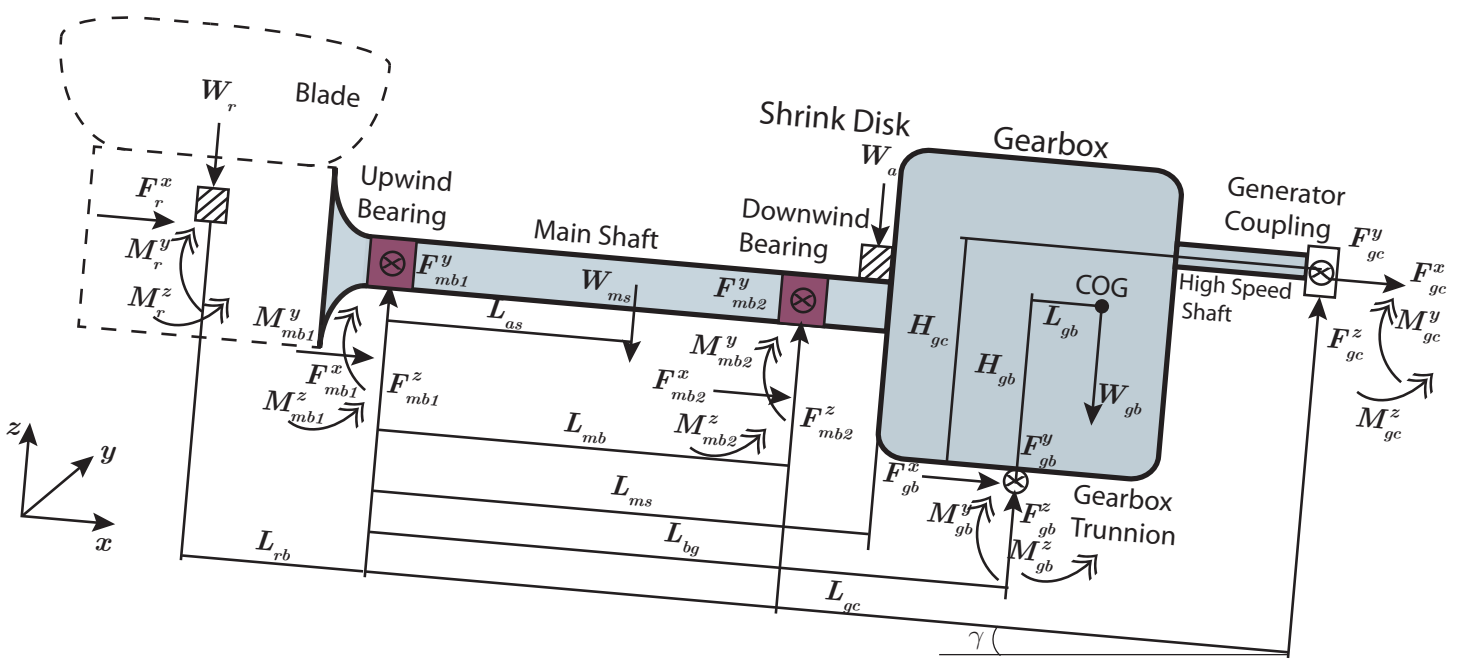

Figure 3. Force diagram of a main shaft in a four-point suspension drivetrain

Additional assumptions are made based on system stiffness properties in addition to those used for three-point drivetrains, as follows. The gearbox supports, flexible in tilting and axial directions, are designed to allow gearbox compliance in the associated directions.

1. Gearbox weight is carried by the trunnions:

$$
F_{g b}^{z}+W_{g b} \cos (\gamma)=0
$$

2. Gearbox trunnion force in the $y$ direction is negligible compared to the main bearing forces:

$$
\begin{aligned}
& F_{g b}^{z}+W_{g b} \cos (\gamma)=0 \\
& F_{g b}^{y}<<F_{m b 1}^{y}, F_{m b 2}^{y}
\end{aligned}
$$

The loads at the main bearings and gearbox trunnions are:

$$
\left[\begin{array}{ll}
F_{m b 2}^{x} & =-F_{r}^{x}(t)-W_{r} \sin (\gamma) \\
F_{m b 2}^{y} & =\frac{-M_{r}^{z}(t)+F_{r}^{y}(t) L_{r b}}{L_{m b}} \\
F_{m b 2}^{z} & =\frac{1}{L_{m b}}\left\{M_{r}^{y}(t)-W_{r} \cos (\gamma) L_{r b}-W_{m} s \cos (\gamma) L_{a s} W_{g b} \cos (\gamma) L_{g b}-W_{a 1} \cos (\gamma) L_{m s}-W_{a 2} \cos (\gamma) L_{g c}\right\} \\
F_{m b 1}^{x} & =0 \\
F_{m b 1}^{y} & =-F_{m b 2}^{y}-F_{r}^{y} \\
F_{m b 1}^{z} & =-F_{m b 2}^{z}+\left(W_{a 1}+W_{a 2}+W_{r}+W_{m s}\right) \cos (\gamma)-F_{r}^{z}(t)
\end{array}\right.
$$

The bending moments along the main shaft in the pitching and yaw directions are:

$$
\begin{gathered}
M_{y}(x)=\left[\begin{array}{l}
-F_{r}^{z}(t) x+W_{r} x-M_{r}^{y}(t)+\int_{0}^{x} \omega_{m s}(x) x \mathrm{~d} x, 0<x \leq L_{r b} \\
-F_{r}^{z}(t) x+W_{r} x-M_{r}^{y}(t)+\int_{0}^{x} \omega_{m s}(x) x \mathrm{~d} x-F_{m b 1}^{z}\left(x-L_{r b}\right), L_{r b}<x \leq L_{r b}+L_{m b} \\
-F_{r}^{z}(t) x+W_{r} x-M_{r}^{y}(t)+\int_{0}^{x} \omega_{m s}(x) x \mathrm{~d} x-F_{m b 1}^{z}\left(x-L_{r b}\right)-F_{m b 2}^{z}\left(x-L_{r b}-L_{m b}\right), L_{r b}+L_{m b}<x \leq L_{r b}+L_{m s}
\end{array}\right. \\
M_{z}(x)=\left[\begin{array}{l}
-M_{r}^{z}(t)-F_{r}^{y}(t) x, 0<x \leq L_{r b} \\
-M_{r}^{z}(t)-F_{r}^{y}(t) x-F_{m b 1}^{y}\left(x-L_{r b}\right), L_{r b}<x \leq L_{r b}+L_{m b} \\
-M_{r}^{z}(t)-F_{r}^{y}(t) x-F_{m b 1}^{y}\left(x-L_{r b}\right)-F_{m b 2}^{y}\left(x-L_{r b}-L_{m b}\right), L_{r b}+L_{m b}<x \leq L_{r b}+L_{m s}
\end{array}\right.
\end{gathered}
$$




\subsubsection{Shaft Dimension Determination}

The stresses at a point on the surface of a solid round shaft of diameter $d$ subject to bending, axial loading, and twisting are:

$$
\begin{array}{r}
\sigma_{x}=\frac{32\left(M_{y}^{2}+M_{z}^{2}\right) \frac{1}{2}}{\pi d^{3}}+\frac{4 F}{\pi d^{2}} \\
\tau_{x y}=\frac{16 T}{\pi d^{3}}
\end{array}
$$

By using a Mohr's circle, it can be shown that the two nonzero principal stresses are:

$$
\sigma_{a}, \sigma_{b}=\frac{\sigma_{x}}{2} \pm\left[\left(\frac{\sigma_{x}}{2}\right)^{2}+\tau_{x y}^{2}\right]^{\frac{1}{2}}
$$

These principal stresses can be combined to obtain the maximum shear stress, $\tau_{\max }$, and the von Mises stress, $\sigma^{\nu}$ :

$$
\begin{array}{ll}
\tau_{\max } & =\left[\left(\frac{\sigma_{x}}{2}\right)^{2}+\tau_{x y}{ }^{2}\right]^{\frac{1}{2}} \\
\sigma_{v} & =\left(\sigma_{x}{ }^{2}+3 \tau_{x y}^{2}\right)^{\frac{1}{2}}
\end{array}
$$

The allowable von Mises stress is:

$$
\sigma_{\text {all }}^{v}=\frac{S_{y}}{k}
$$

Under most conditions, the axial component, $F$, is either zero or so small that it can be neglected. With $F=0$ and Eq. 2.21 substituted into Eq. 2.23, Eq. 2.23 becomes:

$$
\begin{aligned}
& \tau_{\max }=\frac{16}{\pi d^{3}}\left(M_{y}^{2}+M_{z}^{2}+T^{2}\right)^{\frac{1}{2}} \\
& \sigma_{v}=\frac{16}{\pi d^{3}}\left(4 M_{y}^{2}+4 M_{z}^{2}+3 T^{2}\right)^{\frac{1}{2}}
\end{aligned}
$$

Using the distortion-energy theory of failure, the shaft diameter is solved as:

$$
d(x)=\frac{16 k}{\pi S_{y}}\left[4 M_{y}(x)^{2}+4 M_{z}(x)^{2}+3 T^{2}\right]^{\frac{1}{2}}
$$

where $M_{y}$ and $M_{z}$ are calculated from Eqs. 2.14 and 2.15 or Eqs. 2.19 and 2.20. The shaft inner diameter is assumed as $10 \%$ of the outer diameter. This value is selected based on the collected industry data.

Shaft deflection for the three-point suspension drivetrain is derived as:

$$
\left[\begin{array}{l}
M_{y}(x)=-F_{r}^{z}(t) x+W_{r} x-M_{r}^{y}(t)-F_{m b}^{z}\left(x-L_{r b}\right)+\int_{0}^{x} \omega_{m s}(x) x \mathrm{~d} x, L_{r b}<x \leq L_{r b}+L_{m s} \\
E I \frac{d v_{y}}{d x}=-\frac{F_{r}^{z}(t) x^{2}}{2}+W_{r} \frac{x^{2}}{2}-M_{r}^{y}(t) x-F_{m b}^{z} \frac{\left(x-L_{r b}\right)^{2}}{2}+\int_{y} \int_{x} \omega_{m s}(x) x \mathrm{~d} x \mathrm{~d} y+C_{1}, L_{r b}<x \leq L_{r b}+L_{m s} \\
E I v_{y}=-\frac{F_{r}^{z}(t) x^{3}}{6}+W_{r} \frac{x^{3}}{6}-M_{r}^{y}(t) \frac{x^{2}}{2}-F_{m b}^{z} \frac{\left(x-L_{r b}\right)^{3}}{6}+\int_{z} \int_{y} \int_{x} \omega_{m s}(x) x \mathrm{~d} x \mathrm{~d} y, \mathrm{~d} z+C_{1} x+C_{2}, L_{r b}<x \leq L_{r b}+L_{m s}
\end{array}\right.
$$

where the boundary conditions include:

$$
\begin{aligned}
& v_{y}=0, x=L_{r b} \\
& v_{y}=0, x+L_{r b}+L_{b g}
\end{aligned}
$$

Shaft deflection for the four-point suspension drivetrain is derived:

$$
\left[\begin{array}{ll}
M_{y}^{1}(x) & =-F_{r}^{z}(t) x+W_{r} x-M_{r}^{y}(t)+\int_{0}^{x} \omega_{m s}(x) x \mathrm{~d} x-F_{m b 1}^{z}\left(x-L_{r b}\right), L_{r b}<x \leq L_{r b}+L_{m b} \\
E I \frac{d v_{y}^{1}}{d x} & =-\frac{F_{r}^{z}(t) x^{2}}{2}+W_{r} \frac{x^{2}}{2}-M_{r}^{y}(t) x-F_{m b 1}^{z} \frac{\left(x-L_{r b}\right)^{2}}{2}+\int_{y} \int_{x} \omega_{m s}(x) x \mathrm{~d} x \mathrm{~d} y+D_{1} \\
E I v_{y}^{1} & =-\frac{F_{r}^{z}(t) x^{3}}{6}+W_{r} \frac{x^{3}}{6}-M_{r}^{y}(t) \frac{x^{2}}{2}-F_{m b 1}^{z} \frac{\left(x-L_{r b}\right)^{3}}{6}+\int_{z} \int_{y} \int_{x} \omega_{m s}(x) x \mathrm{~d} x \mathrm{~d} y, \mathrm{~d} z+D_{1} x+D_{2}
\end{array}\right.
$$

where the boundary conditions include:

$$
\begin{aligned}
& v_{y}^{1}=0, x=L_{r b} \\
& v_{y}^{1}=0, x+L_{r b}+L_{m b}
\end{aligned}
$$

The shaft length is optimized to be as long as possible until it cannot meet the deflection requirements at main bearings (refer to Table 2). With a relatively long shaft, the weights of the rear main bearing and gearbox structure can be reduced because of the decreased carried radial loads. The design process of the main shaft including main bearing(s) is illustrated in Figure 4. 


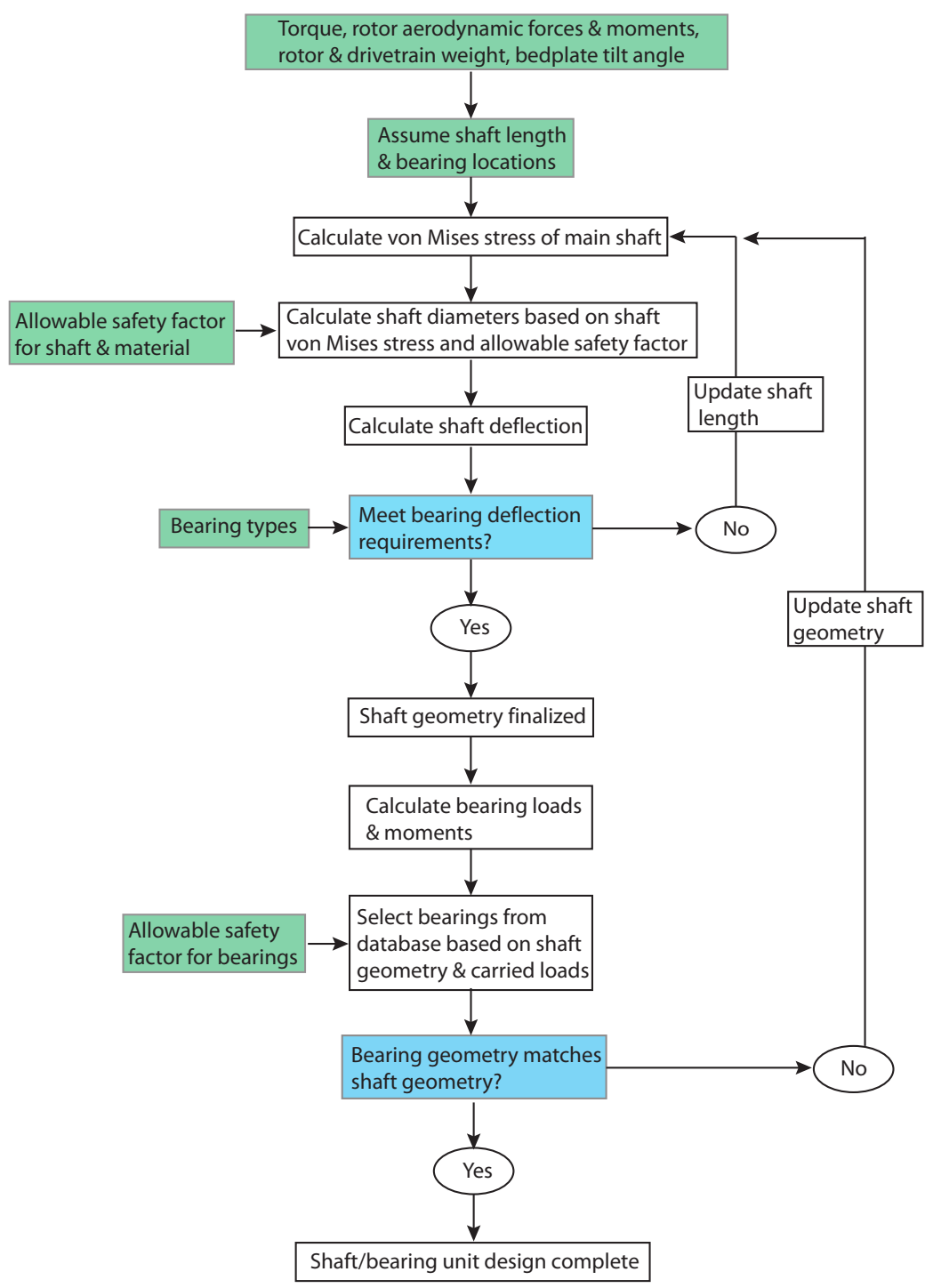

Figure 4. Flowchart of main shaft/bearing sizing model

\subsubsection{Mass Property Calculations}

The volume of the main shaft between the bearings is calculated using the following formula:

$$
\begin{aligned}
& \left.V_{m s}=\frac{\pi}{12}\left(d_{m b 1}{ }^{2}+d_{m b 2}{ }^{2}+d_{m b 2} d_{m b 1}\right) \quad L_{m b}-\frac{F W_{m b 1}+F W_{m b 2}}{2}\right] \quad \text { Volume of solid taper } \\
& +\frac{\pi}{4} d_{m b 1}{ }^{2} F W_{m b 1}+\frac{\pi}{4} d_{m b 1}{ }^{2} F W_{m b 1} \quad \text { Volume of shaft contained by main bearings } \\
& -\frac{\pi}{4} d_{m s, i}{ }^{2}\left(L_{m b}+\frac{F W_{m b 1}+F W_{m b 2}}{2}\right) \quad \text { Volume of hole }
\end{aligned}
$$

The mass of the main shaft is taken to be the density multiplied by the volume, where density of the shaft material is specified as $7,800 \mathrm{~kg} / \mathrm{m}^{3}$. The mass of the flange is then accounted for by multiplying this total mass by 1.33 (found from an average of solid model flange mass percentages). The total length of the main shaft takes into account the flange length, distance between main bearing centers, and facewidths in the following way: 


$$
L_{m s}=L_{m b}+\frac{F W_{m b 1}+F W_{m b 2}}{2}+L_{f}
$$

where $L_{f}$ is either specified by the user or, if unspecified, found from an approximate scaling argument with rotor diameter: $L_{f}=0.3{\frac{d_{r}}{100}}^{2}-0.1 \frac{d_{r}}{100}+0.4$.

The available solid models for both three-point and four-point main shafts all have a CM location that is very close to $65 \%$ of their length upwind from the gearbox connection. Using these data, the shaft CM is therefore modeled in the following way:

$$
\left[\begin{array}{ll}
C M_{m s}^{x} & =C M_{g b}^{x}-\frac{L_{g b}}{2}-0.65 L_{m s} \cos (\gamma) \\
C M_{m s}^{y} & =C M_{g b}^{y} \\
C M_{m s}^{z} & =C M_{g b}^{z}+0.65 L_{m s} \sin (\gamma)
\end{array}\right]
$$

\subsection{Main Bearings}

DriveSE lets users select from among six different bearing types: compact-aligning toroidal roller bearings (CARBs), spherical roller bearings (SRBs), single-row tapered roller bearings (TRBs1), double-row tapered roller bearings (TRBs2), cylindrical roller bearings (CRBs), and single-row deep-groove radial ball bearings (RBs). It is assumed that users are able to make their own judgments with respect to the bearing selection and configuration. After the final bearing has been selected, the model resizes the main shaft diameter(s) and bearing length(s) to fit the bore diameter and facewidths of the bearings. This completes the design of the shaft and bearing system.

Information on inner diameters, facewidth, load ratings, and masses are used to define the bearing selection in the model, which is collected from the SKF (2014) bearing database. With the shaft diameter and calculated dynamic load rating as inputs, the bearing sizing routine first determines if the load rating is higher than the load rating required to necessitate a larger, high-load bearing. If a high-load bearing is needed, the facewidth and mass of the bearing is determined from a curve fit of the data found in high- and low-load bins. An example of these interpolations for CARBs is shown in Figure 5. A chart of load rating versus bore diameter contains two distinct curves, one that can satisfy lower load requirements and one that has been shown to satisfy the requirements of bearings in higher load applications. A curve fit of the entire data set gives an averaged distribution, and divides the two curves into the low- and high-load bearings shown in Figure 6.

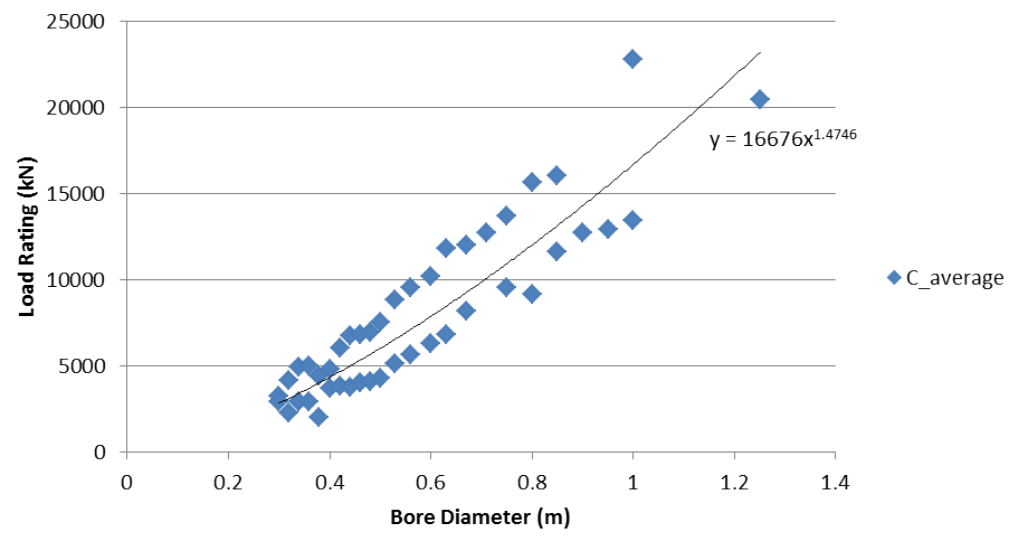

Figure 5. Dynamic load ratings for CARBs 


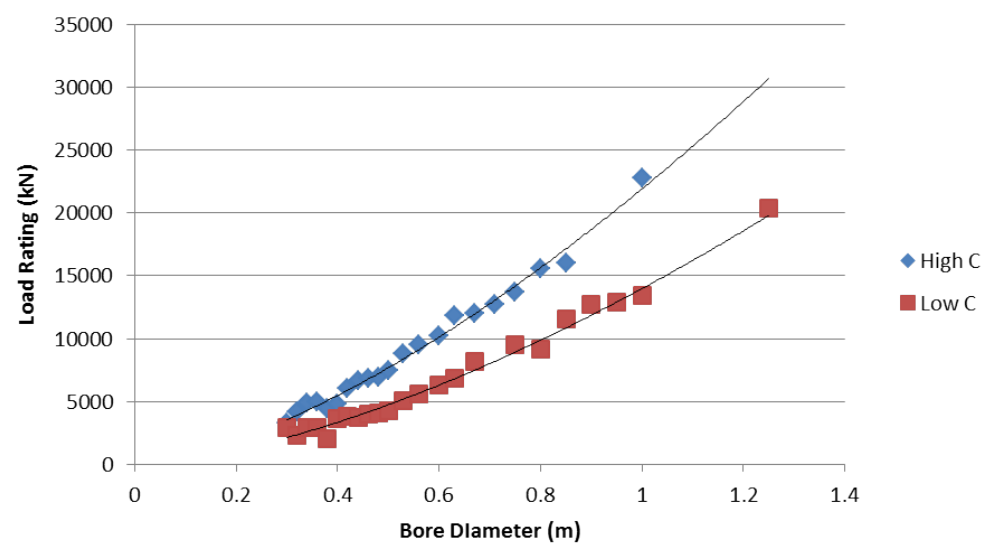

Figure 6. Dynamic load ratings for CARBs after load distinction

The two bearing types are then graphed against bore diameter in a similar way, showing facewidth and mass values with the new distinction between the selected high- and low-load bearings. The curves for CARBs are shown in Figure 7. From the diameter of the shaft at the bearing location, the predicted mass and facewidth of each bearing are selected using these two curves. The model typically selects bearings that follow the low-load curve.
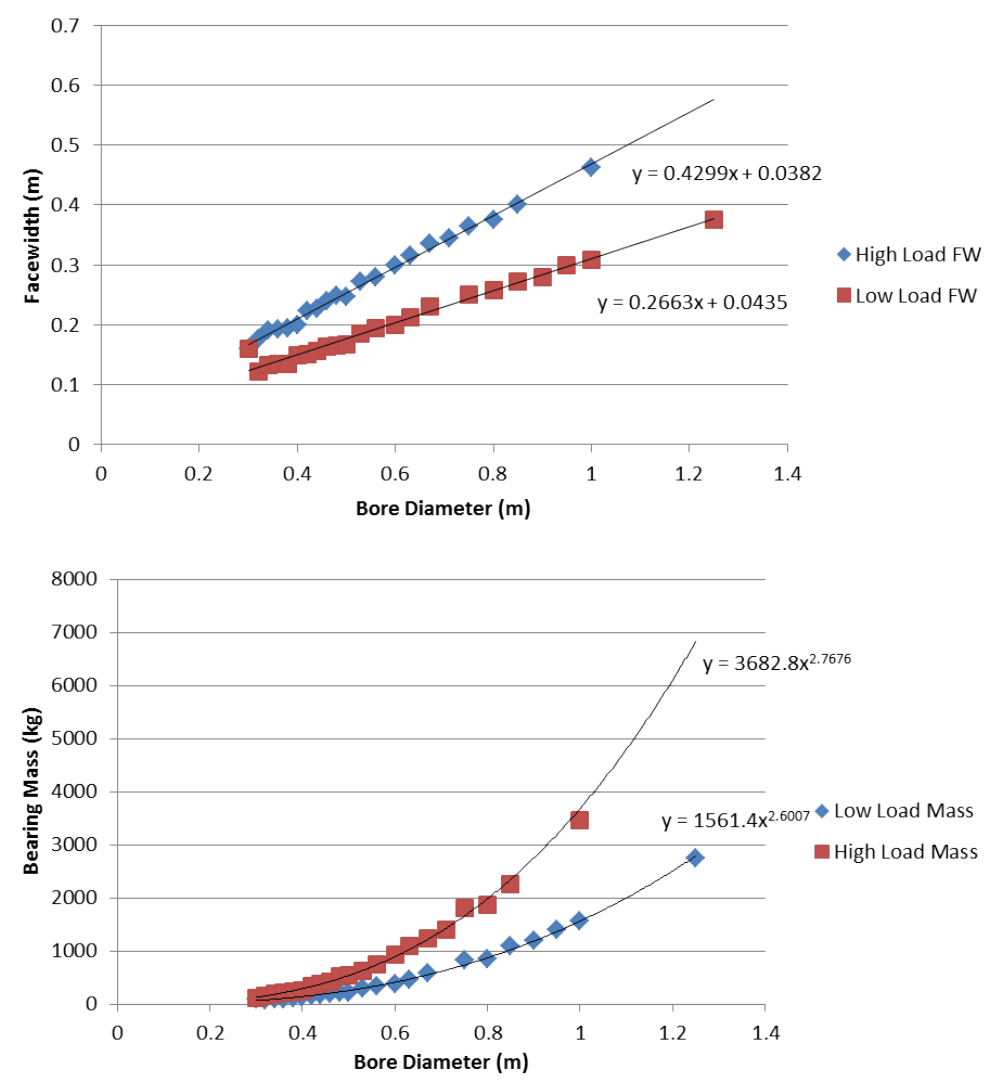

Figure 7. CARB mass and facewidth interpolation 
Not all bearing data from SKF follows a distribution of two load-based curves. In many cases, bearings of a similar diameter have four load ratings. To be consistent with all bearing types, the low- and high-load definitions are still used, and are taken to signify a middle ground between the low- and high-load distributions, detailed in Appendix E. In the future, a distinction might be made between curve fits of each load magnitude.

The bearing housing mass is calculated as $2.92 \times$ the bearing mass, then added to the bearings to obtain the entire bearing assembly mass. This is based on Sunderland model scaling relationships, but uses current industry data on contemporary bearing sizes.

Main bearing CM location(s) are defined by the distance between the main bearings and their relation to the gearbox location as follows:

$$
\left[\begin{array}{ll}
C M_{m b 1}^{x} & =C M_{g b x}^{x}-\frac{L_{g b x}}{2}-\left(L_{m b}+\frac{F W_{m b 2}}{2}\right) \cos (\gamma) \\
C M_{m b 1}^{y} & =C M_{g b x}^{y} \\
C M_{m b 1}^{z} & =C M_{g b x}^{z}+\left(L_{m b}+\frac{F W_{m b 2}}{2}\right) \sin (\gamma)
\end{array}\right]
$$

If a downwind main bearing exists, its $\mathrm{CM}$ is calculated in a similar way:

$$
\left[\begin{array}{ll}
C M_{m b 2}^{x} & =C M_{g b x}^{x}-\frac{L_{g b x}}{2}-\frac{F W_{m b 2}}{2} \cos (\gamma) \\
C M_{m b 2}^{y} & =C M_{g b x}^{y} \\
C M_{m b 2}^{z} & =C M_{g b x}^{z}+\frac{F W_{m b 2}}{2} \sin (\gamma)
\end{array}\right]
$$

\subsection{Gearbox}

A new design code is developed that uses turbine torque and overall speed ratio as input parameters. This model gives gear/bearing weight and housing weight and ratios per stage as well as overall gearbox weight. The size of the gearbox is determined for different gearbox configurations for minimizing the gearbox weight. Figure 8 shows major steps for the developed sizing model.

The gearbox model includes an internal design optimizer to reduce its weight. It selects the best combination of speed ratios for each gear stage to achieve the lowest weight, given the user-specified overall speed ratio of the entire gearbox. This sizing model is also suitable for a sensitivity study of gearbox weight compared to various design parameters. The parameters of interest are the number of stages, number of planets in planetary gears, gearbox configuration, and overall speed ratio. Gearbox design constraints such as the maximum number of pinion teeth and maximum facewidth/gear pitch diameter ratios discussed in Schultz (2009) are not considered in DriveSE currently.

Input torque drives wind turbine gearbox design. Influences of nontorque loads caused by rotor overhang weight and aerodynamic forces on gearbox weight are considered in this work. For three-point suspension gearboxes, a factor of 1.25 is multiplied to the planetary gear weight to take into account the nontorque loads applied on the gearbox. This factor is selected based on Gearbox Reliability Collaborative (GRC) measured load sharing factor in the upwind planetary gear section. For other drivetrain configurations, nontorque loads are small and therefore not considered in the model. The gearbox rating (bending and pitting resistance) analysis is not the focus of this approach, so the resulting changes to the gearbox that would stem from these design drivers are not included.

This model focuses on the design of three-stage gearboxes with common configurations: one planetary gear and two parallel stages, and two planetary gears and one parallel stage.

\subsubsection{Sizing of a Single External Gear}

The relationship between the overall gear dimensions; the speed ratio, $U_{s}$; and power, $P$, for external gears is discussed in (Dudley 1984):

$$
C^{2} F=\frac{31,500 P\left(U_{s}+1\right)^{3}}{K n_{p} U_{s}}
$$

where $C=0.5 d_{p}\left(U_{s}+1\right)$ is the center distance and $d_{p}$ and $F$ are the gear diameter and facewidth. The transmitted power $P=$ $\frac{T n_{p}}{63,000}$ is linearly correlated to the gearbox torque, $T$, and speed, $n_{p} . K$ factor is an index for measuring the intensity of tooth loads (Dudley 1984). There are different ways to calculate the $K$ factor: (1) it can be estimated from the empirical table (2.45) in (Dudley 1984); or (2) it can be calculated by Eq. 2.37 when the gearbox component dimensions are designed: 


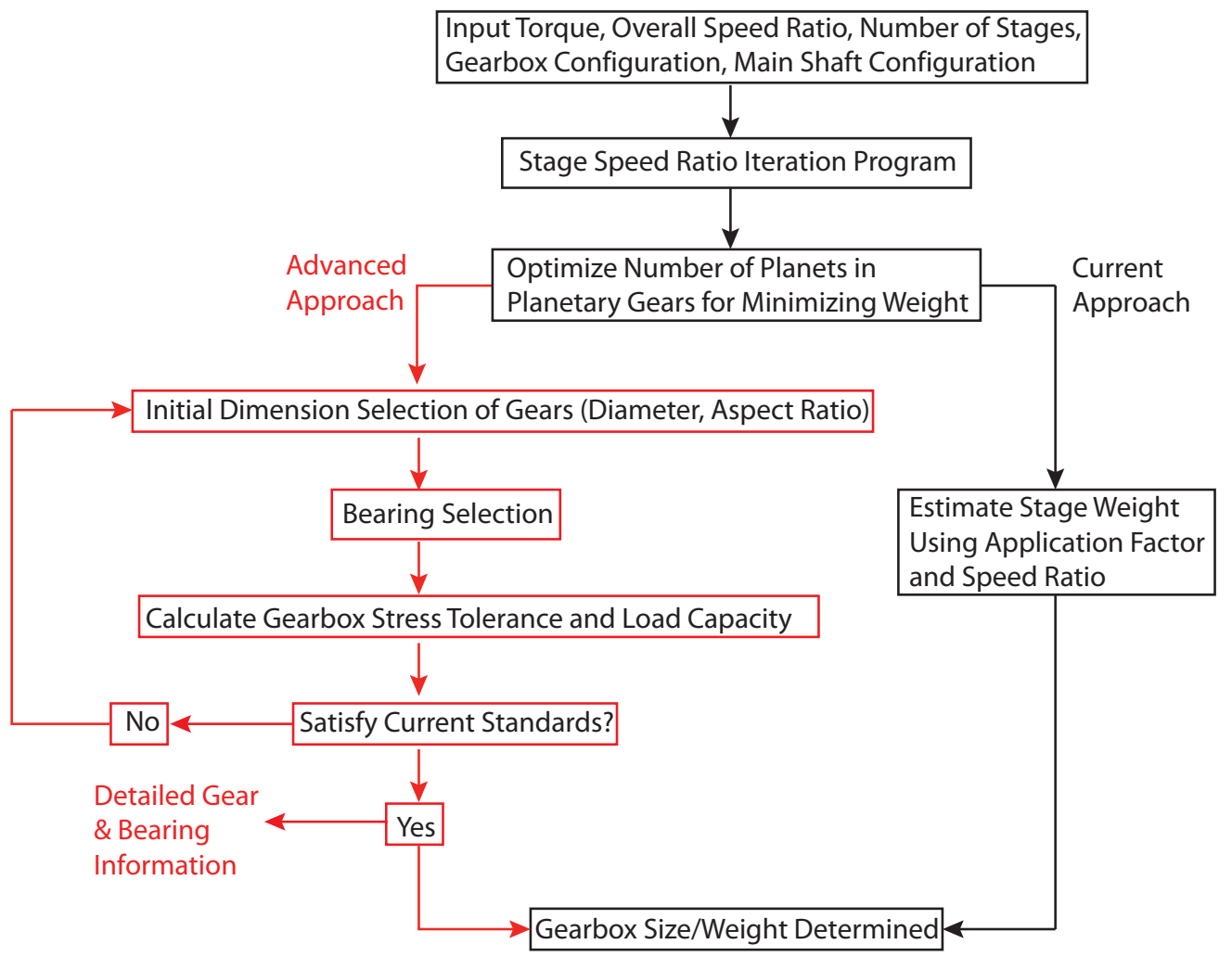

Figure 8. Flowchart of the gearbox sizing tool

$$
K=\frac{W_{t}}{F W d} \frac{U_{s}+1}{U_{s}}
$$

where $W_{t}=\frac{2 Q_{p}}{d_{p}}$ is the tangential driving force, and $Q_{p}$ is the inft torque to the pinion.

In this study, the first approach is used.

Rewriting Eq. 2.36 results in:

$$
F d_{p}^{2}=\frac{2 Q_{p}}{K} \frac{U_{s}+1}{U_{s}}
$$

The gearbox stage weight is estimated by $W_{G B}=K_{A G} F d_{p}^{2}$, where $K_{A G}$ is the application factors for weight estimations (Willis 1963). The final form is:

$$
W_{G B}=K_{A G} \frac{2 Q_{p}}{K} \frac{U_{s}+1}{U_{s}}
$$

\subsubsection{Sizing of an External Gear Pair}

The driving pinion volume equals $F W d_{p}^{2}$. The driven gear volume is $F W d_{p}^{2} U_{s}^{2}$. Therefore, the total size of the gear pair equals:

$$
\sum F W d^{2}=F W d_{p}^{2}+F W d_{p}^{2} U_{s}^{2}
$$

The total weight of the gear pair equals:

$$
\begin{aligned}
W_{G B P N} & =K_{A G} \frac{2 Q_{p}}{K}\left(\frac{U_{s}+1}{U_{s}}\right)+K_{A G} \frac{2 Q_{p}}{K}\left(\frac{U_{s}+1}{U_{s}}\right) U_{s}^{2} \\
& =K_{A G} \frac{2 Q_{p}}{K}\left(1+\frac{1}{U_{s}}+U_{s}+U_{s}^{2}\right)
\end{aligned}
$$




\subsubsection{Sizing of a Planetary Gear Stage}

The volume of a planetary gear consists of the sun, ring, and $B$ planet gears. The sun gear volume is:

$$
F W d_{s}^{2}=\frac{2 Q_{s}}{B K}\left(\frac{U_{S N}+1}{U_{S N}}\right)
$$

where $U_{S N}=0.5 U_{s}-1$ is the speed ratio between the sun and planet and $Q_{s}$ is the input torque to the sun gear.

The volume of a planet is:

$$
F W d_{p}^{2}=F W d_{s}^{2} U_{S N}^{2}=\frac{2 Q_{s}}{B_{p} K}\left(\frac{U_{S N}+1}{U_{S N}}\right) U_{S N}^{2}
$$

The volume of the ring gear depends on both its diameter and thickness. AGMA 6123 (AGMA 2006) defines the ring thickness as no less than three times the module thickness. The ring gear volume is approximated empirically without designing individual gear dimensions. The ring volume considers the weight of the housing and carrier:

$$
V_{r g}=K_{r} F W d_{s}^{2}\left(\frac{d_{r} g}{d_{s}}\right)^{2}=K_{r} \frac{2 Q_{s}}{B_{p} K}\left(\frac{U_{S N}+1}{U_{S N}}\right)\left(\frac{d_{r} g}{d_{s}}\right)^{2}
$$

where $K_{r}=0.4$ is the scaling factor, selected from (Willis 1963).

Therefore, the overall planetary gear volume is:

$$
\begin{aligned}
F W d_{s}^{2}+B_{p} F W d_{p}^{2}+V_{r g} & =\frac{2 Q_{s}}{B_{p} K}\left(\frac{U_{S N}+1}{U_{S N}}\right)+B_{p} \frac{2 Q_{s}}{B_{p} K}\left(\frac{U_{S N}+1}{U_{S N}}\right) U_{S N}^{2}+K_{r} \frac{2 Q_{s}}{B_{p} K}\left(\frac{U_{S N}+1}{U_{S N}}\right)\left(\frac{d_{r} g}{d_{s}}\right)^{2} \\
& =\frac{2 Q_{s}}{B_{p} K}\left[\frac{1}{B_{p}}+\frac{1}{B_{p} U_{S N}}+U_{S N}+U_{S N}^{2}+K_{r} \frac{\left(U_{s}-1\right)^{2}}{B_{p}}+K_{r} \frac{\left(U_{s}-1\right)^{2}}{B_{p} U_{s}}\right]
\end{aligned}
$$

The planetary gear weight equals:

$$
W_{G S E N}=K_{A G} \frac{2 Q_{s}}{K}\left[\frac{1}{B_{p}}+\frac{1}{B_{p} U_{S N}}+U_{S N}+U_{S N}^{2}+K_{r} \frac{\left(U_{s}-1\right)^{2}}{B_{p}}+K_{r} \frac{\left(U_{s}-1\right)^{2}}{B_{p} U_{S N}}\right]
$$

\subsubsection{Gearbox Total Weight}

The gearbox weight is the summation of individual stage weight, which depends on the input torque, $Q_{1}, Q_{2}, Q_{3}$, and speed ratio, $U_{1}, U_{2}, U_{3} \cdot Q_{0}=Q_{1} U_{1} \cdot Q_{1}=Q_{2} U_{2} \cdot Q_{2}=Q_{3} U_{3}$, where $Q_{0}$ is the main shaft input torque. For example, the $750-\mathrm{kW}$ gearbox (Link et al. 2011) utilizes a planetary-parallel-parallel (epp) configuration. The total weight of this gearbox is:

$$
\begin{aligned}
W_{G B} & =W_{G S E N}^{1}+W_{G B P N}^{2}+W_{G B P N}^{3} \\
& =K_{A G} \frac{2 Q_{1}}{K}\left[\frac{1}{B_{p}}+\frac{1}{B_{p} U_{S N}}+U_{S N}+U_{S N}^{2}+K_{r} \frac{\left(U_{1}-1\right)^{2}}{B_{p}}+K_{r} \frac{\left(U_{1}-1\right)^{2}}{B_{p} U_{1}}\right] \\
& +K_{A G} \frac{2 Q_{2}}{K}\left(1+\frac{1}{U_{2}}+U_{2}+U_{2}^{2}\right)+K_{A G} \frac{2 Q_{3}}{K}\left(1+\frac{1}{U_{3}}+U_{3}+U_{3}^{2}\right)
\end{aligned}
$$

The final gearbox design takes into account the gear dynamic effects on loads, overload, unequal load sharing for planetary gears, and the main shaft configuration; therefore, the gearbox weight considers the overload factor, $K_{0}$ (Avallone et al. 2006); dynamic factor, $K_{v}$ (AGMA 2010); load sharing factor among planets, $K_{\gamma p}$ (ISO 2012); load-sharing factors between bearing rows, $K_{\gamma b}$; and a new factor that captures the effects of the main shaft configurations on gearbox loads, $K_{S H}$. In the model, $K_{S H}=1$ is used as the default.

$$
W_{G B}^{0}=K_{0} K_{v} K_{\gamma p} K_{\gamma b} K_{S H} W_{G B}
$$

\subsubsection{Gearbox Speed Ratio Optimization}

This method selects the optimal speed ratios of individual gear stages for minimizing gearbox weight.

\section{Gearboxes With Two Parallel Stages}


The total volume of the gearbox is proportional to:

$$
\begin{aligned}
\Sigma F d^{2} & =F W_{1} d_{p 1}^{2}+F W_{1} d_{g 1}^{2}+F W_{2} d_{p 2}^{2}+F W_{2} d_{g 2}^{2} \\
& =K_{A G} \frac{2 Q_{1}}{K}\left(1+\frac{1}{U_{1}}+U_{1}+U_{1}^{2}\right)+K_{A G} \frac{2 Q_{2}}{K}\left(1+\frac{1}{U_{2}}+U_{2}+U_{2}^{2}\right) \\
& =K_{A G} \frac{2 Q_{1}}{K}\left(1+\frac{1}{U_{1}}+U_{1}+U_{1}^{2}\right)+K_{A G} \frac{2 Q_{1} U_{1}}{K}\left(1+\frac{U_{1}}{M_{0}}+\frac{M_{0}}{U_{1}}+{\frac{M_{0}}{U_{1}}}^{2}\right)
\end{aligned}
$$

For minimum volume, set the derivative to zero:

$$
\frac{d\left(\sum F W d^{2}\right)}{d U_{1}}=-\frac{1}{U_{1}^{2}}+2+2 U_{1}+2 \frac{U_{1}}{M_{0}}-\frac{M_{0}^{2}}{U_{1}^{2}}=0
$$

An iteration program is needed to find the roots of Eq. 2.50.

\section{Gearboxes With Three Stages}

Two primary configurations for three-stage gearboxes are included in the current version of the model: planetary-parallel-parallel (epp) and planetary-planetary-parallel (eep).

\section{Planetary-Parallel-Parallel Configuration}

The total volume of the gearbox equals:

$$
\begin{aligned}
V= & \left.\frac{2 Q_{0}}{K} \frac{1}{U_{1}} \frac{1}{B_{1}}+\frac{1}{B_{1}\left(\frac{U_{1}}{2}-1\right)}+\left(\frac{U_{1}}{2}-1\right)+\left(\frac{U_{1}}{2}-1\right)^{2}+K_{r} \frac{\left(U_{1}-1\right)^{2}}{B_{1}}+K_{r} \frac{\left(U_{1}-1\right)^{2}}{B_{1}\left(\frac{U}{2}-1\right)}\right] \\
& +\frac{2 Q_{0}}{K} \frac{1}{U_{1} U_{2}} 1+\frac{1}{U_{2}}+U_{2}+U_{2}^{2}+\frac{2 Q_{0}}{K} \frac{1}{U_{1} U_{2} U_{3}} 1+\frac{1}{U_{3}}+U_{3}+U_{3}^{2}
\end{aligned}
$$

Let $M_{1}=U_{1} U_{2}, M_{2}=U_{2} U_{3}$, and $M_{0}=U_{1} U_{2} U_{3}$, and rewrite Eq. 2.51 as:

$$
\begin{aligned}
V\left(M_{1}, U_{1}\right)= & \left.\frac{2 Q_{0}}{K} \frac{1}{U_{1}} \frac{1}{B_{1}}+\frac{1}{B_{1}\left(\frac{U_{1}}{2}-1\right)}+\left(\frac{U_{1}}{2}-1\right)+\left(\frac{U_{1}}{2}-1\right)^{2}+K_{r} \frac{\left(U_{1}-1\right)^{2}}{B_{1}}+K_{r} \frac{\left(U_{1}-1\right)^{2}}{B_{1}\left(\frac{U}{2}-1\right)}\right] \\
& +\frac{2 Q_{0}}{K} \frac{1}{M_{1}} 1+\frac{U_{1}}{M_{1}}+\frac{M_{1}}{U_{1}}+\left(\frac{M_{1}}{U_{1}}\right)^{2}+\frac{2 Q_{0}}{K} \frac{1}{M_{0}} 1+\frac{M_{1}}{M_{0}}+\frac{M_{0}}{M_{1}}+\left(\frac{M_{0}}{M_{1}}\right)^{2} \\
V\left(M_{2}, U_{2}\right)= & \left.\frac{2 Q_{0}}{K} \frac{M_{2}}{M_{0}} \frac{1}{B_{1}}+\frac{1}{B_{1}\left(\frac{M_{0}}{2 M_{2}}-1\right)}+\left(\frac{M_{0}}{2 M_{2}}-1\right)+\left(\frac{M_{0}}{2 M_{2}}-1\right)^{2}+K_{r} \frac{\left(\frac{M_{0}}{M_{2}}-1\right)^{2}}{B_{1}}+K_{r} \frac{\left(\frac{M_{0}}{M_{2}}-1\right)^{2}}{B_{1}\left(\frac{M_{0}}{2 M_{2}}-1\right)}\right] \\
& +\frac{2 Q_{0}}{K} \frac{M_{2}}{M_{0} U_{2}} 1+\frac{1}{U_{2}}+U_{2}+U_{2}^{2}+\frac{2 Q_{0}}{K} \frac{1}{M_{0}} 1+\frac{U_{2}}{M_{2}}+\frac{M_{2}}{U_{2}}+\left(\frac{M_{2}}{U_{2}}\right)^{2}
\end{aligned}
$$

The gearbox volume reaches the minimum when $\frac{d V\left(M_{1}, U_{1}\right)}{d U_{1}}=0$ and $\frac{d V\left(M_{2}, U_{2}\right)}{d U_{2}}=0$ :

$$
\begin{gathered}
\frac{d V\left(M_{1}, U_{1}\right)}{d U_{1}}=\quad \frac{1}{M_{1}^{2}}+\frac{(N p+4 K r)}{4 B_{p}} U_{1}^{3}\left(\frac{U}{2}-1\right)^{2}-\frac{\left(K r+B_{p}+1\right)}{B_{p}} U_{1}\left(\frac{U_{1}}{2}-1\right)^{2} \\
-2 M_{1}\left(\frac{U_{1}}{2}-1\right)^{2}+\frac{2 K_{r}\left(U_{1}-1\right) U_{1}^{2}\left(\frac{U}{2}-1\right)}{B_{p}}-\frac{\left(K_{r}\left(U_{1}-1\right)^{2}+1\right) U_{1}\left(U_{1}-1\right)}{B_{p}} \\
\frac{d V\left(M_{2}, U_{2}\right)}{d U_{2}}=\frac{\left(M_{2}^{2}+1\right)}{M_{0} M_{2} U_{2}^{3}}-\frac{2 M_{2}}{M_{0} U_{2}}-\frac{2 M_{2}}{M_{0}\left(M_{2}+1\right)}
\end{gathered}
$$

\section{Planetary-Planetary-Parallel Configuration}

The total volume of the gearbox equals:

$$
\begin{aligned}
V= & \left.\frac{2 Q_{0}}{K} \frac{1}{U_{1}} \frac{1}{B_{1}}+\frac{1}{B_{1}\left(\frac{U_{1}}{2}-1\right)}+\left(\frac{U_{1}}{2}-1\right)+\left(\frac{U_{1}}{2}-1\right)^{2}+K_{r 1} \frac{\left(U_{1}-1\right)^{2}}{B_{1}}+K_{r 1} \frac{\left(U_{1}-1\right)^{2}}{B_{1}\left(\frac{U_{1}}{2}-1\right)}\right] \\
& \left.+\frac{2 Q_{0}}{K} \frac{1}{U_{1} U_{2}} \frac{1}{B_{2}}+\frac{1}{B_{2}\left(\frac{U_{2}}{2}-1\right)}+\left(\frac{U_{2}}{2}-1\right)+\left(\frac{U_{2}}{2}-1\right)^{2}+K_{r 2} \frac{\left(U_{2}-1\right)^{2}}{B_{2}}+K_{r 2} \frac{\left(U_{2}-1\right)^{2}}{B_{2}\left(\frac{U_{2}}{2}-1\right)}\right] \\
& +\frac{2 Q_{0}}{K} \frac{1}{U_{1} U_{2} U_{3}} 1+\frac{1}{U_{3}}+U_{3}+U_{3}^{2}
\end{aligned}
$$


Let $M_{1}=U_{1} U_{2}, M_{2}=U_{2} U_{3}$, and $M_{0}=U_{1} U_{2} U_{3}$, and rewrite Eq. 2.56 as:

$$
\begin{aligned}
V\left(M_{1}, U_{1}\right)= & \frac{2 Q_{0}}{K} \frac{1}{U_{1}} \frac{1}{B_{1}}+\frac{1}{B_{1}\left(\frac{U_{1}}{2}-1\right)}+\left(\frac{U_{1}}{2}-1\right)+\left(\frac{U_{1}}{2}-1\right)^{2}+K_{r 1} \frac{\left(U_{1}-1\right)^{2}}{B_{1}}+K_{r 1} \frac{\left(U_{1}-1\right)^{2}}{B_{1}\left(\frac{U_{1}}{2}-1\right)} \\
& +\frac{2 Q_{0}}{K} \frac{1}{M_{1}} \frac{1}{B_{2}}+\frac{1}{B_{2}\left(\frac{M_{1}}{2 U_{1}}-1\right)}+\left(\frac{M_{1}}{2 U_{1}}-1\right)+\left(\frac{M_{1}}{2 U_{1}}-1\right)^{2}+K_{r 2} \frac{\left(\frac{M_{1}}{U_{1}}-1\right)^{2}}{B_{2}}+K_{r 2} \frac{\left(\frac{M_{1}}{U_{1}}-1\right)^{2}}{B_{2}\left(\frac{M_{1}}{2 U_{1}}-1\right)} \\
& +\frac{2 Q_{0}}{K} \frac{1}{M_{0}} 1+\frac{M_{1}}{M_{0}}+\frac{M_{0}}{M_{1}}+\left(\frac{M_{0}}{M_{1}}\right)^{2} \\
V\left(M_{2}, U_{2}\right)= & \frac{2 Q_{0}}{K} \frac{M_{2}}{M_{0}} \frac{1}{B_{1}}+\frac{1}{B_{1}\left(\frac{M_{0}}{2 M_{2}}-1\right)}+\left(\frac{M_{0}}{2 M_{2}}-1\right)+\left(\frac{M_{0}}{2 M_{2}}-1\right)^{2}+K_{r 1} \frac{\left(\frac{M_{0}}{M_{2}}-1\right)^{2}}{B_{1}}+K_{r 1} \frac{\left(\frac{M_{0}}{M_{2}}-1\right)^{2}}{B_{1}\left(\frac{M_{0}}{2 M_{2}}-1\right)} \\
& +\frac{2 Q_{0}}{K} \frac{M_{2}}{M_{0} U_{2}} \frac{1}{B_{2}}+\frac{1}{B_{2}\left(\frac{U_{2}}{2}-1\right)}+\left(\frac{U_{2}}{2}-1\right)+\left(\frac{U_{2}}{2}-1\right)^{2}+K_{r 2} \frac{\left(U_{2}-1\right)^{2}}{B_{2}}+K_{r 2} \frac{\left(U_{2}-1\right)^{2}}{B_{2}\left(\frac{U_{2}}{2}-1\right)} \\
& +\frac{2 Q_{0}}{K} \frac{1}{M_{0}} 1+\frac{U_{2}}{M_{2}}+\frac{M_{2}}{U_{2}}+\left(\frac{M_{2}}{U_{2}}\right)^{2}
\end{aligned}
$$

The derivatives are then:

$$
\begin{gathered}
\frac{d V\left(M_{1}, U_{1}\right)}{d U_{1}}=-\frac{\left(U_{1}-1\right)\left(1+K_{r}\left(U_{1}-1\right)^{2}\right)}{B_{1} U_{1}^{2}\left(\frac{U_{1}}{2}-1\right)^{2}}+\frac{2 K_{r}\left(U_{1}-1\right)}{B_{1} U_{1}\left(\frac{U_{1}}{2}-1\right)}-\frac{\left(1+K_{r}\right)}{B_{1} U_{1}^{2}}+\frac{1}{4}+\frac{K_{r}}{B_{1}}+\frac{1}{2 B_{2}\left(\frac{M_{1}}{2}-U_{1}\right)^{2}} \\
-\frac{2 K_{r}\left(M_{1}-U_{1}\right)}{B_{2} U_{1}^{2}\left(\frac{M_{1}}{2}-U_{1}\right)}+\frac{K_{r}\left(M_{1}-U_{1}\right)^{2}}{2 B_{2} U_{1}^{2}\left(\frac{M_{1}}{2}-U_{1}\right)^{2}}+\left(\frac{1}{2}+\frac{2 K_{r}}{B_{2}}\right)\left(\frac{1}{U_{1}^{2}}-\frac{M_{1}}{U_{1}^{3}}\right) \\
\frac{d V\left(M_{2}, U_{2}\right)}{d U_{2}}=-\frac{M_{2}\left(U_{2}-1\right)\left(1+K_{r} M_{2}\left(U_{2}-1\right)^{2}\right)}{M_{0} B_{2} U_{2}^{2}\left(\frac{U_{2}}{2}-1\right)^{2}}+\frac{2 K_{r} M_{2}\left(U_{2}-1\right)}{M 0 B_{2} U_{2}\left(\frac{U_{2}}{2}-1\right)} \\
+\left(\frac{M_{2}}{4 M_{0}}+\frac{K_{r} M}{M_{0} B_{2}}+\frac{1}{M_{2} M_{0}}\right)+\frac{\left(-\frac{M_{2}}{M_{0} B_{2}}-\frac{K_{r} M}{M_{0} B_{2}}-\frac{M_{2}}{M_{0}}\right)}{U_{2}^{2}}-\frac{2 M^{2}}{M_{0} U_{2}^{3}}
\end{gathered}
$$

$\frac{d V\left(M_{1}, U_{1}\right)}{d U_{1}}, \frac{d V\left(M_{1}, U_{1}\right)}{d U_{1}}$ can also be calculated numerically by using finite differencing. Central difference formulation $\frac{d V(U+d U)-d V(U-d U)}{2 d U}$ (second-order accuracy) is used here to check the accuracy of the analytical formulations in Eqs. 2.54, 2.55, 2.59, and 2.60. $d U=2 \times 10^{-5}$ is selected through a sensitivity test to find the optimal step size to avoid machine round-off.

\section{Optimizer Solving Approach}

An iterative process is used to solve gearbox stage ratios (the roots of Eqs. 2.59 and 2.60: $U_{1}$ and $U_{2}$ for a given $M_{1}=U_{1} U_{2}$ ) that can result in the minimum weight. This process searches stage ratios with which the first derivatives of the gearbox volume equal zero:

1. Select an initial value for $M_{1}=U_{1} U_{2}$, by default is selected as $M_{0} / 2.5$, where $M_{0}$ is total gear ratio specified by users.

2. Set the derivative of the gearbox volume to zero: $\frac{\partial V_{G B}}{\partial U_{1}}=0$.

3. Solve for the root $U_{1}$.

4. Calculate $U_{2}=M_{1} / U_{1}$.

5. Calculate $U_{3}=M_{0} / M_{1}$.

6. Recalculate $M_{2}=U_{2} U_{3}$.

7. Solve for $U_{2}$ from $\frac{\partial V_{G B}}{\partial U_{2}}=0$.

8. Update $M_{1}=U_{1} U_{2}$.

9. Iterate until $U_{2}$ from the last step equals $U_{2}$ from step 4 .

The convergence tolerance used in the iteration is $5 \times 10^{-3}$. This iterative process is also illustrated in Figure 9 .

\subsubsection{Mass Property Calculations}

After finding the mass via the approach corresponding to the selected gearbox configuration as described previously, the next step is to calculate the mass properties for the gearbox. The gearbox CM is found using the user-input x-location (defaulted above tower top center), and the height of the gearbox, as shown in Eq. 2.61: 


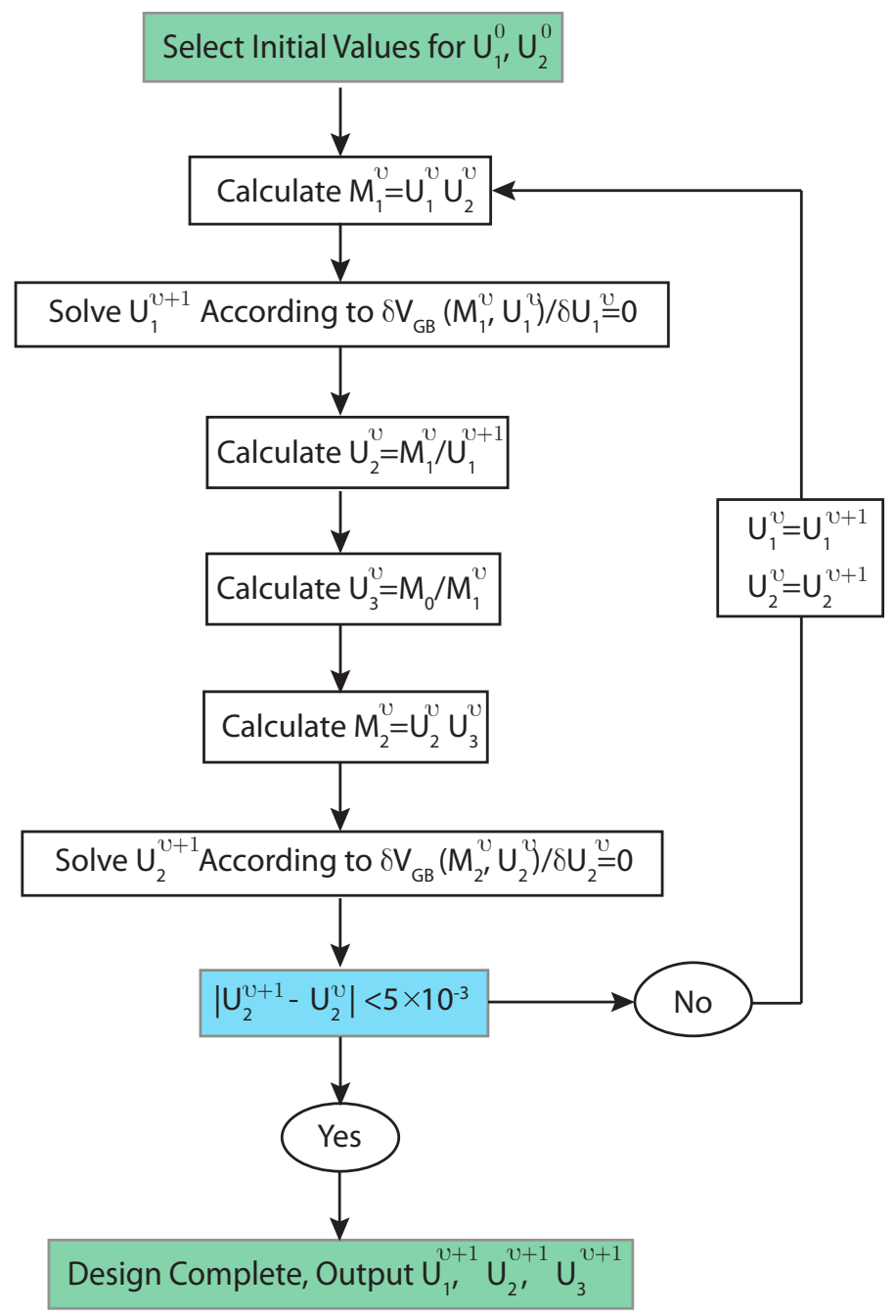

Figure 9. Flowchart of the gearbox iteration process for determining the stage ratios

$$
\left[\begin{array}{ll}
C M_{g b x}^{x} & =0 \text { or user input } \\
C M_{g b x}^{y} & =0 \\
C M_{g b x}^{z} & =\frac{H_{g b x}}{2}
\end{array}\right]
$$

where the gearbox dimensions still rely on scaling arguments based on NacelleSE, as follows:

$$
\begin{aligned}
& L_{g b x}=0.012 \times D_{r} \\
& H_{g b x}=0.015 \times D_{r}
\end{aligned}
$$

\subsection{Yaw System}

DriveSE assumes that a friction plate yaw bearing at the nacelle tower interface and several yaw motors comprise the yaw system. The friction plate bearing is treated as a steel annulus and is sized according to the tower top diameter and rotor diameter. The motors are assumed to be a common Bonfiglioli design from the 700T series used in the mid-2000s on 2-MW turbines. They 
are 690-V electric motors with a hybrid planetary and worm gear design that results in a 1:1100 gear ratio. According to the manufacturer's specifications, they weigh $190 \mathrm{~kg}$ according to manufacturer specifications.

\subsubsection{Mass Property Calculations}

The friction plate surface width is assumed to be $1 / 10^{\text {th }}$ the tower top diameter, and the friction plate thickness is assumed to be $1 / 1,000^{\text {th }}$ the rotor diameter. These ratios resulted in reasonable agreement with known turbine specifications; however, the variability in tower top diameter, mass, and friction or rolling element bearings results in a range of observed masses for this component. The mass and geometrical properties are calculated according to Eq. 2.63:

$$
V_{\text {yaw }}=\frac{\pi D_{T W}^{2}}{10} \frac{D_{r}}{1000}
$$

where $V_{\text {yaw }}$ gives the friction plate volume, and the mass is simply $M_{\text {yaw }}=\rho V_{\text {yaw }}$ where $\rho$ is the density of steel, taken to be 8,000 $\mathrm{kg} / \mathrm{m}^{3}$ in this case.

It is recommended that the user prescribes the number of yaw motors; however, if the user does not specify the number of yaw motors, it is calculated based on rotor diameter according to the following rough trends observed in industry:

$$
N_{\text {motors }}= \begin{cases}4 & \text { if rotor diameter is }<90 \mathrm{~m} \\ 6 & \text { if rotor diameter is }<120 \mathrm{~m} \\ 8 & \text { if rotor diameter is }>120 \mathrm{~m}\end{cases}
$$

The CM of the yaw system is assumed to be centered on the tower axis, and located below the bedplate, such that:

$$
\left[\begin{array}{ll}
C M_{\text {yaw }}^{x} & =0 \\
C M_{\text {yaw }}^{y} & =0 \\
C M_{\text {yaw }}^{z} & =-H_{\text {bedplate }}
\end{array}\right]
$$

\subsection{Bedplate}

The bedplate module is designed as two distinct front and rear components. The rear frame is modeled as two parallel steel I-beams and the front frame is modeled as two parallel ductile cast iron I-beams. The geometrical and mass properties of the bedplate sections are determined by finding I-beam dimensions that meet tip deflection and stress constraints. The rear bedplate is modeled with static loading from drivetrain and nacelle components. The front bedplate is modeled with static nacelle component loads as well as gravity loads from the rotor and aerodynamic overturning moments. The location of up-tower components is taken from the CM outputs of all other component models. The yaw system is not included in the sizing of the bedplate structure, because its loads are passed directly to the tower. The weight of the gearbox affects the sizing of the front or back sections depending on its location, which can be specified by the user as upwind or downwind from the tower center.

This approach considers only ultimate strength and maximum deflection; however, the deflection and stress constraints are set empirically. The maximum stress is considered as $1 / 7^{\text {th }}$ of the yield stress. The bedplate deflection limit is set as length/1500. The maximum overturning moment is included; however, other aerodynamic loads from the rotor or yaw-induced loads are not considered.

The I-beams are initialized with a flange thickness of $0.01905 \mathrm{~m}$, a web thickness of $0.0127 \mathrm{~m}$, an overall height of $0.6096 \mathrm{~m}$, and an overall width of $0.3048 \mathrm{~m}$. This is similar to a W24 x 100 I-beam (using English units) and gives good proportions as the beam is sized up. The I-beam is iteratively increased in size until the bending stress at the beam root satisfies the stress constraint, and the tip deflection is less than a deflection constraint. After each iteration, the I-beam web and flange thickness is increased by $2 \mathrm{~mm}$, and the overall height and width are increased by $6 \mathrm{~mm}$ until the stress and deflection constraints are satisfied. Table 3 summarizes the mass and geometrical properties.

The deflection of the bedplate is calculated using Euler-Bernoulli beam bending equations found in standard mechanical engineering design books (Shigley et al. 2003). The tip deflection, $T D$, is calculated for a cantilevered beam of length $L$ with a load $F$ applied at an intermediate location $x$ according to Eq. 2.66: 
Table 3. I-Beam Properties

\begin{tabular}{lc}
\hline Parameter & Value \\
\hline Front Length & Upwind Bearing CM $+($ Upwind Bearing Facewidth) $/ 2$ \\
Rear Length (Up-tower Transformer) & Transformer CM $\times 1.1$ \\
Rear Length (Down-tower Transformer) & Generator CM $\times 1.47-$ Tower Top Diameter $/ 2$ \\
$E_{\text {cast }}$ & $169 \mathrm{GPa}$ \\
$E_{\text {steel }}$ & $210 \mathrm{GPa}$ \\
Front Max Stress Constraint & $33.3 \mathrm{MPa}$ \\
Rear Max Stress Constraint & $103.3 \mathrm{MPa}$ \\
Max Deflection Constraint & Length $/ 1,000 \mathrm{~m}$ \\
Cast Density & $7,100 \mathrm{~kg} / \mathrm{m}^{3}$ \\
Steel Density & $7,800 \mathrm{~kg} / \mathrm{m}^{3}$ \\
\hline
\end{tabular}

$$
T D=\frac{F x^{2}(3 L-x)}{6 E I}
$$

where $E$ is the modulus of elasticity for the beam material and $I$ is the moment of inertia about the y-axis for the I-beam. For the rear steel frame, $E=210 \mathrm{GPa}$, and for the front cast iron frame, $E=169 \mathrm{GPa}$.

The deflection caused by the distributed weight $w$ of the I-beam support structure is also calculated according to Eq. 2.67:

$$
D=\frac{w L^{4}}{8 E I}
$$

The deflection caused by an applied moment at the end of a cantilevered beam, which in this case is the rotor overturning moment, is given by:

$$
D=\frac{M_{r} L^{2}}{2 E I}
$$

where $M_{r}$ is the applied rotor overturning moment.

The total tip deflection is calculated by superimposing the tip deflection from each component, the self-weight of the bedplate, and the rotor moment deflection for the front bedplate. The loads are all divided by 2 to reflect the equal weight distribution between the I-beams.

The bending moment about the y-axis at the bedplate root is found for each component as $M=P x$, and the bending moment caused by the distributed beam weight is found from $M=w L^{2} / 2$. Bending moments are summed for the front and rear separately, and the rotor bending moment is added to the front bedplate tally. The maximum bending stress on the bedplate root is then calculated by:

$$
\sigma=\frac{M_{\text {total }} h}{I}
$$

where $M_{\text {total }}$ is the total bending moment, and $h$ is the distance above the neutral axis, which is half the I-beam height at the point of the maximum stress.

\subsubsection{Mass Property Analysis}

Total bedplate mass includes supports and connections that are not included in the parallel I-beam mass calculation. As found in industry data, the bedplates of smaller turbines have a larger percentage of extraneous mass. To fit the observations made in industry bedplate sizing to the model, a mass multiplier is included, which scales to rotor diameter according to the relationship $1.1+5 E 13 \times D_{r}^{-8.0}$, where $D_{r}$ is expressed in meters.

The $\mathrm{CM}$ of the bedplate is determined from the combined mass and length of the bedplate sections as described by Eq. 2.70: 


$$
\begin{array}{ll}
C M_{\text {bedplate }}^{x} & =\frac{\left(m_{\text {rear }} \times L_{\text {rear }} / 2\right)-\left(m_{\text {front }} \times L_{\text {front }} / 2\right)}{m_{\text {rear }}+m_{\text {front }}} \\
C M_{\text {bedplate }}^{y} & =0.0 \\
C M_{\text {bedplate }}^{z} & =-\frac{h_{\text {bedplate }}}{2}
\end{array}
$$

\subsection{Additional Nacelle Components}

Other nacelle components including the generator, transformer, and other auxiliary parts are defined according to DriveWPACT. For the purposes of the DriveSE model, the locations of each one of these components is important to the sizing of the bedplate and the system-level mass-center and inertia analysis; therefore, a more rigorous, size-based mass-center analysis is included in DriveSE for all other components to address these concerns. Because of constraints on scope, however, the existing mass moment of inertia calculations from DriveWPACT were used for all DriveSE components. The scaling relationships defining component masses can be found in the DriveWPACT model documentation (DriveWPACT 2013).

Much like the other CM calculations given previously, it is assumed that the components in this section are distributed uniformly throughout their lengths. When calculating the mass center of the parked rotor-nacelle assembly (RNA), the bedplate CM is assumed to be above the tower top center to avoid the circular dependency problems that arise when the bedplate is considered. An updated bedplate $\mathrm{CM}$ is calculated in the next section.

\subsubsection{Mass Property Calculations}

Changes to DriveWPACT for the other drivetrain components, including generator coupling and generator models, incorporate the lengths defined by DriveSE to define the component CMs relative to each other, rather than those based on scaling relationships. The high-speed side, which includes the mechanical break and HSS, has a CM location in the x-, y-and z-axis defined by Eq. 2.71. The length of the high-speed side is an optional user input, which, if left blank, defaults to $\frac{0.5+D_{r}}{127}$. This formula is derived from an approximate scaling relationship observed for the HSS length:

$$
\left[\begin{array}{ll}
C M_{h s}^{x} & =C M_{g b x}^{x}+\frac{L_{g b x}}{2}+\frac{L_{h s}}{2} \\
C M_{h s}^{y} & =C M_{g b x}^{y} \\
C M_{h s}^{z} & =C M_{g b x}^{z}+H_{g b x} \times 0.2
\end{array}\right]
$$

The $\mathrm{Z}$ position of the HSS is defined as approximately $20 \%$ of the gearbox height above the gearbox CM. This is based on a rough approximation of existing drivetrain topology, but because of the low mass of the high-speed side, it does not have a significant impact on overall CM definitions. Using the same assumptions with respect to drivetrain topology, the mass center of the generator is defined relative to the high-speed side according to Eq. 2.72.

$$
\left[\begin{array}{ll}
C M_{\text {gen }}^{x} & =C M_{h s}^{x}+\frac{L_{h s}}{2}+\frac{L_{\text {gen }}}{2} \\
C M_{\text {gen }}^{y} & =C M_{h s}^{y} \\
C M_{\text {gen }}^{z} & =C M_{h s}^{z}
\end{array}\right]
$$

No transformer mass was included in DriveWPACT, but the option of an up-tower transformer and associated mass is included in DriveSE. The mass of the up-tower transformer is calculated based on an extrapolation of known weights for ABB RESIBLOC cast-resin dry-type transformers operating at 24/36 kV (ABB 2011). Dry transformers such as these are typically used in up-tower applications instead of wet oil-cooled transformers. The mass of these transformers exhibits a nearly perfect linear correlation to their power rating, allowing for accurate interpolation or extrapolation to other transformer sizes. The scaling relationship between transformer mass and transformer power, $P_{t}(\mathrm{kVA})$, is:

$$
m_{t}=2.4445 P_{t}+1599
$$

If the transformer is up-tower, the model chooses its location such that the total RNA CM is within the perimeter of the tower bottom. This design option uses the transformer's relatively large mass to reduce the stationary moments caused by the RNA CM. Here, it is assumed that the bottom diameter of the tower is defined as approximately TowerTopDiameter $\times 1.7$, which is taken from an average of diameter ratios found in industry. The mass center of the transformer is calculated according to Eq. 2.74: 


$$
\left[\begin{array}{ll}
C M_{t}^{x}(\text { approach } 1) & =C M_{g e n}^{x}+\frac{L_{G E}}{2}+\frac{L_{T F}}{2} \\
C M_{t}^{x}(\text { approach } 2) & =\frac{D_{T B}}{2} \times\left(m_{R N A}+m_{t}\right)-\left(m_{R N A} \times C M_{R N A}^{x}\right) \\
m_{t} & =C M_{G E}^{y} \\
C M_{t}^{y} & =C M_{g b x}^{z}
\end{array}\right]
$$

If the RNA CM is located outside the perimeter of the tower bottom, the first equation in Eq. 2.74 (approach 1) is used. The second equation in Eq. 2.74 (approach 2) places the transformer at an excessively large distance from the tower top center (defined by $3 \times G e n_{x}$ ). An error is displayed and the first equation is used instead. If the error is displayed and the RNA CM is of concern, users can ensure that it is acceptable by manually moving the gearbox downwind from the tower top center. 


\section{Model Verification Against Higher Fidelity Models}

Verification of the model against higher fidelity analysis was performed using solid models created from three differently rated drivetrains. From dimensions and drawings obtained from a reference 750-kW turbine, WindPACT 1.5-MW (DriveWPACT 2013), and 5-MW turbine, solid parts were created for the hub, main shaft, and bedplate of each assembly. When detailed drawings were not available, such as the 5-MW reference turbine, parts were approximated via scaling available models based on smaller turbines. Appendix A contains tables that detail model inputs to DriveSE for these three turbines.

Loads from design load case simulations carried out in FAST (Jonkman et al. 2011) were compiled for each machine, and a combination of the largest magnitude loads were applied to the solid models in ANSYS. FEA studies were performed using ANSYS to examine the static response of each component to their respective extreme loads. Stress and deflection results were recorded and compared to expected values. Data from the solid models and FEA simulations served three primary functions:

- Verify assumptions made in DriveSE based on the location of largest stress concentrations and deflections

- Yield quantitative insight into von Mises stress magnitudes and factors of safety for each modeled component at extreme design load case conditions

- Calculate solid model mass and dimensions for comparison to DriveSE model output sizing.

Figure 10 shows an example FEA process for the $750-\mathrm{kW}$ main shaft.

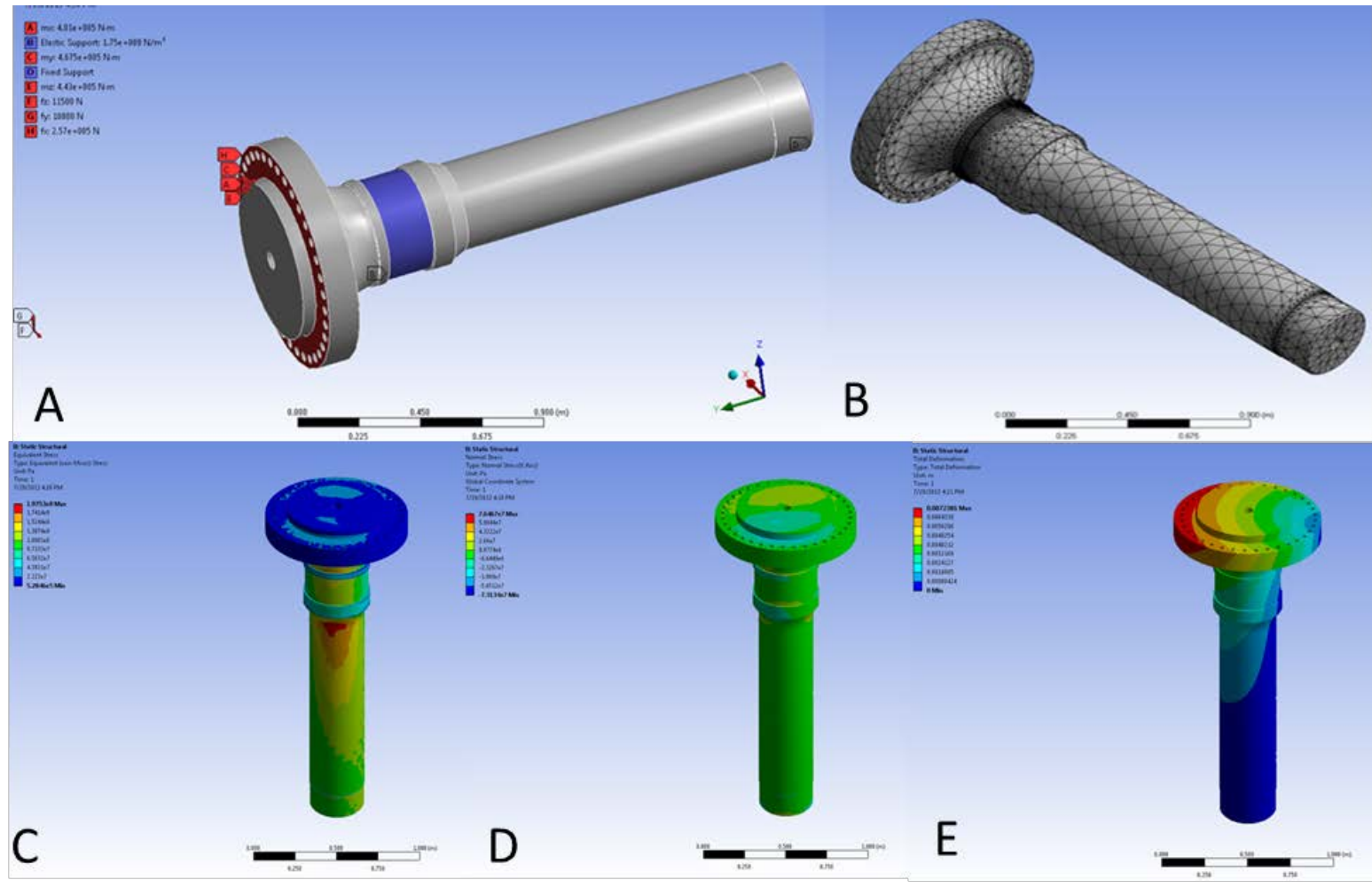

Figure 10. (A) Loads and constraints applied to the 750-kW LSS; (B) the mesh used in the analysis; and the plots of (C) von Mises stress, (D) shear stress, and (E) deformation

\subsection{Main Shaft Analysis}

Results of the solid modeling process include component geometries and masses that were used to compare against DriveSE outputs. 
FEAs were performed on the main shafts of the largest and smallest reference turbines to verify key assumptions in the main shaft model. The 750-kW reference is a three-point suspension configuration; the 5-MW reference is a four-point suspension. The selected extreme rotor loads, found in Table 4, were applied as remote loads to the hub center and projected onto the flange connection of the shaft.

Table 4. Loads Applied to Main Shaft Components, in Global Nacelle Coordinate System

\begin{tabular}{ccccccc}
\hline Turbine & $\mathrm{Fx}(k N)$ & $\mathrm{Fy}(k N)$ & $\mathrm{Fz}(k N)$ & $\mathrm{Mx}(k N m)$ & $\mathrm{My}(k N m)$ & $\mathrm{Mz}(k N m)$ \\
\hline $750 \mathrm{~kW}$ & 257.0 & 1.1 & -11.5 & 401.0 & -467.5 & -443.0 \\
$5 \mathrm{MW}$ & $1,530.0$ & $1,770.0$ & $-1,750.0$ & $7,840.0$ & $-14,700.0$ & $-12,900.0$ \\
\hline
\end{tabular}

Bearings were simulated using cylindrical supports with elasticities typical of an upwind CARB bearing or downwind SRB bearing found on manufacturer data sheets. At the downwind end of the shafts, at the shrink-disk connection to the gearbox, several nodes were fixed to prevent rigid body motion. The artificially abnormal stress concentrations arising from full fixity was ignored for the purpose of analysis. Results of the FEA analysis are shown in Table 5.

Table 5. Resulting Maximum Stress Concentrations and Deflections at the Location of the Bearings

\begin{tabular}{ccc}
\hline Turbine & von Mises Stress $(\mathrm{MPa})$ & Bearing Deflection $(\mathrm{mm})$ \\
\hline $750 \mathrm{~kW}$ & 197.5 & 3.8 \\
$5 \mathrm{MW}$ & 565.6 & 3.8 \\
\hline
\end{tabular}

The results of these studies show that the largest stress concentrations, which are used to size the main shaft in the DriveSE model, occur at the upwind side of the main bearings. It is important to note that this assumption is verified for both three-point ant four-point configurations. In addition, results show main shaft deflections at the locations of the bearings that are within tolerance for the specified main bearings. As a final check, the magnitudes of the von Mises stresses do not exceed the yield strength of the shaft material, which verifies that the solid models themselves do not fail and yields insight into the safety factors that original equipment manufacturers may have used on the main shaft.

\subsection{Bedplate Analysis}

Methods used to size the bedplates were validated by testing the simple I-beam geometries created by the DriveSE bedplate model. Loads applied to the front cast iron I-beam included the weights of the main bearing(s), the main shaft weight, and rotor overhang moments and forces. Nodes at the downwind face of the front I-beam were fixed. To match the assumptions made in the model, component weights were applied at the CM of each component, and all loads except for the self-weight of the beams were halved to simulate the even distribution across two parallel beams. Finally, mesh resolution studies were performed on the top of the fixtures, where bending stresses are greatest, until the stress values converged.

Figure 11 shows the basic I-beam assembly in SolidWorks, with split lines at the locations of each component load. The FEA results were compared to the calculated DriveSE loads, as shown in Table 6 . The results of this study closely agree with the stresses and deflections calculated by DriveSE. From this, it can be concluded that the equations used to size a bedplate are accurate for this simplified geometry.

Table 6. Bending Stress and Tip Deflection Compared for Rear Beams

\begin{tabular}{lcc}
\hline & Bending Stress $(\mathrm{MPa})$ & Tip Deflection $(\mathrm{mm})$ \\
\hline DriveSE & 50.8 & 2.83 \\
FEA & 53.5 & 2.88 \\
\hline
\end{tabular}

A similar study was performed for the front portion of the bedplate. Because of the larger cross-sectional area and shorter length of this beam, the analysis yielded results that were slightly different from those predicted by DriveSE. Table 7 compares the FEA 


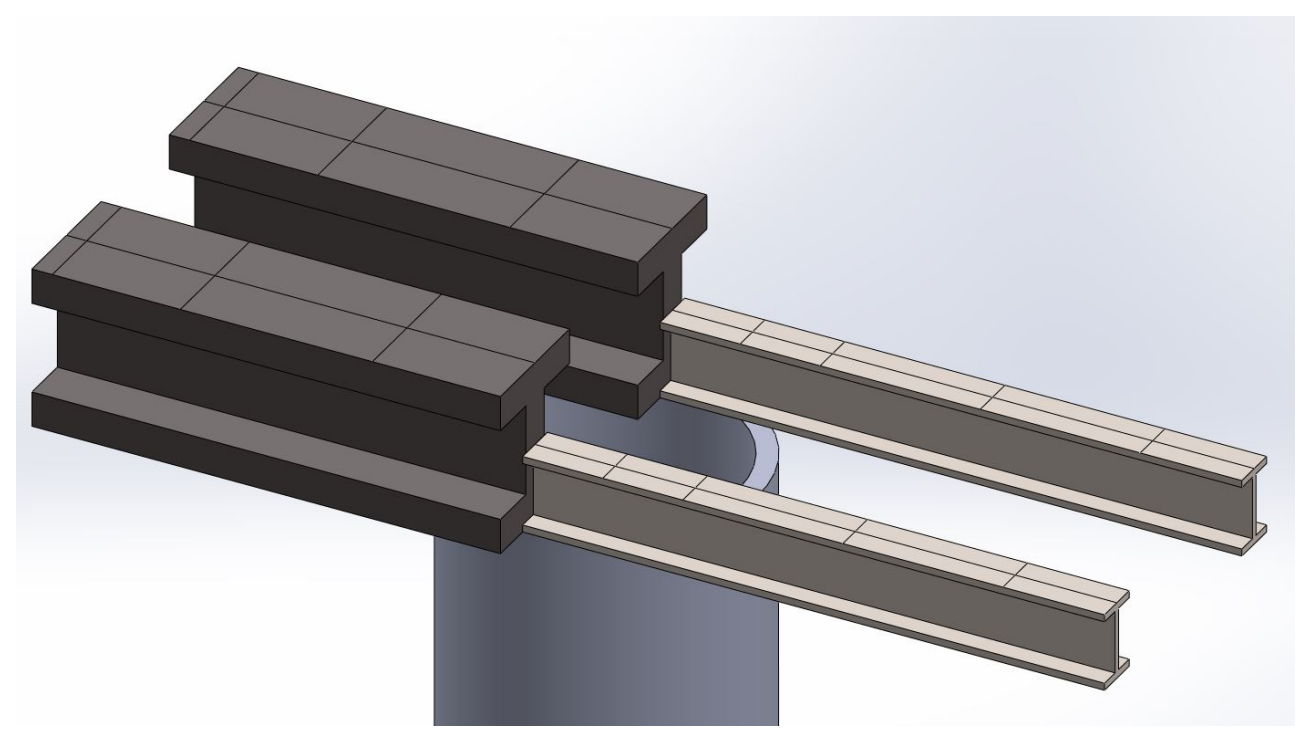

Figure 11. DriveSE basic I-beam assembly with large front cast iron beam and long rear steel piece. Note the split lines across the top faces of I-beams at the component locations.

results of one such front I-beam study to the stress and deflection predictions made by DriveSE. The difference here points to a slight discrepancy between the model results and reality. The front bedplate algorithm uses Euler-Bernoulli beam theory, which neglects shear effects that become more prevalent in shorter beams. As a result, DriveSE underpredicts critical stresses on the front I-beam assembly; however, because this model already embodies unrealistic assumptions by using simple I-beam geometry, the discrepancy (given the other limitations of the model) was deemed acceptable.

Table 7. Maximum Bending Stress and Tip Deflection Compared for Front Beams

\begin{tabular}{lcc}
\hline & Bending Stress $(\mathrm{MPa})$ & Tip Deflection $(\mathrm{mm})$ \\
\hline DriveSE & 39.1 & 3.55 \\
FEA & 41.1 & 3.39 \\
\hline
\end{tabular}

The results of these studies show that under the given assumptions, the DriveSE sizing model accurately predicts maximum stress and deflection locations and magnitudes, values that are used to determine component sizes. As more detailed geometries are located, high-fidelity FEA verification steps may be taken to show the similarities between the assumed I-beam geometry and real-world bedplate configurations. 


\section{Model Validation and Results}

The model was tested using the inputs from three machines of varying size, and compared to the known data on component dimensions and weight. The studied reference turbines are GRC 750-kW (Link et al. 2011), WindPACT 1.5-MW (DriveWPACT 2013), and NREL 5-MW reference (Dykes et al. 2014b) turbines. The model inputs for all three turbines are all included in Appendix A. The 750-kW turbine is a three-point suspension drivetrain design with a single main bearing; the larger turbines both incorporate two main bearings into their designs. Also note that the extreme loads inputs for each machine were taken from the outputs of previous aeroelastic simulations, and are not guaranteed in their accuracy.

\subsection{Hub}

DriveSE hub mass outputs are listed in Table 8 and compared to the approximated masses found from the three-dimensional modeling efforts. DriveSE models the hub as a hollow cylinder with holes for the blade root and main shaft connection; hubs in the industry are more spherical in nature. For this reason, the outer diameters are not compared.

Table 8. Comparison of Hub Model Outputs to FE Model Masses

\begin{tabular}{l|lll} 
& \multicolumn{3}{|c}{ Hub Mass } \\
& FEA $(\mathrm{kg})$ & DriveSE $(\mathrm{kg})$ & Difference (\%) \\
\hline 5 MW & $56,424.2$ & $60,775.5$ & 7.7 \\
$1.5 \mathrm{MW}$ & $19,560.3$ & $18,762.6$ & -4.1 \\
$750 \mathrm{~kW}$ & $2,604.0$ & $2,751.7$ & 6.1
\end{tabular}

The hub results from all three machines show relatively close agreement to the predicted hub masses. Note that the blade root diameter for the 750-kW turbine was specified as a known input; the blade root diameters for the other two turbines were left to DriveSE's internal scaling arguments. The outputs from DriveSE are plotted against the variety of industry data available on hub masses, as shown in Figure 12. From these results, it can be concluded that the hub model predicts hub mass for a variety of machine sizes with accuracy within $+/-10 \%$.

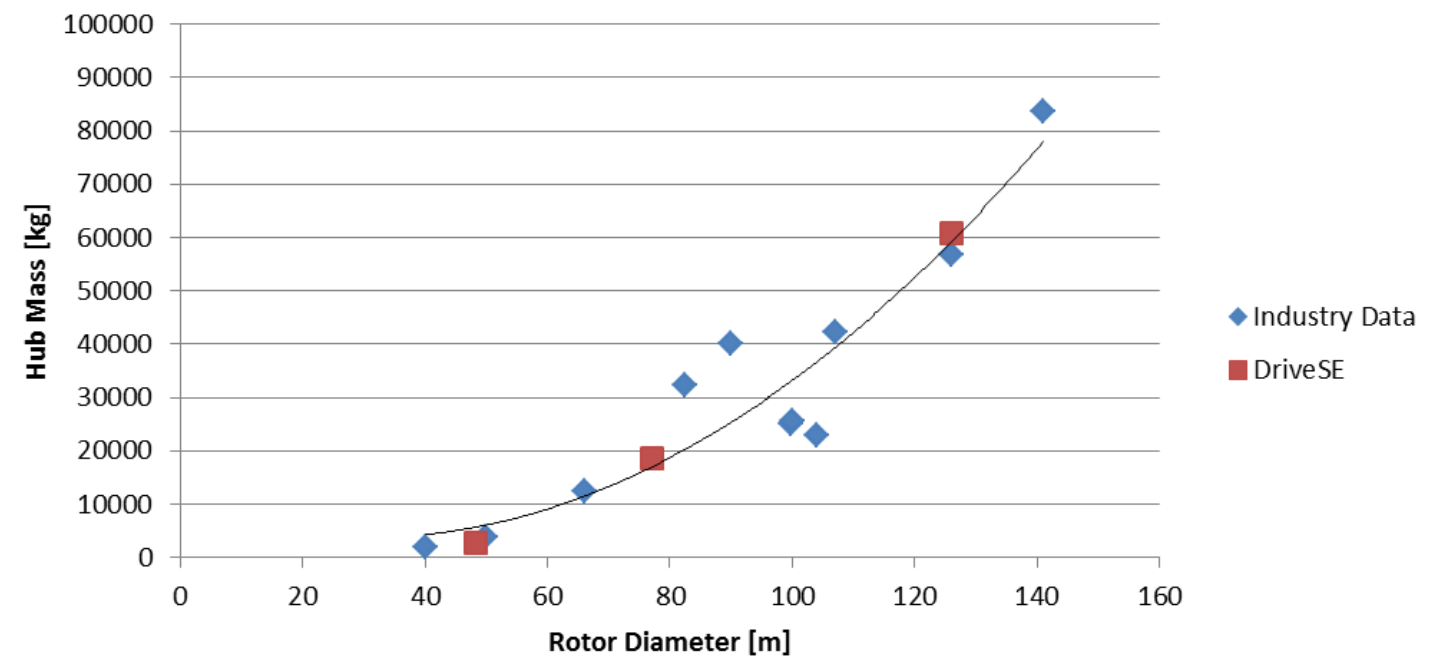

Figure 12. Industry trend and model results: hub mass compared to turbine nameplate rating 


\subsection{Main Shaft and Bearings}

The main shaft and main bearing sizing models were validated using the dimensions and weights from three reference machine solid models. In the first simulation, the fatigue option was turned off, and only maximum stress and deflection were considered. The output dimensions for the three simulations are shown in Table 9 and compared to the known industry values.

Table 9. Comparison of Nonfatigue Main Shaft Model Output and Solid Mass Dimensions

\begin{tabular}{l|lll|lll|lll} 
& \multicolumn{3}{|c}{ Length $(\mathrm{m})$} & \multicolumn{3}{c}{ Upwind Diameter $(\mathrm{m})$} & \multicolumn{3}{c}{ Downwind Diameter $(\mathrm{m})$} \\
& FEA & DriveSE & Difference $(\%)$ & FEA & DriveSE & Difference $(\%)$ & FEA & DriveSE & Difference $(\%)$ \\
\hline $5 \mathrm{MW}$ & 3.11 & 3.16 & 1.5 & 1.00 & 1.06 & 6.0 & 0.72 & 0.71 & -1.39 \\
$1.5 \mathrm{MW}$ & 2.54 & 2.1 & -17.2 & 0.60 & 0.60 & 0.0 & 0.51 & 0.36 & -29.41 \\
$750 \mathrm{~kW}$ & 2.05 & 2.06 & 0.5 & 0.38 & 0.42 & 10.5 & 0.325 & 0.43 & 32.31
\end{tabular}

This table shows that under extreme loading inputs, the model is relatively accurate in predicting the shaft lengths and upwind diameters, but there is some variability in the results for the downwind diameters. Also, the 1.5-MW reference main shaft seems to be larger than the industry trend, as shown in Figure 13, which may explain the discrepancies in these results. After the parameterized fatigue option is turned on, shaft lengths remain the same, but diameters are increased when the model finds that fatigue is the governing load case.

\section{Table 10. Comparison of Fatigue Main Shaft Model Output and FE Model Dimensions}

\begin{tabular}{l|lll|lll} 
& \multicolumn{3}{|c}{ Upwind Diameter (m) } & \multicolumn{3}{c}{ Downwind Diameter (m) } \\
& FEA & DriveSE & Difference (\%) & FEA & DriveSE & Difference (\%) \\
\hline $5 \mathrm{MW}$ & 1.00 & 1.06 & 6.0 & 0.72 & 0.71 & -1.39 \\
$1.5 \mathrm{MW}$ & 0.60 & 0.63 & 5.0 & 0.51 & 0.50 & -1.96 \\
$750 \mathrm{~kW}$ & 0.38 & 0.42 & 10.5 & 0.325 & 0.43 & 32.31
\end{tabular}

Note that under fatigue, the downwind diameter of the 1.5-MW machine is predicted much more accurately than if only extreme loads were used. This lends credibility to the fatigue model as a tool for sizing the main shaft and bearing diameters.

To gauge the accuracy of DriveSE's main shaft mass calculation, the mass results from the fatigue model are compared to the mass results of the nonfatigue model, as in Table 11.

Table 11. Comparison of Fatigue and Nonfatigue Models to the FE Model Mass

\begin{tabular}{l|lll|lll} 
& \multicolumn{3}{|c}{ Extreme Loads Mass (kg) } & \multicolumn{3}{c}{ Fatigue Mass (kg) } \\
& FEA & DriveSE & Difference (\%) & FEA & DriveSE & Difference (\%) \\
\hline $5 \mathrm{MW}$ & 21,044 & 16,898 & -19.7 & 21,044 & 16,898 & -19.7 \\
$1.5 \mathrm{MW}$ & 6,318 & 3,420 & -45.9 & 6,318 & 4,681 & -25.9 \\
$750 \mathrm{~kW}$ & 2,000 & 2,556 & 27.8 & 2,000 & 2,556 & 27.8
\end{tabular}

The mass results show a significant amount of variability between the predicted industry mass and DriveSE output. From the three available data points, it can be hypothesized that DriveSE may tend to overpredict the mass of three-point suspension main shafts and underpredict the main shafts of four-point suspension drivetrains. The diameter predictions are relatively accurate for all turbine configurations under the provided loads. The discrepancies may be a consequence of the fact that flange and shrink gearbox-attachment sizing varies among wind turbine original equipment manufacturers. The increased accuracy in the 1.5-MW sizing after fatigue analysis is performed results in a better estimate for the 1.5-MW mass, but still with significant error. Reasons for this discrepancy may include errors in the loads data or inaccuracies in the assumptions made in the stress calculations.

Figure 13 plots the output masses for the fatigue and extreme load model against each machine's nameplate rating and compares the results to other known industry data. This trend shows that, despite mass errors on the order of $25 \%$ from individual shaft masses, the model reasonably captures the industry trend of main shaft sizes, especially for smaller turbines.

The general trend of industry data and main shaft sizing shown in Figure 13 proves that the model is a suitable starting point for sizing the main shaft using expected load data. 


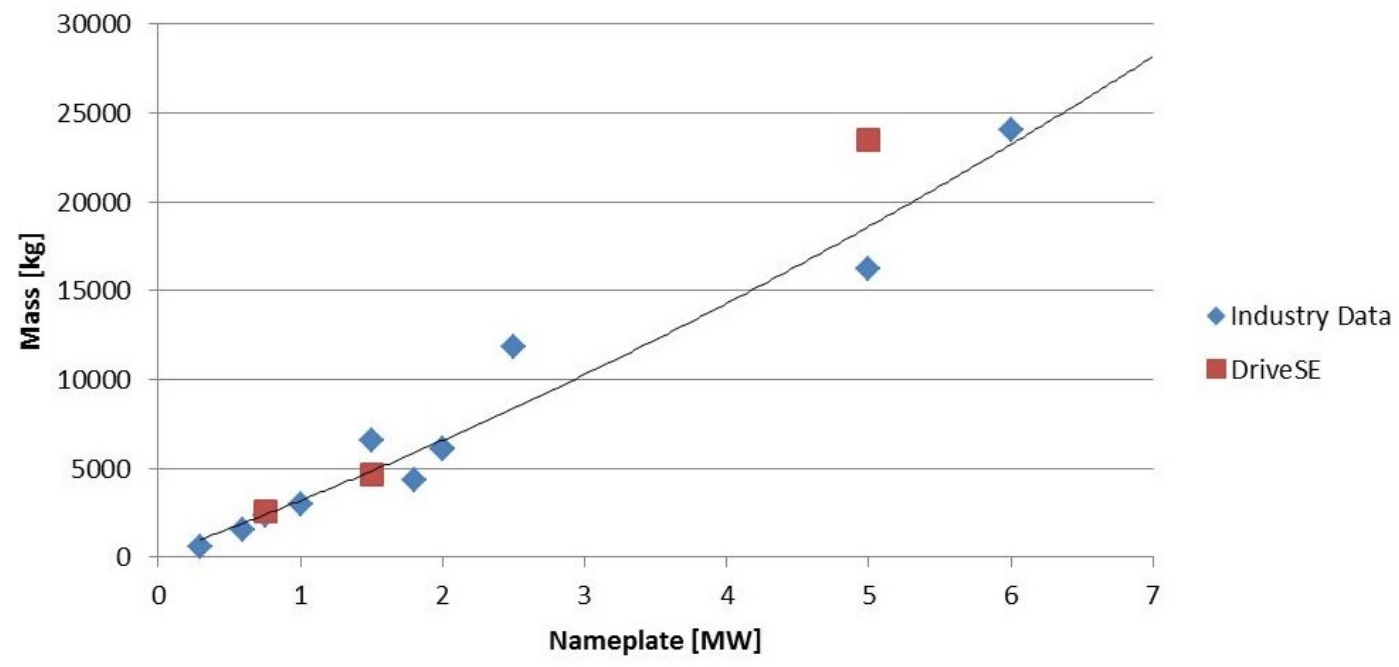

Figure 13. Industry trend and model results: main shaft mass compared to turbine rating

\subsection{Gearbox Stage Ratio and Weight}

The calculated speed ratios for each gear stage of the GRC 750-kW turbine is compared to the design values, as shown in Table 12. These two gearboxes have two of the most popular gearbox configurations: epp for gearboxes less than 3 MW and eep for gearboxes equal to and more than $3 \mathrm{MW}$. The calculated optimal speed ratios match the designed values for the epp gearbox.

Table 12. Gearbox Speed Ratio per Stage of the GRC 750-kW Gearbox

\begin{tabular}{lccc}
\hline & Stage 1 & Stage 2 & Stage 3 \\
\hline \hline Type & Planetary & Parallel & Parallel \\
Design & 5.71 & 3.57 & 4.00 \\
Model & 5.77 & 3.32 & 4.26 \\
Difference & $1.06 \%$ & $7.00 \%$ & $6.50 \%$ \\
\hline
\end{tabular}

The computed gearbox weights are compared to the design values for a variety of gearboxes ranging from $750 \mathrm{~kW}$ to $5 \mathrm{MW}$, as shown in Figure 14. When input torque is larger than $1,386.5 \times 10^{3} \mathrm{~kg}(3 \mathrm{MW})$, the gearboxes have the eep configuration instead of the epp configuration for the gearboxes with smaller input torque. The agreement between the model predictions and actual values is excellent. The agreement between the model predictions and actual values is good in general using the model with the optimized speed ratios for the gearboxes with the eep configuration. The predicted gearbox masses for large turbines are lower than those found in the industry data, which suggests that there may be room for gearbox weight reductions for large turbines in the market. Furthermore, the gearbox design for large turbines is affected by the manufacturing capacity, usage of the existing machining tools for small- to medium-size gearboxes, consideration of the ease of disassembly of high-speed gearbox stages, and fitting catalog bearings in planetary gears, among other factors. These factors also contribute to the differences seen in the model and industry data. 


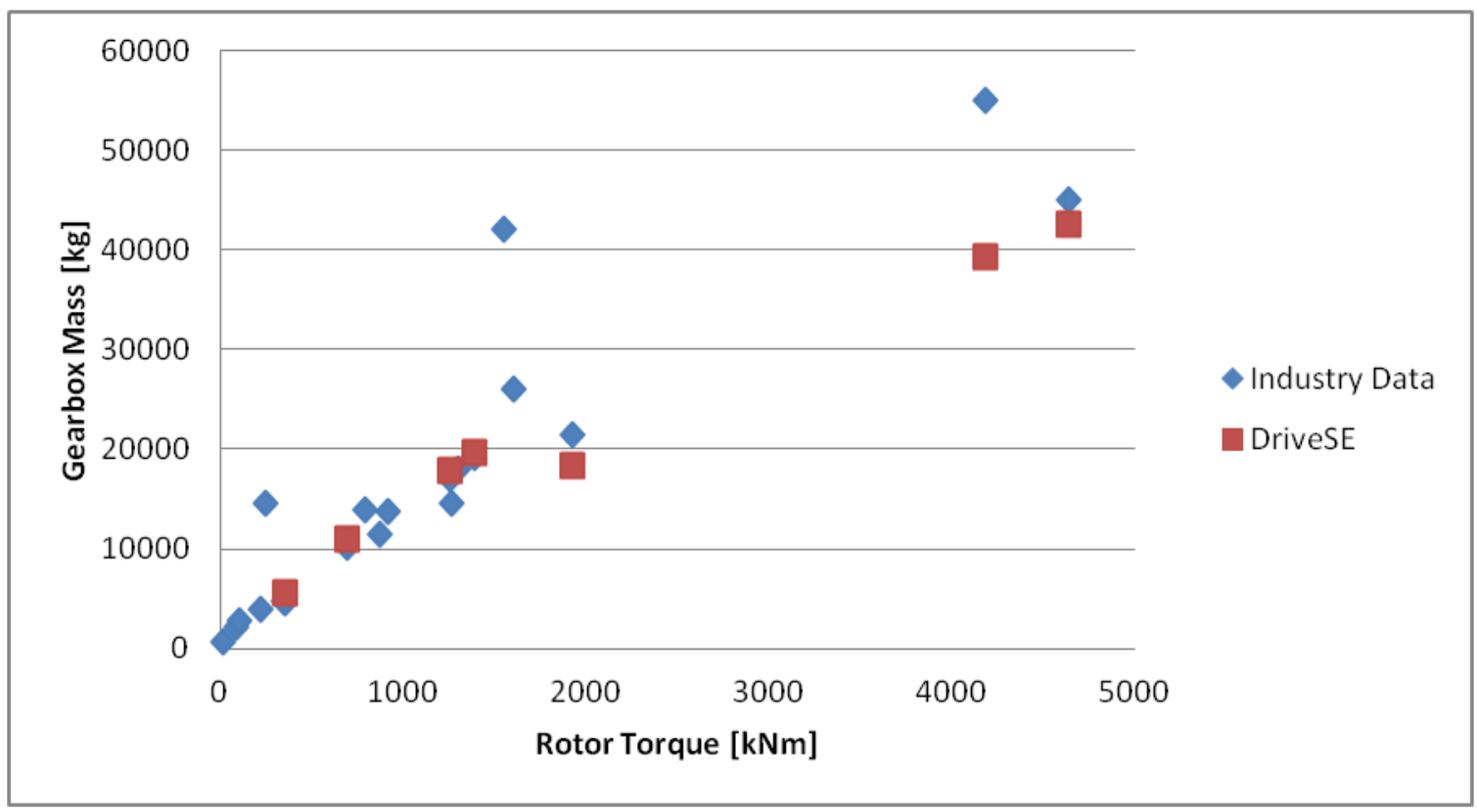

Figure 14. Industry trend and model results: gearbox weight compared to rated torque

\subsection{Bedplate}

The bedplate sizing model was tested using loads data from all three reference turbines. Comparisons between solid model mass and predicted mass were made for the 750-kW and 1.5-MW machines because the 5-MW reference turbine bedplate characteristics are not defined. Table 13 shows the results of this analysis.

Table 13. Comparison of Bedplate Model Outputs to FE Model Masses

\begin{tabular}{l|lll} 
& \multicolumn{3}{|c}{ Bedplate Mass $(\mathrm{kg})$} \\
& FEA & DriveSE & Difference $(\%)$ \\
\hline $1.5 \mathrm{MW}$ & 10140 & 9985 & -1.5 \\
$750 \mathrm{~kW}$ & 5520 & 5036 & -8.8
\end{tabular}

The data from the 1.5-MW turbine show strong agreement between the model and industry sizing. This is partially because the actual 1.5-MW reference machine's bedplate very closely resembles the two parallel I-beams configuration used to size the bedplate in the DriveSE model. The mass difference shown in the 750-kW turbine is partially because nonstructural portions of the bedplate comprise a more significant percentage of the smaller bedplate's mass.

To determine the effects of an up-tower transformer on the bedplate model, the 1.5-MW turbine inputs were changed to include a transformer. Results of this simulation are given in Table 14.

Table 14. Effects of Transformer Location on Bedplate Dimensions and Mass for the 1.5-MW Turbine

\begin{tabular}{l|ll} 
& Bedplate Length (m) & Bedplate Mass (kg) \\
\hline Down-Tower Transformer & 5.0 & 9,985 \\
Up-Tower Transformer & 7.2 & 11,044
\end{tabular}

As expected, in an up-tower transformer configuration, the bedplate is significantly longer than that with a down-tower transformer. This added length contributes to a more massive bedplate, but not proportionately so, because the rear steel piece carries less load than the front cast-iron one.

Figure 15 graphs the output mass of the 5-MW, 1.5-MW, and 750-kW machines against the few known industry masses that are 
available. This comparison indicates that the model may overpredict bedplate masses in larger-scale turbines and underpredict turbines at a smaller scale. At larger scales, bedplate designs tend to diverge from the assumed I-beam configuration to conserve costs. In addition to the reasons listed previously for the discrepancies, such as the underprediction of smaller bedplates, there may be a consequence of error in input load data or invalid assumptions about component location within the bedplate model. Overall, the accuracy of these results validates the bedplate model as an appropriate tool to determine first-order bedplate sizing from loads inputs.

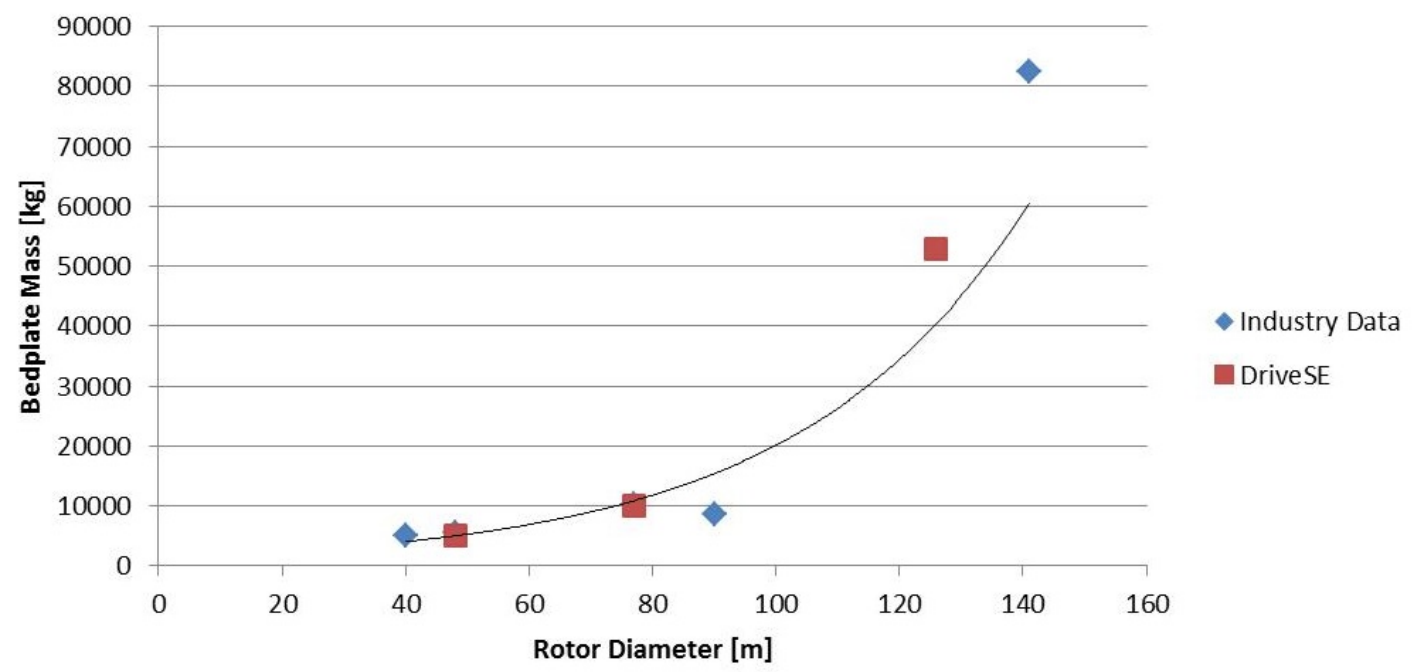

Figure 15. Industry trend and model results: bedplate mass compared to rotor diameter

\subsection{Nacelle Mass}

After the mass of each individual component in the drivetrain is determined, the additional nacelle components are sized based on scaling arguments and added into a total nacelle mass. A diagram showing the DriveSE model's ability to predict total nacelle mass is shown in Figure 16.

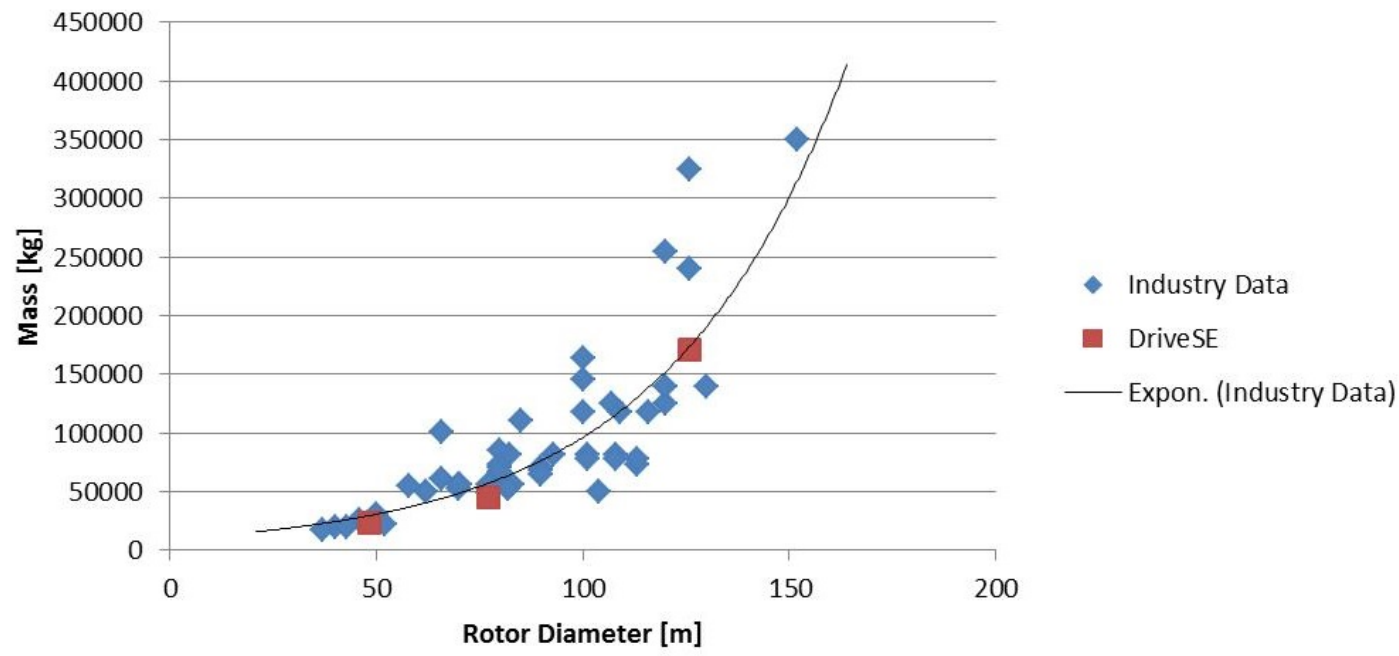

Figure 16. Industry trend and model results: nacelle mass compared to rotor diameter 
Total nacelle mass depends on the topology of a drivetrain. Because of the variety of configuration options available in design, a large amount of scatter is observed in reported industry data. Despite the large range of nacelle masses found in industry, the three-point and four-point DriveSE models both closely follow a curve fit of the data. The trend shown in Figure 16 validates the nacelle-level design assumptions made in DriveSE; all three model masses are slightly lighter than the industry trend, but well within the distribution of the data. 


\section{Conclusions}

This report describes a new model set for sizing major wind turbine drivetrain components. DriveSE provides physics-based methods for sizing the main load-bearing components of a wind turbine drivetrain, including the main shaft and bearings, gearbox, and bedplate. In addition, industry data were used to develop parametric sizing models for the hub, yaw system, and transformer. For the hub system and nacelle, DriveSE uses DriveWPACT methods for sizing, new CM methods were created to properly determine the overall mass properties of each component as well as the entire hub and drivetrain assembly. The output of the model set can be used in an overall wind turbine design, as well as an overall system analysis that includes cost models for the turbine and overall wind power plant.

In addition to presenting the theory and main equations for each component model, the report provides verification and validation results. The physics-based models were verified against finite-element models, and all component models were validated to the extent possible against industry data for actual component dimensions and masses. The results show that the models accurately represent actual component dimensions and masses to a first order; however, because of the simplicity of the physics in the models, differences remain between the model results and real-world data. These discrepancies motivate future work on development of all the models.

There are several potential directions for improving the different component models as well as the overall set of models in DriveSE:

- Advanced Gearbox Rating Model: A new capability of designing gearbox components will be added to DriveSE. This allows bending, pitting, and scuffing rating analysis to be performed for individual components. This advanced approach (refer to Figure 8) would give detailed gear and bearing sizes, and improve cost estimates. The main shaft configuration and shaft length affect the gearbox design by influencing the nontorque loads transmitted into the gearboxes.

- Generator Physical Model: Generator sizes and masses will be determined from electrical and physical factors in the system rather than from the scaling arguments that are currently present.

- Hub Physical Model: The current hub model relies on scaling arguments that, although more accurate than similar hub models, are still not based on blade loads. In the future, these scaling relationships will be replaced by constraints on blade root bending moment.

- Design for Fatigue: With the exception of the main shaft and bearings, DriveSE does not consider fatigue in other sizing algorithms. In the next phase, fatigue analysis will be added to the hub and gearbox, which can improve the drivetrain design accuracy.

- Automatic Loads Selection: Current models take a single set of loads inputs from loads simulations and size components based on the assumption that these loads generate maximum stresses and deflections throughout the assembly. In the future, functionality may be added that cycles through all extreme design load case results and sizes different components based on their respective governing load cases.

- Data Collection: Drivetrain information for wind turbines $>3 \mathrm{MW}$ is scarce. This information is valuable for benchmarking the DriveSE models and will be updated as data become available.

- Case Studies: The DriveSE model can be used to investigate the effects of several design parameters for the size of a wind turbine drivetrain.

- Integrated and Direct-Drive Configurations: In addition to the geared drivetrains, integrated and direct-drive configurations will be added to DriveSE. This new capability will allow for the comparison of multiple designs with various drivetrain configurations and the design flexibility for innovative gearboxes. 


\section{References}

ABB (2011). Technical Data for RESIBLOC Cast-Resin Transformers, Tech. Rep. https://library.e.abb.com/public/1 fc89edc078adeecc1257869004cb360/1LDE000075\%20en\%20d ata\%20sheet\%20RESIBLOC.pdf

American Gear Manufacturers Association (AGMA) (2006). ANSI/AGMA 6123 B06(2), Design Manual for Enclosed Epicyclic Gear Drives. Tech. Rep., Alexandria, VA.

AGMA. (2008). ANSI/AGMA 6001-E08: Design and Selection of Components for Enclosed Gear Drives. Tech. Rep., Alexandria, VA.

AGMA (2010). ANSI/AGMA/AWEA 6006-A03: standard for design and specifications of gearboxes for wind turbines, Tech. Rep., AGMA, Alexandria, VA.

Avallone, E.A.; Baumeister, T.; Sadegh, A.M. (2006). Mark's Standard Handbook for Mechanical Engineers. $11^{\text {th }}$ edition. New York: McGraw-Hill Professional.

"Bearing Product Tables." (2014). SKF. Accessed May 28, 2015:

http://www.skf.com/us/products/bearings-units-housings/product-tables/index.html.

Danish Standards Foundation (DSF) (1992). DS 472: Loads and Safety of Wind Turbine Construction, Denmark. $1^{\text {st }}$ edition.

"DriveWPACT." (2013). National Renewable Energy Laboratory. NWTC Information Portal. Accessed May 28, 2015: https://nwtc.nrel.gov/content/drivewpact.

Dudley, D.W. (1984). Handbook of Practical Gear Design, New York: McGraw-Hill Book Company.

Dykes, K.; Resor, B.; Platt, A.; Guo, Y.; Ning, A.; King, R.; Parsons, T.; Petch, D.; Veers, P. (2014). Effect of Tip-Speed Constraints on the Optimized Design of a Wind Turbine. NREL/TP5000-61726. Golden: CO: National Renewable Energy Laboratory, 2014. Accessed May 28, 2015: http:/www.nrel.gov/docs/fy15osti/61726.pdf.

Gundtoft, S. (2009). Wind Turbines, Tech. Rep., Aarhus, Denmark: University College of Aarhus, Accessed May 28, 2015:

http://staff.iha.dk/sgt/Downloads/Turbines\%20May4_2009_1.pdf

Harrison, R.; Jenkins, G. (1993). Cost Modeling of Horizontal Axis Wind Turbines. Energy Technology Support Unit., Tech. Rep. ETSU/W-34-00170-REP, University of Sunderland, School of Environment, Sunderland, UK.

International Electrotechnical Commission (IEC). (1992). 61400-1, Design Requirements for Wind Turbines, Tech. Rep.

IEC (2005). DS 472: Loads and Safety of Wind Turbine Construction, Denmark: Danish Standard Foundation, 1st edn. 
International Standards Organization (ISO). (2012). IEC/FDIS 61400-4: design requirements for wind turbine gearboxes, Tech. Rep.

Jonkman, J.; Sprague, M.; Jonkman, B. (2011). FAST Modular Wind Turbine CAE Tool:

Nonmatching Spatial and Temporal Meshes: Preprint. NREL/CP-2C00-60742. Golden, CO:

National Renewable Energy Laboratory. Accessed October 7, 2014:

http:/www.nrel.gov/docs/fy14osti/60742.pdf.

King, R.; Guo, Y.; Parsons, T.; Dykes, K. (2014). A Systems Engineering Analysis of 3-point and 4-point Wind Turbine Drivetrain Configurations. Forthcoming. Golden, CO: National Renewable Energy Laboratory, 2014.

Link, H.; LaCava, W.; van Dam, J.; McNiff, B.; Sheng, S.; Wallen, R.; McDade, M.; Lambert, S.; Butterfield, S.; Oyague, F. (2011). Gearbox Reliability Collaborative Project Report:

Findings from Phase 1 and Phase 2 Testing. NREL/TP-5000-51885, Golden, CO: National Renewable Energy Laboratory, 2011. Accessed May 28, 2015:

http://www.nrel.gov/docs/fy11osti/51885.pdf.

Ning, A.; Dykes, K. (2014). "Understanding the Benefits and Limitations of Increasing Maximum Rotor Tip Speed for Utility-Scale Wind Turbines.” Journal of Physics: Conference Series 524 (012087).

Norton, R.A. (2014). Machine Design: An Integrated Approach. $5^{\text {th }}$ edition. Prentice Hall.

Schultz, C.D. (2009). The effect of gearbox architecture on wind turbine enclosure siz., American Gear Manufacturers Association (09FTM19).

Shigley, J.; Mischke, K.; Budynas, R. (2003). Mechanical Engineering Design. 7th edition. Boston: McGraw-Hill Science/Engineering/Math. ISBN 9780072921939.

Willis Jr., R.J. (1963). New equations and charts pick off lightest weight gears, Product Engineering, p. 64-75. 


\section{Appendix A: Reference Model Inputs}

The following tables detail the model inputs that define each reference turbine used in the validation. Unless otherwise noted, units are in (meters, kilowatts, newtons, newton-meters, revolutions per minute, and meters per second). Note that rotor masses are zero, because rotor mass is already considered in the loads.

Table 15. 750-kW Reference Inputs

\begin{tabular}{|c|c|}
\hline rotor diameter & 48.2 \\
\hline DrivetrainEfficiency & 0.95 \\
\hline machine rating & 750 \\
\hline rotor torque & $1.5 *($ machine rating $* 1000 /$ DrivetrainEfficiency $) /($ rotor speed $*(\mathrm{pi} / 30))$ \\
\hline rotor thrust & 143000.0 \\
\hline rotor mass & 0.0 \\
\hline rotorRatedRPM & 22.0 \\
\hline rotor bending moment & $495.6 \mathrm{e} 3$ \\
\hline rotor bending moment $\mathrm{x}$ & $401.0 \mathrm{e} 3$ \\
\hline rotor bending moment y & $495.6 \mathrm{e} 3$ \\
\hline rotor bending moment $\mathrm{z}$ & $-443.0 \mathrm{e} 3$ \\
\hline rotor force $\mathrm{x}$ & 143000.0 \\
\hline rotor force $\mathrm{y}$ & -12600.0 \\
\hline rotor force $\mathrm{z}$ & $-142.0 \mathrm{e} 3$ \\
\hline drivetrain design & 1 \\
\hline gear ratio & 81.491 \\
\hline gear configuration & 'epp' \\
\hline crane & False \\
\hline shaft angle & 5.0 \\
\hline shaft ratio & 0.10 \\
\hline $\mathrm{Np}$ & {$[3,1,1]$} \\
\hline ratio type & 'optimal' \\
\hline shaft type & 'normal' \\
\hline uptower transformer & False \\
\hline shrink disc mass & $333.3 *$ machine rating/1000.0 \\
\hline carrier mass & 250 \\
\hline mb1Type & 'SRB' \\
\hline mb2Type & 'TRB2' \\
\hline flange length & 0.285 \\
\hline overhang & 2.26 \\
\hline $\mathrm{L} \mathrm{rb}$ & 1.22 \\
\hline gearbox cm & 0.8 \\
\hline blade root diameter & 1.6 \\
\hline tower top diameter & 2.21 \\
\hline
\end{tabular}

Table 16. 750-kW Reference Fatigue Inputs

\begin{tabular}{|l|c|}
\hline cut in & 3 \\
\hline cut out & 25 \\
\hline Vrated & 16 \\
\hline weibull k & 2.2 \\
\hline weibull A & 9 \\
\hline T life & 20 \\
\hline IEC Class Letter & 'A' \\
\hline
\end{tabular}


Table 17. 1.5-MW Reference Inputs

\begin{tabular}{|l|c|}
\hline rotor diameter & 77 \\
\hline DrivetrainEfficiency & 0.95 \\
\hline machine rating & 1500 \\
\hline rotor torque & $1.5 *$ (machine rating * 1000 / DrivetrainEfficiency) / (rotor speed * (pi / 30) $)$ \\
\hline rotor thrust & $2.6204 \mathrm{e} 5$ \\
\hline rotor mass & 0.0 \\
\hline rotorRatedRPM & 16.18 \\
\hline rotor bending moment & $2.7795 \mathrm{e} 6$ \\
\hline rotor bending moment $\mathrm{x}$ & $8.4389 \mathrm{e} 5$ \\
\hline rotor bending moment $\mathrm{y}$ & $-2.6758 \mathrm{e} 6$ \\
\hline rotor bending moment $\mathrm{z}$ & $7.5222 \mathrm{e} 2$ \\
\hline rotor force $\mathrm{x}$ & $2.6204 \mathrm{e} 5$ \\
\hline rotor force y & $2.8026 \mathrm{e} 4$ \\
\hline rotor force $\mathrm{z}$ & $-3.4763 \mathrm{e} 5$ \\
\hline drivetrain design & 1 \\
\hline gear ratio & 78 \\
\hline gear configuration & 'epp' \\
\hline crane & False \\
\hline shaft angle & 5.0 \\
\hline shaft ratio & 0.10 \\
\hline Np & {$[3,1,1]$} \\
\hline ratio type & 'optimal' \\
\hline shaft type & 'normal' \\
\hline uptower transformer & True \\
\hline shrink disc mass & 2000.0 \\
\hline carrier mass & 'CARB' \\
\hline mb1Type & 'SRB' \\
\hline mb2Type & 0.285 \\
\hline flange length & 4 \\
\hline overhang & 1 \\
\hline L rb & 0.0 \\
\hline gearbox cm & 2.3 \\
\hline tower top diameter & \\
\hline & $333.3 *$ machine rating/1000.0 \\
\hline
\end{tabular}

Table 18. 1.5-MW Reference Fatigue Inputs

\begin{tabular}{|l|c|}
\hline blade number & 3 \\
\hline cut in & 3.5 \\
\hline cut out & 20 \\
\hline Vrated & 11.5 \\
\hline weibull k & 2.2 \\
\hline weibull A & 9 \\
\hline T life & 20 \\
\hline IEC Class Letter & 'B' \\
\hline
\end{tabular}


Table 19. 5-MW Reference Inputs

\begin{tabular}{|l|c|}
\hline rotor diameter & 126.0 \\
\hline machine rating & 5000.0 \\
\hline DrivetrainEfficiency & 0.95 \\
\hline rotor torque & $1.5 *$ (machine rating * 1000 / DrivetrainEfficiency) / (rotor speed * (pi / 30) $)$ \\
\hline rotor thrust & 599610.0 \\
\hline rotor mass & 0.0 \\
\hline rotorRatedRPM & 12.1 \\
\hline rotor bending moment & -16665000.0 \\
\hline rotor bending moment $\mathrm{x}$ & 330770 \\
\hline rotor bending moment $\mathrm{y}$ & -16665000.0 \\
\hline rotor bending moment $\mathrm{z}$ & 2896300.0 \\
\hline rotor force $\mathrm{x}$ & 599610.0 \\
\hline rotor force y & 186780.0 \\
\hline rotor force $\mathrm{z}$ & -842710.0 \\
\hline drivetrain design & 1 \\
\hline gear ratio & 96.76 \\
\hline gear configuration & 'eep' \\
\hline crane & True \\
\hline shaft angle & 5.0 \\
\hline shaft ratio & 0.10 \\
\hline Np & {$[3,3,1]$} \\
\hline ratio type & optimal' \\
\hline shaft type & 'normal' \\
\hline uptower transformer & False \\
\hline shrink disc mass & 8000.0 \\
\hline carrier mass & 'CARB' \\
\hline mb1Type & 'SRB' \\
\hline mb2Type & 0.5 \\
\hline flange length & 5.0 \\
\hline overhang & 0.1 \\
\hline gearbox cm & 1.5 \\
\hline hss length & 3.78 \\
\hline tower top diameter & 1.912 \\
\hline L rb & \\
\hline & $333.3 *$ machine rating/1000.0 \\
\hline
\end{tabular}

Table 20. 5-MW Reference Fatigue Inputs

\begin{tabular}{|l|c|}
\hline blade number & 3 \\
\hline cut in & 3 \\
\hline cut out & 25 \\
\hline Vrated & 11.4 \\
\hline weibull k & 2.2 \\
\hline weibull A & 9 \\
\hline T life & 20 \\
\hline IEC Class Letter & 'A' \\
\hline
\end{tabular}




\section{Appendix B: Shaft and Bearing Design with Parameterized Fatigue Spectrum (Optional)}

Fatigue analysis is a user-specified option in DriveSE that may take the form of user-defined lifetime loads spectra or, if data are not available, the parameterized loads spectrum defined in Appendix C. This model uses cyclically varying stochastic loads and deterministic loads from the rotor and component masses to size the main shaft and bearings. Using a simplified representation of cyclic and mean loads experienced by the rotor, forces and moments are resolved into stresses at the location of the main bearings. Beginning with the shaft diameters calculated using extreme loads, stresses and damage equivalent loads are calculated and diameters are increased until the resulting damage does not result in failure over a specified component lifetime. Additional inputs for this portion of the DriveSE model are shown in Table 21.

Table 21. Additional Inputs Required for Parameterized Fatigue Analysis

\begin{tabular}{ll}
\hline Input Variable & Units \\
\hline \hline Cut-In Wind Speed & $\mathrm{m} / \mathrm{s}$ \\
Rated Wind Speed & $\mathrm{m} / \mathrm{s}$ \\
Cut-Out Wind Speed & $\mathrm{m} / \mathrm{s}$ \\
IEC Class Letter & $\mathrm{A}, \mathrm{B}, \mathrm{C}$ \\
Availability (optional $)$ & $\%$ \\
Blade Number & - \\
Weibull Shape Parameter $\left(U_{10}\right.$ Wind Speed Distribution $)$ & - \\
Weibull Scale Parameter $\left(U_{10}\right.$ Wind Speed Distribution $)$ & $\mathrm{m} / \mathrm{s}$ \\
Design Life & $\mathrm{yrs}$ \\
Shaft Fatigue Exponent & - \\
\hline
\end{tabular}

It is important to note that the loads used in this section are taken from a comprehensive effort to define lifetime loads based on a limited number of inputs. This approach is outdated because the assumptions used in its derivation come from Danish studies performed more than a decade ago. In the absence of an updated parameterized loads definition, however, these loads have been scaled to fit a best estimation for loads experienced by current technology. In the future, an effort to better define lifetime load cycles from parameterized inputs would improve the accuracy of this analysis.

If users choose to implement this fatigue analysis, DriveSE also uses the loads generated in this section to select bearings that satisfy a calculated dynamic loads criterion. The design process for this sizing tool is illustrated in Figure 17. Bearing locations and component lengths are taken directly from the extreme loads model and assumed to be suitable. When calculating the total number of cycles experienced by the shaft during the design life of the turbine, it is assumed that the rated frequency, design life, and probability of operation (taken from Weibull parameters and cut-in/cut-out wind speed) can be multiplied to give an approximate lifetime number of shaft rotations. All documentation for these equations can be found in the loads documentation in Appendix C. Damage resulting from each load cycle is assumed to be linear, and wake effects from neighboring turbines are not considered in the calculation of aerodynamic rotor load cycles. After rotor loads are defined, the model resolves them into mean and alternating forces and moments at the location of the main bearings. 


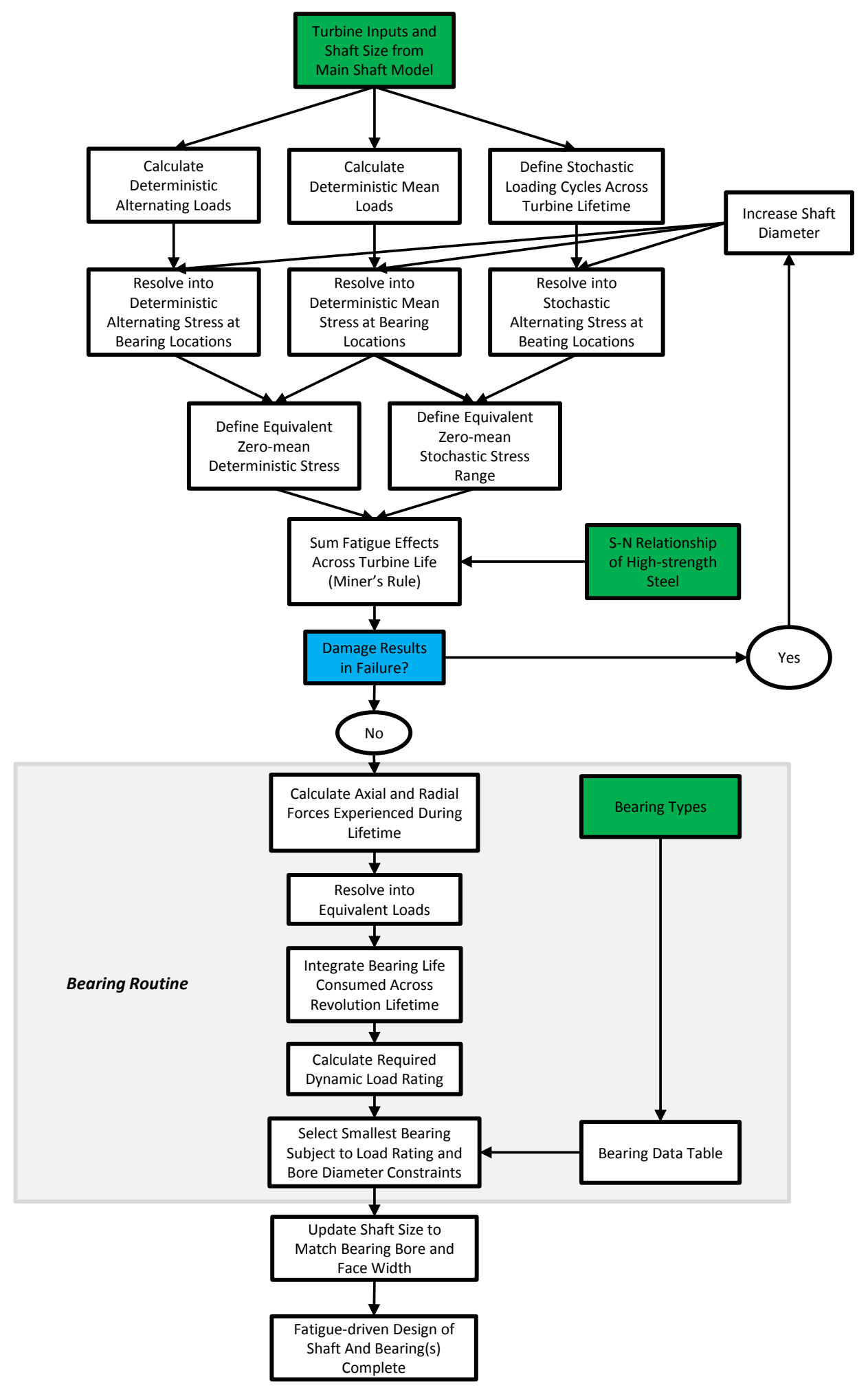

Figure 17. Flowchart of main shaft and bearing fatigue sizing tool 


\section{Stress Calculation of Three-Point Suspension}

Using the same component location assumptions as the extreme load sizing tool, the fatigue model calculates the stresses at the bottom of the shaft, above the single main bearing of a three-point drivetrain, where stresses are the highest magnitude. The stochastic stresses are found considering the bending, torsional shear, and normal stress, then they are combined into an equivalent stress value using the following equations:

$$
\begin{aligned}
& {\left[\begin{array}{lll}
\text { bending stress: } & \sigma_{\text {bend }}^{s t} & =-\sqrt{M_{y}^{s t}{ }^{2}+M_{z}^{s t}{ }^{2}} \frac{d_{m s}}{2 I} \\
\text { normal stress: } & \sigma_{\text {norm }}^{s t} & =-\frac{F_{x}^{s t} \cos \gamma}{A} \\
\text { torsional shear stress: } & \tau^{s t}=\frac{M_{x}^{s t} d_{m s}}{2 J}
\end{array}\right]} \\
& \qquad \sigma_{v}^{s t}=\sqrt{\sigma_{\text {bend }}^{s t}+\sigma_{\text {norm }}^{s t}+3 \tau^{s t}{ }^{2}}
\end{aligned}
$$

where the geometrical constants are calculated as

$$
\begin{aligned}
I & =\frac{\pi}{64}\left(d_{m s}^{4}-d_{m s, i}^{4}\right) \\
J & =\frac{\pi}{32}\left(d_{m s}^{4}-d_{m s, i}^{4}\right) \\
A & =\frac{\pi}{4}\left(d_{m s}^{2}-d_{m s, i}^{2}\right)
\end{aligned}
$$

Note that these calculations are performed for every stochastic force and moment in the array of values found in the Force$\mathrm{N}$ and Moment-N spectrum. They assume that maximum loads can all be combined to form a maximum stress instance and minimum loads can be combined to form a minimum stress instance. This approach is considered valid because all damage from the resulting stresses is ultimately summed into the same damage equivalent load figure.

The deterministic alternating stress on the main bearing is defined as the stress that occurs every time the main shaft makes one rotation. This stress takes into account the steady-state rotor loads and weights of the drivetrain components. In this case, the moment about the $y$-axis caused by the rotor overhang and mean $\mathrm{x}$-force is the source of the deterministic alternating stress at the main bearing:

$$
\sigma_{v}^{d e}=\frac{M_{y} d_{m s}}{2 I}=\frac{\left(W_{r} \cos \gamma L_{r b}-F_{x}^{m e a n} \sin \gamma L_{r b}\right) d_{m s}}{2 I}
$$

The mean stress is assumed to be constant and exists during both deterministic and stochastic stress cycles. This mean stress is a consequence of a mean torque value, the mean compressive force on the rotor, and the compressive effects of component weights. The following equations encompass the mean stress calculations used in this model:

$$
\begin{aligned}
& {\left[\begin{array}{ll}
\text { normal stress: } & \sigma_{\text {norm }}^{\text {mean }}=-\frac{F_{x}^{\text {mean }} \cos \gamma+\left(W_{r}+W_{m s}\right) \sin \gamma}{A} \\
\text { torsional shear stress: } & \tau^{\text {mean }}=\frac{M_{x}^{\text {mean }} d_{m s}}{2 J}
\end{array}\right]} \\
& \qquad \sigma_{v}^{\text {mean }}=\sqrt{\left(\sigma_{\text {norm }}^{\text {mean }}\right)^{2}+3\left(\tau^{\text {mean }}\right)^{2}}
\end{aligned}
$$

\section{Stress Calculation of Four-Point Suspension}

In the four-point suspension drivetrain, stress calculations are performed at both upwind and downwind bearings, and a suitable shaft diameter is selected at both locations. Because the load path up to the first main bearing is the same as that of the threepoint suspension configuration, stress calculations at the upwind bearing are the same as those in the three-point drivetrain, and follow Eqs. 7.1 and 7.5. Stresses at the downwind bearing are again separated into a single mean stress value, a distribution of stochastic stresses, and a deterministic alternating stress. Geometrical constants found in Eq. 7.3 are found from the diameters at the downwind bearing.

To determine the stresses at the downwind bearing, the model first calculates the forces at the upwind bearing. Again, it uses the same assumptions, force balances, and moment diagrams as those used in the extreme loading model. The forces on the upwind bearing caused by the stochastic forces and moments are: 


$$
\begin{aligned}
& F_{m b 1}^{y s t}=\frac{-M_{z}^{s t}}{L_{m b}} \\
& F_{m b 1}^{z s t}=\frac{M_{y}^{s t}}{L_{m b}} \\
& F_{m b 1}^{x} s t=0
\end{aligned}
$$

Stochastic moments at the downwind bearing are then calculated to be:

$$
\begin{array}{lll}
M_{m b 2}^{y}{ }^{s t} & =M_{y}^{s t}+F_{m b 1}^{z}{ }^{s t} L_{m b} & =0 \\
M_{m b 2}^{z}{ }^{s t} & =M_{z}^{s t}-F_{m b 1}^{y}{ }^{s t} L_{m b} & =0
\end{array}
$$

Because the moments at the downwind bearing caused by stochastic forces are zero, there is no bending stress. This leads to a stochastic stress at the downwind bearing because of the torsion on the shaft and the axial load:

$$
\begin{array}{cl}
\text { normal stress: } & \sigma_{\text {norm }}^{s t}=-\frac{F_{x}^{s t} \cos \gamma}{A} \\
\text { torsional shear stress: } & \tau^{s t}=\frac{M_{x}^{s t} d_{m s}}{2 J} \\
\sigma_{v}^{s t}=\overline{\left(\sigma_{\text {norm }}^{s t}\right)^{2}+3\left(\tau^{s t}\right)^{2}}
\end{array}
$$

Mean stresses are calculated in the same way, taking into account the deterministic axial and torsional stresses that are present at the downwind bearing. Because all axial forces on the shaft are assumed to be held by the downwind bearing, the mean stress at the second bearing is found to be:

$$
\begin{array}{ll}
\text { normal stress: } & \sigma_{\text {norm }}^{\text {mean }}=-\frac{F_{x}^{\text {mean }} \cos \gamma+\left(W_{r}+W_{m s}\right) \sin \gamma}{A} \\
\text { torsional shear stress: } & \tau^{\text {mean }}=\frac{M_{x}^{\text {mean }} d_{m s}}{2 J} \\
& \\
\sigma_{v}^{\text {mean }}= & \overline{\left(\sigma_{\text {norm }}^{\text {mean }}\right)^{2}+3\left(\tau^{\text {mean }}\right)^{2}}
\end{array}
$$

Deterministic alternating stresses that occur once every shaft rotation are found from the resulting bending stresses at the second bearing location. First, the deterministic forces at the upwind bearing are found to be:

$$
\begin{aligned}
F_{m b 1}^{z} d e & =\frac{-W_{r}\left(L_{m b}+L_{r b}\right)-\int_{0}^{L_{m b}} \omega_{m s}(x) d x\left(L_{m s}\right)+W_{g b}\left(L_{g b}\right)}{L_{m b}} \\
F_{m b 1}^{y} d e & =0
\end{aligned}
$$

It is assumed that $L_{a s}$ is half of the distance between the main bearings.

The moment at the downwind bearing caused by component weights and the reaction force calculated above is:

$$
\begin{array}{ll}
M_{m b 2}^{y} d e & -W_{r}\left(L_{r b}+L_{m b}\right)+F_{m b 1}^{z} d e-\int_{0}^{L_{m b}} \omega_{m s}(x) d x\left(L_{m s}\right)+W_{g b} L_{g b}=W_{g b} L_{g b} \\
M_{m b 2}^{z} \text { de } & =0
\end{array}
$$

From this, the deterministic alternating stress at the downwind bearing is found to be:

$$
\sigma_{v}^{d e}=\frac{M_{m b 2}^{y} d e}{2 I} d_{m s}
$$

\section{Damage Equivalent Load Summation and Sizing}

From the stresses at each bearing location, the model calculates the damage at each bearing and ensures that damage does not result in failure. Using the assumption that the mean stress is relatively constant during the operation of the turbine, the stochastic stresses and alternating deterministic stresses are converted into stresses with zero mean using a Goodman correction. Under Goodman, cyclic stresses with a nonzero mean contain a failure envelope described by: 


$$
\frac{\sigma_{a}}{\sigma_{v}}+\frac{\sigma_{m}}{S_{U T}}=1
$$

It is solved for the effective zero-mean alternating stress:

$$
\sigma_{e q}=\frac{\sigma_{a}}{1-\frac{\sigma_{m}}{S_{U T}}}
$$

After using this correction to convert both the stochastic and deterministic stresses, the resulting damage from each stress cycle is summed using the Palmgren-Miner linear damage rule. The general form of Miner's rule is shown in Eq. 7.18. Here, $n\left(S_{i}\right)$ is the number of cycles at a given stress amplitude that the material experiences, and $N\left(S_{i}\right)$ is the number of cycles at the stress amplitude that are needed to fail the material. The part does not fail as long as the accumulated damage, D, does not exceed 1:

$$
D=\sum_{i=1}^{n} \frac{n\left(S_{i}\right)}{N\left(S_{i}\right)}
$$

The value $N\left(S_{i}\right)$ is taken from the estimated S-N relationship of high-strength steel. According to Norton (2014), the following procedure is a reasonable estimate for creating an approximated S-N diagram of the main shaft. The high-strength steels used in main shaft manufacturing typically do not exhibit an endurance limit, so the S-N diagram of the material is defined to be similar to the one in Norton (2014).

With an ultimate strength of $700 \mathrm{MPa}$, we assume that the failure point at $10^{3}$ cycles, $S_{m}$, is $90 \%$ of the ultimate strength. The fatigue strength, $S_{f}$, of the material is calculated from the unadjusted fatigue strength $S_{e}^{\prime}$ and a variety of correction factors, as shown below (Norton (2014)). This value is taken to be the point at which the component will fail at $5 \times 10^{8}$ cycles.

\begin{tabular}{lll} 
Variable & Value & Remarks \\
\hline$S_{e}^{\prime}$ & $0.5 S_{U T}$ & \\
$C_{\text {size }}$ & 0.6 & Diameter $>250 \mathrm{~mm}$ \\
$C_{\text {surf }}$ & $4.51 S_{U T}^{-.265}$ & Machined Surface \\
$C_{\text {temp }}$ & 1.0 & Normal Operating Temperatures \\
$C_{\text {reliab }}$ & 0.814 & $99 \%$ Reliability \\
$C_{\text {envir }}$ & 1.0 & Enclosed Environment \\
$S_{f}$ & $=S_{e}^{\prime} C_{\text {size }} C_{\text {surf }} C_{\text {temp }} C_{\text {reliab }} C_{\text {envir }}$ &
\end{tabular}

The equation for the line that connects $S_{m}$ and $S_{f}$ can be defined by:

$$
S(N)=c 1 N^{c 2}
$$

where

$$
\begin{gathered}
c 2=\frac{\log \left(\frac{S_{m}}{S_{e}}\right)}{\log \left(N_{1}\right)-\log N_{2}} \\
c 1=\frac{S_{m}}{N_{1}^{c 2}}
\end{gathered}
$$

This creates an S-N curve with a fatigue exponent of approximately 0.117 , which reflects material data for several high-strength steel alloys.

The damage summation formula using Miner's rule then becomes:

$$
D=\sum_{i=1}^{n} \frac{n\left(S_{i}\right)}{\left(\frac{S_{i}}{c 1}\right)^{1 / c 2}}
$$


Summing the damage at these bearings results in a smooth function that defines the stochastic spectrum of stresses, and a single point defining the deterministic stress at a cycle number equal to the number of rotor rotations, $N_{r}$. Therefore, the final damage summation is defined by:

$$
D=\left[\int_{N_{i}}^{N_{f}} \frac{n\left(S_{i}\right)}{\left(\frac{S_{i}}{c 1}\right)^{1 / c 2}} d N\right]+\frac{N_{r}}{\frac{\sigma_{d e}}{c 2} 1 / c 2}<1.0
$$

As the flowchart in Figure 17 shows, the model iterates the stress and damage calculations at the bearing location(s), and increases the shaft diameter until the total damage caused by fatigue does not result in failure. After the fatigue-driven design of the shaft is complete, the model uses the forces and moments to calculate fatigue-driven design in the bearing routine.

If additional fatigue analysis is performed, bearings are selected to satisfy a calculated dynamic load rating in addition to the criteria listed above. Information about fatigue analysis and the steps used to arrive at this value can be found in Section 7 . The selected bearing geometry is then used to resize the shaft based on the selected facewidths.

Bearing fatigue is analyzed from the calculated axial and radial loads experienced by the main bearings across the life of the turbine. The model calculates a dynamic load rating from the summation of equivalent forces during each shaft rotation.

It is assumed that when integrating across turbine life, the domain of the integration is taken to be up to $N_{r}$ (found from $\frac{N_{f}}{B}$, where $\mathrm{B}$ is blade number), so summation across the domain representing each shaft rotation is achieved by dividing all values in the shaft $N$ vector by B. It is assumed that mean forces (both axial and radial) can be added to the stochastic force amplitudes to give a spectrum of dynamic loads with a maximum load value. These assumptions effectively convert the forces used in shaft analysis into ones that are usable in the bearing routine.

At the upwind bearing, the shaft model already defines the stochastic force distribution and the deterministic forces resulting from the component weights. In finding the force spectrum at this bearing, the radial and axial forces here are taken to be:

$$
F_{m b 1}^{r}=\sqrt{\left(F_{m b 1}^{z} s t+F_{m b 1}^{z} d e\right)^{2}+F_{m b 1}^{y} s t^{2}}
$$

and because the upwind bearing is assumed to carry all axial load,

$$
F_{m b 1}^{a}=F_{x}^{s t} \cos \gamma+\left(W_{r}+W_{m s}\right) \sin \gamma
$$

All stochastic forces are defined as a vector of values. Therefore, adding stochastic and deterministic forces gives the total bearing force during each revolution.

For a four-point drivetrain configuration, the force balance on the shaft system yields downwind bearing forces:

$$
\begin{aligned}
F_{m b 2}^{y} & =-F_{m b 2}^{y} d e=\frac{M_{z}^{s t}}{L_{m b}} \\
F_{m b 2}^{z} & =\left(F_{m b 2}^{z} d e\right)+F_{m b 2}^{z} s t \\
& =\left(W_{r}+W_{m s}-F_{m b 1}^{z} d e\right)+-F_{m b 1}^{z} s t \\
F_{m b 2}^{x}= & 0
\end{aligned}
$$

giving a radial load distribution of:

$$
F_{m b 2}^{r a}=\overline{F_{m b 2}^{y}+F_{m b 2}^{z}}
$$

and an axial force of:

$$
F_{m b 2}^{a x}=0
$$

For each bearing, the model then calculates an equivalent load, $P$, using the conditional equation:

$$
\begin{array}{ll}
P=F^{r}+Y_{1} F^{a} & , \frac{F^{a}}{F^{r}} \leq e \\
P=X F^{r}+Y_{2} F^{a} & , F^{a} F^{r}>e
\end{array}
$$


Table 22. Bearing Calculation Factors Used by Bearing Type

\begin{tabular}{lcccc}
\hline Bearing Type & $\mathrm{e}$ & $\mathrm{Y} 1$ & $\mathrm{Y} 2$ & $\mathrm{X}$ \\
\hline \hline CARB & 1.0 & 0 & 0 & 1.0 \\
SRB & 0.32 & 2.1 & 3.1 & 0.67 \\
TRB1 & 0.37 & 0 & 1.6 & 0.4 \\
TRB2 & 0.4 & 2.5 & 1.75 & 0.4 \\
CRB & 0.2 & 0 & 0.6 & 0.92 \\
RB & 0.4 & 1.6 & 2.15 & 0.75 \\
\hline
\end{tabular}

where the variables $Y_{1}, Y_{2}, X$, and $e$ are the calculation factors specific to each bearing type. Table 22 gives the approximate calculation factors for each bearing type, found from characteristic values in the bearing catalogs (SKF 2014).

As an added requirement on cylindrical roller bearings, the ratio of axial to radial loads may not exceed 0.5, as specified by (SKF 2014). In addition, because CARBs are not designed to carry axial load, an error is returned if significant axial loads are present. $P=F^{r a}$ for all CARBs.

The bearing life calculation uses International Organization for Standardization (ISO) 281:2007-02. The model then uses the calculated equivalent load and the bore diameter of the bearing to determine the mass of the bearing according to the data presented in Section 2.3. 


\section{Appendix C: Parameterized Fatigue Loads Definition}

Fatigue analysis of the main shaft and bearings requires an approximate knowledge of all stresses experienced by these components across the lifetime of a turbine. Because that designers do not always know the full spectrum of loads that may be input into the system, parameterized loads spectra are used to approximate all stochastic loads from turbine operation. Deterministic loads caused by component weights are also calculated, and the damage from all predicted loads is added to one overall fatigue damage figure.

It is important to note that the loads used in this section are taken from a comprehensive effort to define lifetime loads based on a limited number of inputs. This approach is outdated because the assumptions used in its derivation come from Danish studies performed more than a decade ago. In the absence of an updated parameterized loads definition, however, they have been scaled to fit a best estimation for loads experienced by current technology. In the future, an effort to better define lifetime load cycles from parameterized inputs would improve the accuracy of this analysis. The implementation of the DS472 standard (Danish Standards Foundation 1992) at this point should be considered a temporary placeholder until a more up-to-date model can be generated. Its value lies in the ability to provide an input/output sensitivity between rotor stochastic loads and shaft loading. This serves as a crude approximation for a full and much more expensive loads analysis.

Table 23 details the three types of stresses that are extrapolated from the loads on the main shaft and bearings. The weight of the rotor and drivetrain components creates a cyclically-varying stress on the shaft that occurs once every rotation, referred to here as deterministic alternating. Stochastic loads are defined with their own distribution of cycle counts, ranging from a low number of cycles with large-magnitude loads to a high number of small-magnitude loads. Finally, a mean compressive stress is expected to exist on the shaft, caused by axial forces that are assumed to be constant during the operation of the turbine.

Table 23. Description of Three Stress Sources Considered in the Main Shaft Fatigue Analysis

\begin{tabular}{lll}
\hline Stress Type & Occurrence Number & Description \\
\hline \hline Stochastic & $\begin{array}{l}\text { Defined by load } \\
\text { distribution }\end{array}$ & $\begin{array}{l}\text { Caused by the approximated stochastic rotor loads experienced } \\
\text { by a turbine throughout its design life. Defines a cyclic axial } \\
\text { force, } F^{x} ; \text { torque, } M^{x} ; \text { and moments, } M^{y}, M^{z}\end{array}$ \\
\hline $\begin{array}{l}\text { Deterministic } \\
\text { Alternating }\end{array}$ & $\begin{array}{l}\text { Equal to total rotor } \\
\text { rotations }\end{array}$ & $\begin{array}{l}\text { Caused by component weights and mean loads. Assumed to be } \\
\text { perfectly sinusoidal on a stress element located on the rotating } \\
\text { shaft }\end{array}$ \\
\hline Mean & Constant, noncyclic & $\begin{array}{l}\text { Axial and torsional stress caused by rotor mean force, compo- } \\
\text { nent weights, and mean torque. Assumed to be present during } \\
\text { all deterministic and stochastic cycles }\end{array}$ \\
\hline
\end{tabular}

The aerodynamic stochastic loads spectrum originates from (Danish Standards Foundation 1992). This standard gives an idealized load distribution expressed in terms of the additional input parameters shown in Table 21, as well as inputs such as rotor diameter and rated rpm. DS472 is based on the aerodynamic line load on the blades, $p_{0}[\mathrm{~N} / \mathrm{m}]$, and is calculated in Eq. 8.1. The load distribution along a single blade is then represented as a triangular line load with a value of $p_{0}$ at the blade tip and 0 at the hub. This value comes into play in subsquent calculations of aerodynamic loading on the rotor:

$$
\begin{gathered}
p_{o}=\frac{1}{2} \rho_{a} W^{2} c C_{L} \\
W^{2}=\left(\frac{4 \pi}{3} f_{r} R\right)^{2}+V_{0}^{2}
\end{gathered}
$$

where 


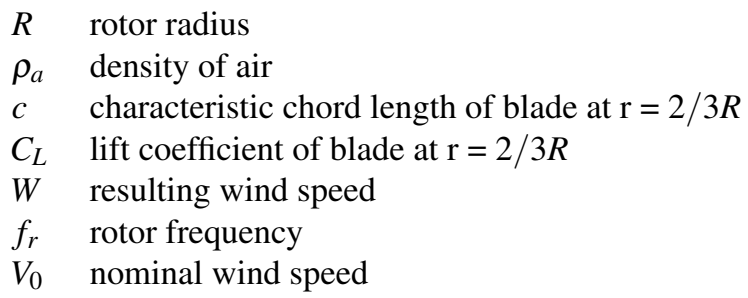

To limit the number of inputs needed for the fatigue model, a generalized chord length was calculated from the optimization equation found in (Gundtoft 2009), shown in Eq. 8.3:

$$
c(r)=\frac{16 \pi R}{9 B C_{L}} \frac{1}{X \frac{X^{2} \frac{r}{R}^{2}+\frac{4}{9}}{X^{2}}}
$$

where

$\begin{array}{ll}X & \text { tip-speed ratio } \\ B & \text { blade number } \\ C_{L} & \text { coefficient of lift at } \mathrm{r}=2 / 3 R\end{array}$

Combining Eqs. 8.1, 8.2, and 8.3 results in a simplified equation for the aerodynamic line load on the blades:

$$
p_{o}=\frac{4}{3} \rho_{a} \quad \frac{4 \pi}{3}^{2}+V_{0}^{2} * \frac{\pi * R}{B X \sqrt{X^{2}+1}}
$$

To define the total number of load cycles experienced throughout the turbine life, the probability of operation is approximated from the cut-in and cut-out wind speeds and the $U_{10}$ Weibull parameters. For the remainder of this report, the Weibull parameters $k$ and $A$ are defined as the shape and scale of the wind speed probability distribution. This probability is then multiplied by the number of rotor rotations during the design life, if the turbine were operating at rated speed the entire time. The following equation defines $N_{F}$, the maximum number of loads experienced from a load frequency, $f_{c}$. To evaluate pressure from the blades of a turbine, $f_{c}$ is taken to be $f_{\text {rated }} * B$, as recommended by the standard. This effectively defines the total number of possible load cycles as $3 \times N_{\text {rotor }}$ for a three-bladed turbine and $2 \times N_{\text {rotor }}$ for a two-bladed turbine.

where

$$
N_{f}=f_{C} T_{L}\left(\exp \left(-\left(V_{\min } / A_{w}\right)^{k_{w}}\right)-\exp \left(-V_{\max } / A_{w}\right)^{k_{w}}\right)
$$

$\begin{array}{ll}f_{c} & \text { characteristic load frequency } \\ T_{L} & \text { turbine life } \\ V_{\min } & \text { cut-in wind speed } \\ V_{\max } & \text { cut-out wind speed } \\ k_{w} & \text { Weibull shape parameter } \\ A_{w} & \text { Weibull scale parameter }\end{array}$

To define a stochastic cyclic load, a standardized, nondimensional load range $F \Delta^{*}$ is defined as a representation of all load ranges up to this maximum number of cycles. Under DS472, the probability distribution is defined such that $F \Delta^{*}$ is the load range that is exceeded $\mathrm{N}$ times and is found using the following equation:

$$
F \Delta^{*}(N)=\beta\left(\log _{10}\left(N_{f}\right)-\log _{10}(N)\right)+0.18
$$

This creates a definition of the standard load range distribution that shows a low occurrence of high-magnitude loads and a high occurrence of lower magnitude loads. This nondimensional load distribution is used to form the shape of the rotor force and 
moment distribution for fatigue analysis. Figure 18 shows an example of this distribution shape applied to the rotor force and moment distributions on a $750-\mathrm{kW}$ rotor.

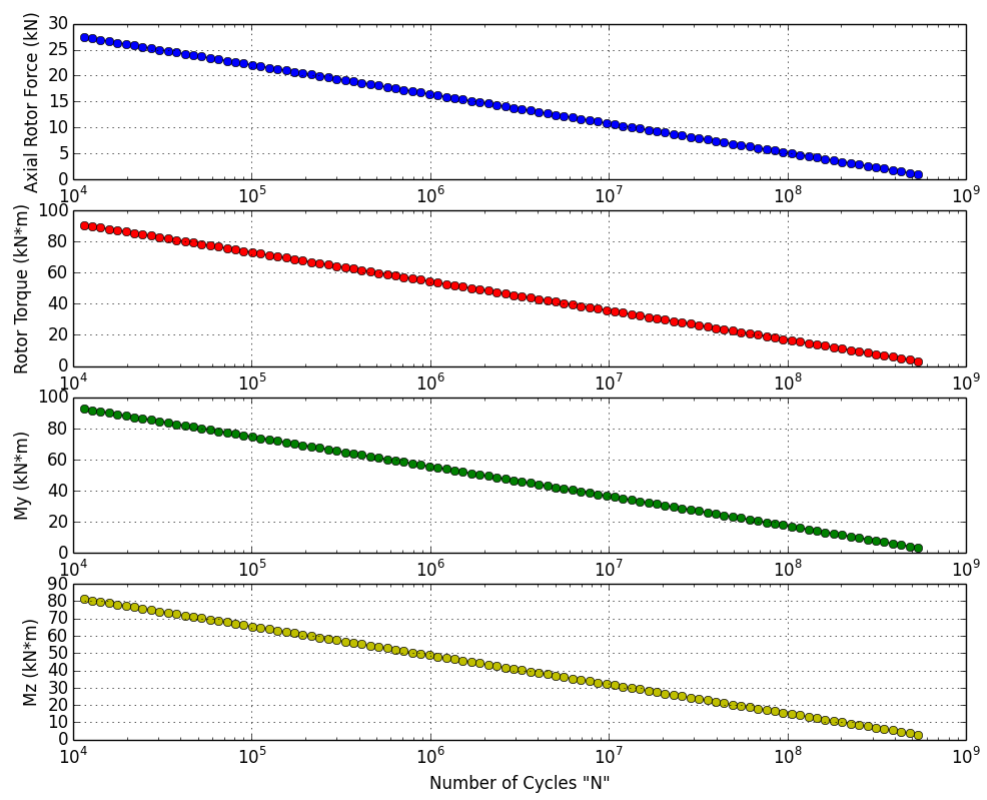

Figure 18. Force and moment spectra defined by DS472 using inputs from a 750-kW rotor

The variable $\beta$ is a scaling variable that takes into account the turbulence intensity, $I_{T}$, and the 10 -min wind speed shape parameter, $A_{w} . \beta$ is calculated in Eq. 8.7. Assuming no adjustment for neighboring turbines, the value for turbulence intensity is found from the user-input IEC class according to Table 24 (IEC 2005).

$$
\beta=0.11 k_{\beta}\left(I_{T}+0.1\right)\left(A_{w}+4.4\right)
$$

Table 24. Relationship Between IEC Class and Turbulence Intensity Factor

\begin{tabular}{ll}
\hline IEC Class & $I_{T}$ \\
\hline \hline $\mathrm{A}$ & 0.16 \\
$\mathrm{~B}$ & 0.14 \\
$\mathrm{C}$ & 0.12 \\
\hline
\end{tabular}

In accordance with DS472, the value of $k_{\beta}$ is taken to be 2.5 . In addition to scaling the variable $\beta, k_{\beta}$ also appears as an added condition to the standardized loads range found in Eq. 8.6. The condition suggested by DS472 is that the value of $F_{\Delta}^{*}$ must not exceed $2 k_{\beta}$. This effectively truncates the extreme values of the nondimensional loads range at approximately $10^{3}$ to $10^{4}$ load counts.

With $F_{\Delta}^{*}$ and $p_{o}$ defined, the stochastic load ranges from the rotor can be calculated according to the relationships in Eq. 8.8:

$$
\left[\begin{array}{rl}
F_{x}^{\text {stoch }} & =0.5 F_{\Delta}^{*}(N) p_{0} R C_{F_{x}} \\
M_{x}^{\text {stoch }} & =0.45 F_{\Delta}^{*}(N) p_{0} R^{2} C_{M_{x}} \\
M_{y}^{\text {stoch }} & =0.33 F_{\Delta}^{*}(N) k_{r} p_{0} R^{2} C_{M_{y}} \\
M_{z}^{\text {stoch }} & =0.33 F_{\Delta}^{*}(N) k_{r} p_{0} R^{2} C_{M_{z}}
\end{array}\right.
$$


The amplification factor, $k_{r}$, depends on the ratio of rotor resonant frequency $\left(n_{r}\right)$ to the lowest resonant frequency of the associated oscillation form $\left(n_{o}\right)$; for $M_{y}$ and $M_{z}, n_{o}=n_{r}$, leading to an amplification factor value of 0.8 .

The factors $C_{F x}, C_{M x}, C_{M y}$, and $C_{M z}$ are adjustments to the original spectra defined by DS472 to account for technology changes since its publication. These factors were determined using available industry data on lifetime fatigue loads, which are unfortunately proprietary in nature.

$$
\left[\begin{array}{ll}
C_{F x} & =0.365 \times \log \left(D_{r}\right)-1.074 \\
C_{M x} & =0.0799 \times \log \left(D_{r}\right)-0.2577 \\
C_{M y} & =0.172 \times \log \left(D_{r}\right)-0.5943 \\
C_{M z} & =0.1659 \times \log \left(D_{r}\right)-0.5795
\end{array}\right.
$$

An example of the output load ranges is shown in Figure 18. Note that each of these points represents the range of a cyclic load occurring a specified number of times. Because calculations of stress for the purposes of damage equivalent loads require stress amplitudes to be used, the model halves these values in subsequent calculations. This distribution is treated as a histogram of loads experienced across the turbine life. A plot of these distributions, much like those found in Figure 18, is also known as an exceedance plot.

In addition to stochastic alternating loads, several deterministic rotor loads are considered for the purpose of fatigue analysis. For example, rotor weight is applied as a deterministic force in the negative z-direction:

$$
F_{z}^{\text {determ }}=-W_{r}
$$

From the definition of the line load $p_{0}$, the mean rotor force in the $\mathrm{x}$-direction is found to be:

$$
F_{x}^{\text {determ }}=\frac{1}{2} p_{0} R B
$$

The mean rotor torque during operation is defined as:

$$
M_{x}^{\text {determ }}=\frac{P}{\omega \eta_{d}}
$$

where $\mathrm{P}$ is the power rating of the turbine, $\omega$ is the rotational velocity of the rotor and drivetrain, and $\eta_{d}$ is the drivetrain efficiency (DS4 1992). These mean loads are applied to the fatigue model as ever-present means to the deterministic and stochastic loads.

This loads definition is currently used in the main shaft and bearing fatigue sizing models. Further work may be done to use the stochastic loads spectra in hub, gearbox, and bedplate sizing as well. 


\section{Appendix D: Simplified Four-Point Suspension Main Shaft Model (Optional)}

An alternative and simple main shaft design is available that assumes that the largest stresses will occur immediately upwind of the main bearing and sizes the main shaft to avoid yielding at this point by an acceptable safety margin. This model sizes the main shaft solely based on ultimate strength considerations and does not consider available bearing bore diameters or employ a taper as the three- and four-point main shaft models do. The user can specify a hollow main shaft by providing the inner and outer diameter ratio.

The model calculates the maximum principal stress, $\sigma_{1}$, based on a combined torque and bending moment loading situation given by the following formula:

$$
\sigma_{1}=\frac{\sigma_{\text {bend }}}{2}+\overline{\left(\frac{\sigma_{\text {bend }}}{2}\right)^{2}+\tau^{2}}
$$

where $\sigma_{\text {bend }}$ is the normal stress resulting from bending and $\tau$ is the shear stress taken at the outer surface of the main shaft where stresses are highest. The normal stress is given by:

$$
\sigma=-\frac{M y}{I}
$$

where $M$ is the bending moment with the convention of positive moments causing compressive stresses above the neutral axis and tensile stresses below the neutral axis, $y$ is the distance above the neutral axis, and $I$ is the second moment of area.

The shear stress because of the applied torque is calculated by:

$$
\tau=\frac{T c}{J}
$$

where $c$ is the radius, and $J$ is the second polar moment of area. For a hollow shaft, which the main shaft is expected to be,

$$
J=\frac{\pi}{32}\left(d_{m s}^{4}-d_{m s, i}^{4}\right)
$$

where $d_{o}$ is the outer shaft diameter, and $d_{i}$ is the inner shaft diameter. Additionally, for a hollow shaft, $I$, is given by:

$$
I=\frac{\pi}{64}\left(d_{m s}^{4}-d_{m s, i}^{4}\right)
$$

Putting Eq. 9.3 and 9.2 into Eq. 9.1 gives:

$$
\sigma_{1}=\frac{M d_{m s}}{4 I}+\sqrt{\frac{M d_{m s}}{4 I}+{\frac{T d_{m s}}{2 J}}^{2}}
$$

Letting $r_{1}=\frac{d_{i}}{d_{o}}$ and substituting Eqs. 9.4 and 9.5 into Eq. 9.6 gives:

$$
\sigma_{1}=\frac{16 M}{\pi 1-r_{1}^{4} d_{m s}^{3}}+\sqrt{\frac{256 M^{2} d_{m s}^{2}}{\pi^{2} d_{m s}^{4}-r_{1}^{4} d_{m s}^{4}{ }^{2}}+\frac{256 T^{2} d_{m s}^{2}}{\pi^{2} d_{m s}^{4}-r_{1}^{4} d_{m s}^{4}{ }^{2}}}
$$

Simplifying further obtains:

$$
\sigma_{1}=\frac{16}{\pi 1-r_{1}^{4} d_{m s}^{3}}\left(M+\sqrt{M^{2}+T^{2}}\right)
$$

Rearranging to solve for $d_{m s}$ : 


$$
d_{m s}=\left(\frac{16}{\pi 1-r_{1}^{4} \sigma_{1}}\left(M+\overline{M^{2}+T^{2}}\right)\right)^{\frac{1}{3}}
$$

Equation 9.9 gives the minimum main shaft outer diameter needed to withstand a factored bending moment and factored torque.

Note that the simple shaft model has not been updated to include improved CM analysis and may be updated in further work, if there is a demand for its use. 


\section{Appendix E: Additional Bearing Load Rating and Mass Curves Used in DriveSE}

In the case of SRBs, load ratings follow four load curves, as shown in Figure 19. The equation defining the curve fit of the entire data set is again taken to be the cutoff for high-load and low-load selection. This division essentially divides the data into two sets of two load curves. Figure 20 shows the two curves and the equations used in the bearing model.

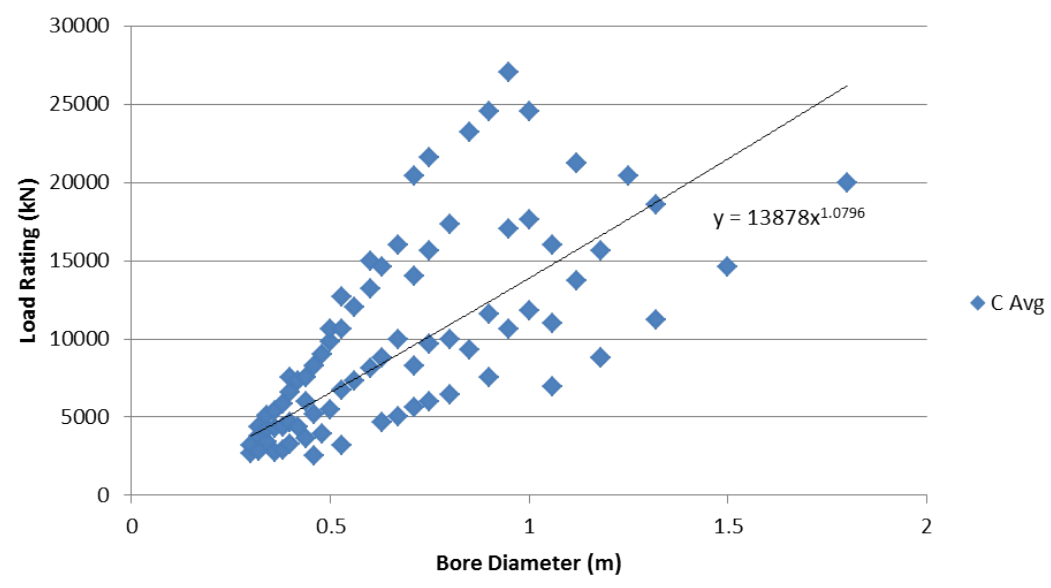

Figure 19. Dynamic load ratings for SRBs
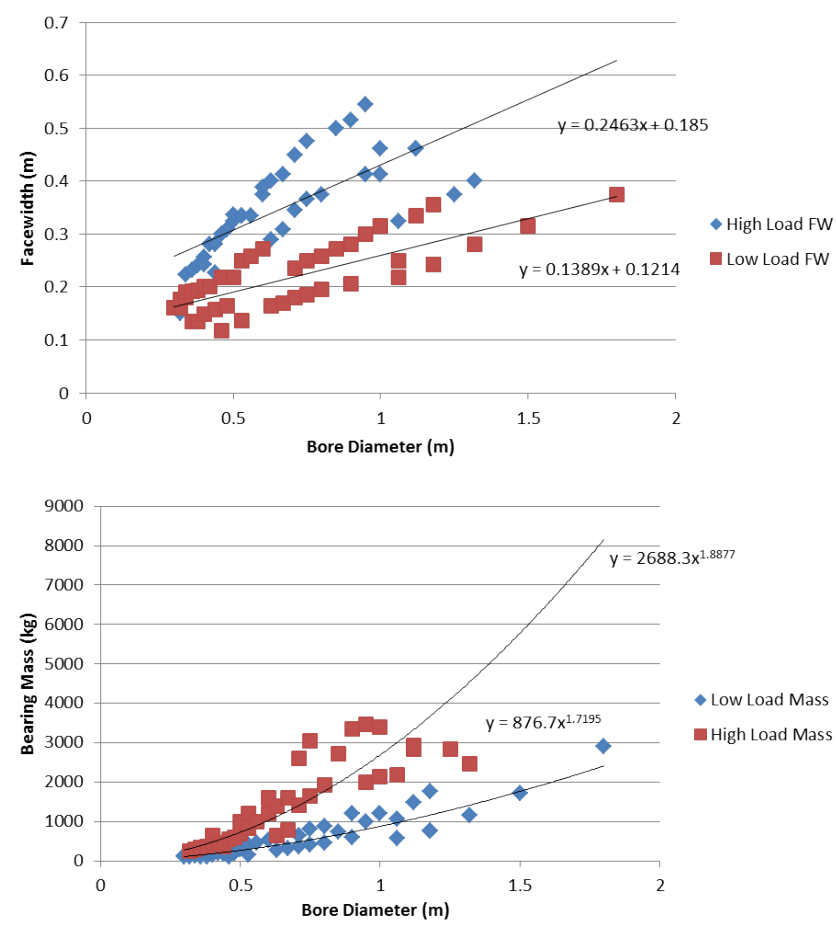

Figure 20. SRB mass and facewidth interpolation 
A similar approach is used to define the mass and dimensions of the remaining bearings. Curves with the equations used in these bearing definitions are shown in Figures 21 through 28.

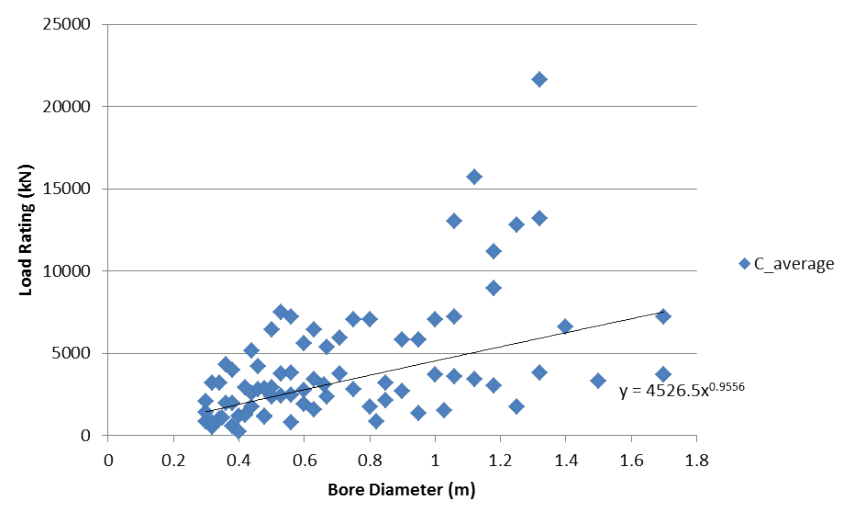

Figure 21. Dynamic load ratings for CRBs
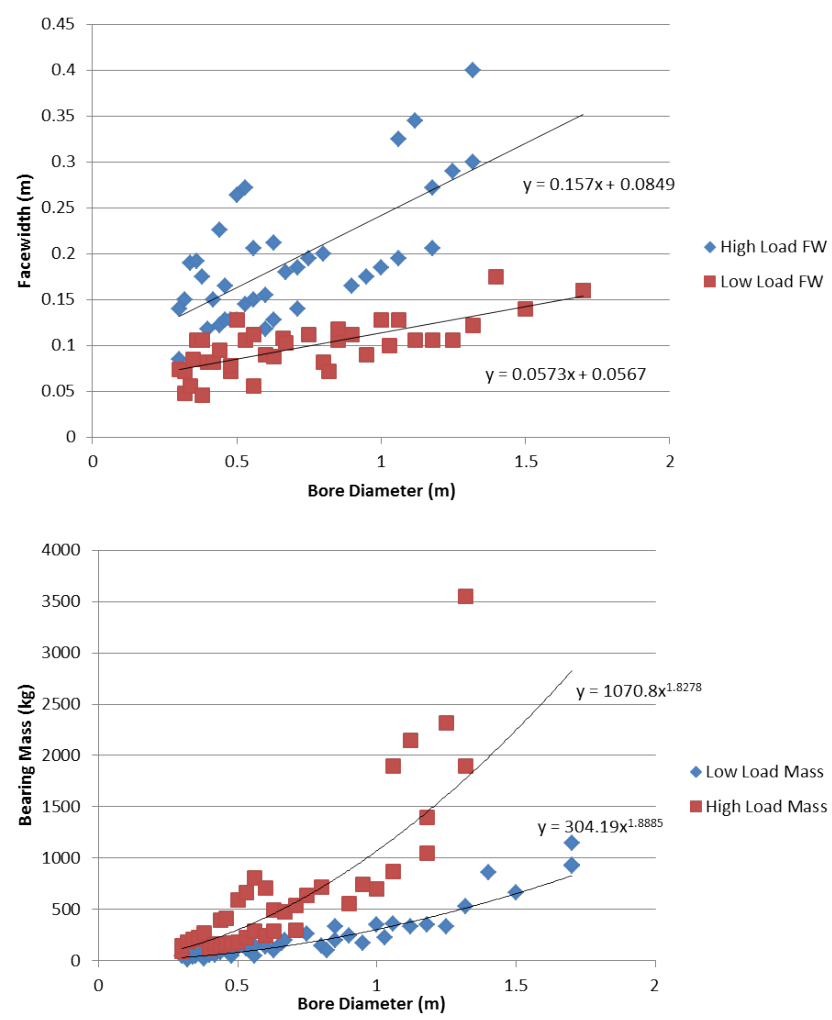

Figure 22. CRB mass and facewidth interpolation 


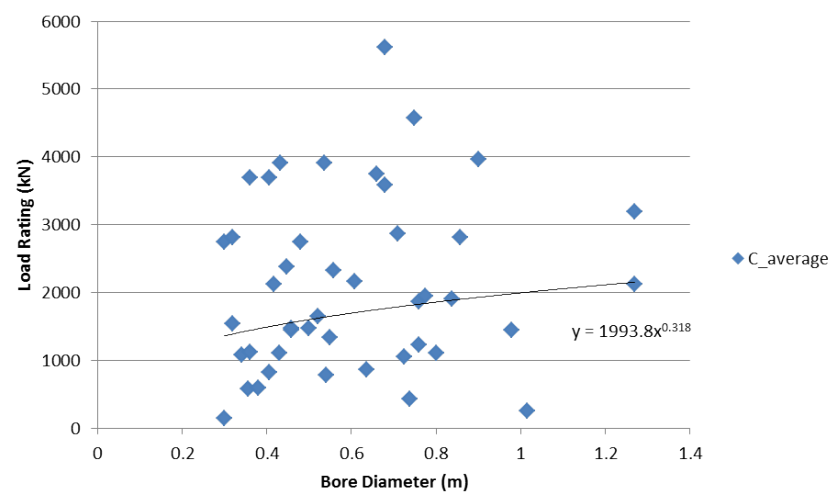

Figure 23. Dynamic load ratings for TRBs1
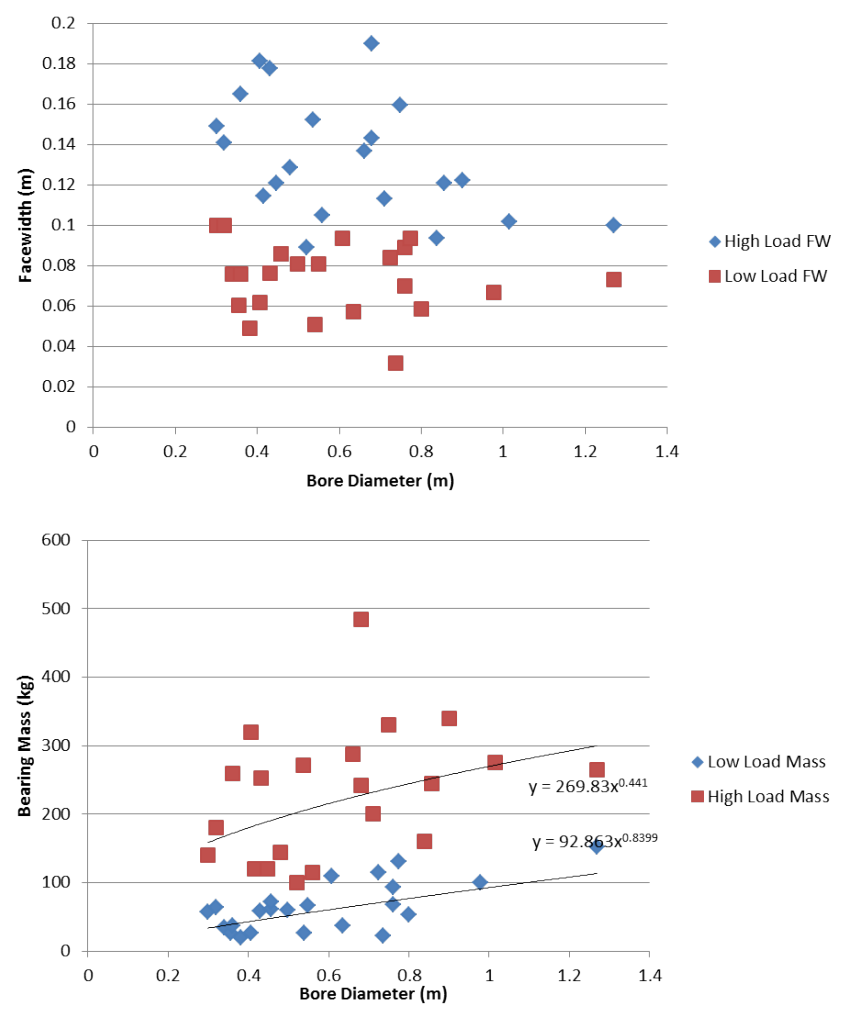

Figure 24. TRB1 mass and facewidth interpolation

Note that because of a significant amount of scatter in the facewidth data of TRB1, the facewidth of these bearings are taken to be a constant value, independent of bore diameter. These constant values are taken from an average of each distribution. For high-load applications, a facewidth of $133.5 \mathrm{~mm}$ is defined, and for low-load applications, a facewidth of $74.0 \mathrm{~mm}$ is defined. 


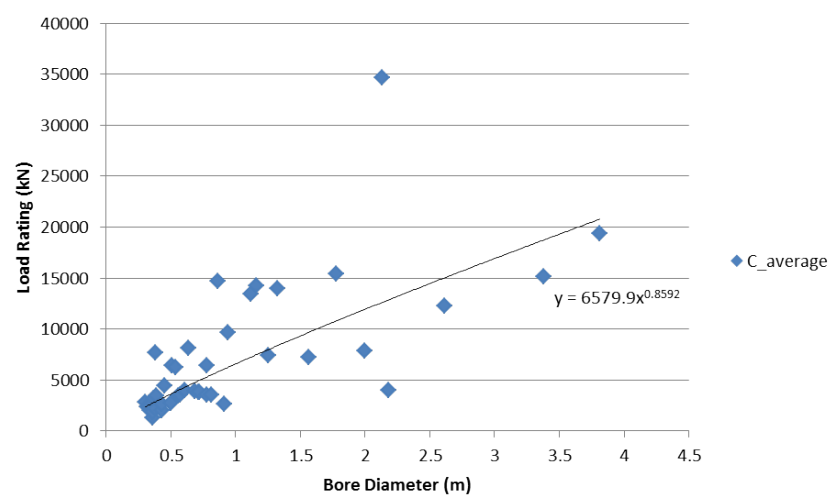

Figure 25. Dynamic load ratings for TRBs2
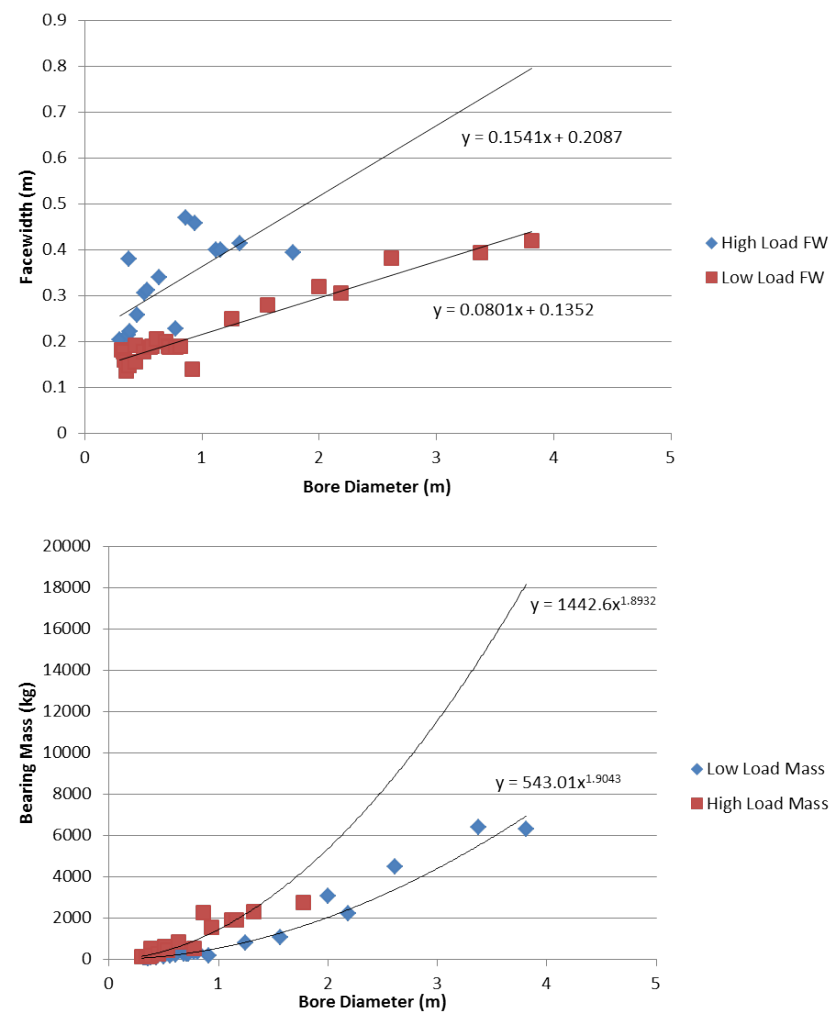

Figure 26. TRB2 mass and facewidth interpolation 


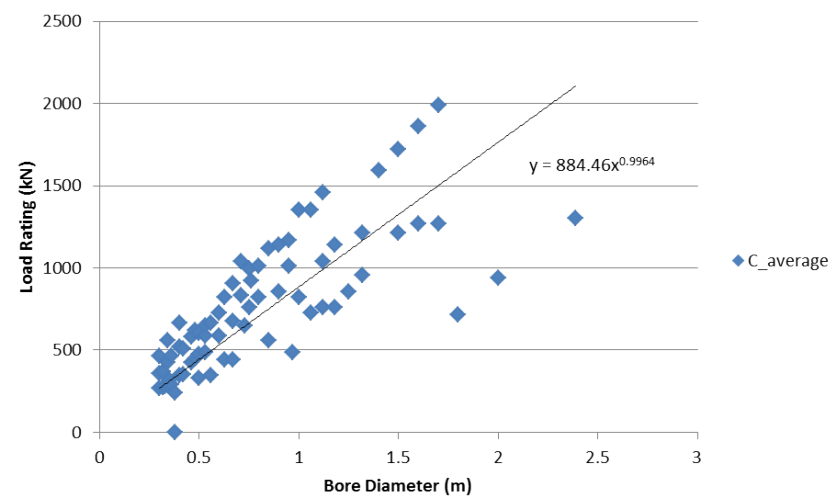

Figure 27. Dynamic load ratings for RBs
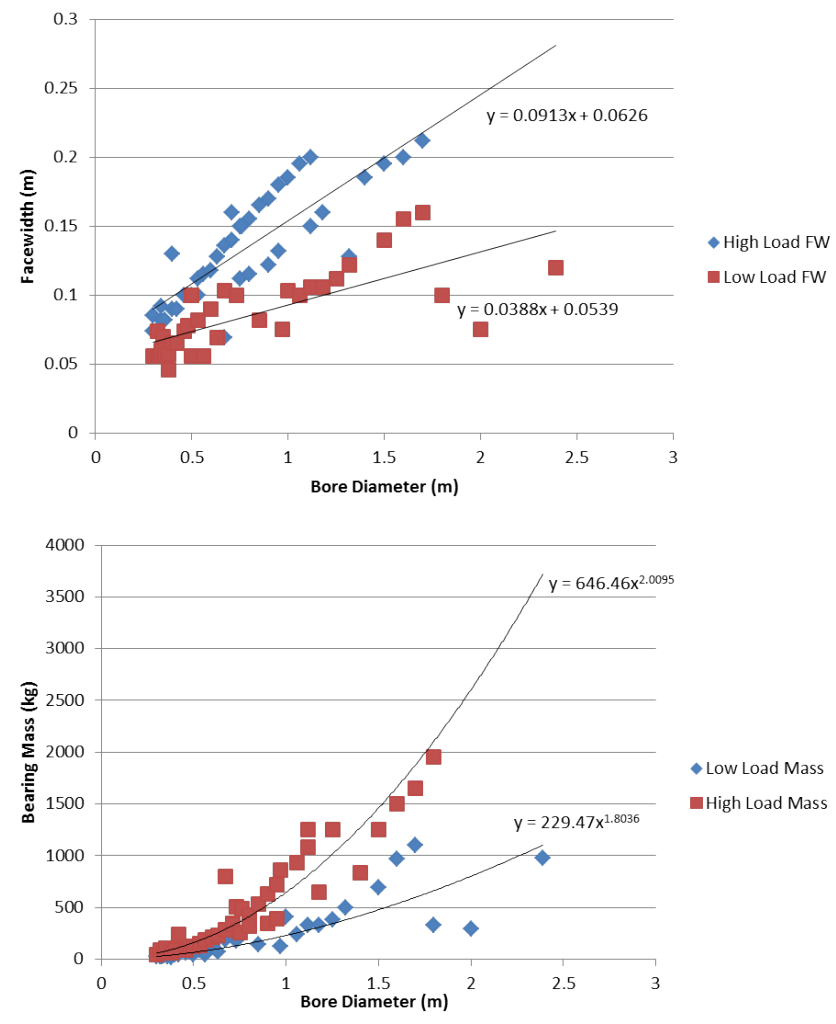

Figure 28. RB mass and facewidth interpolation 